\title{
IMPROVED THERMOPLASTIC COMPOSITE BY ALIGNMENT OF VAPOR GROWN CARBON FIBER
}

\author{
A Dissertation Presented to \\ The Faculty of the \\ Fritz J. and Dolores H. Russ \\ College of Engineering and Technology \\ Ohio University \\ In Partial Fulfillment \\ of the Requirement for the Degree \\ Doctor of Philosophy
}

by

Rex J. Kuriger

November, 2000
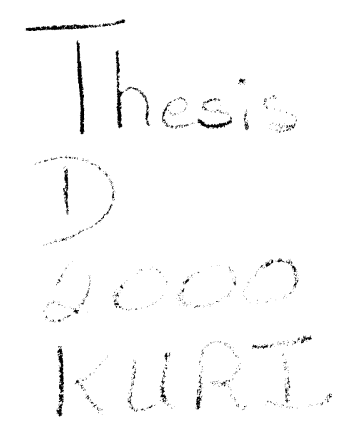

\section{OHIO UNIVERSITY}

I IRRARY 


\section{ACKNOWLEDGMENTS}

The author would like to offer special thanks to the supportive professors in the Russ College of Engineering and Technology, particularly my advisor, Dr. M. Khairul Alam for his advising, academic, and financial support. I have gained a lot, both technically and otherwise, through my interactions with him over the last three years. I also thank the members of my dissertation committee, Dr. Charles Parks, Dr. Daniel Gulino, Dr. Glenn Hazen, and Dr. Peter Klein for their valuable insight. Thanks to the Dr. Ken Sampson for the use of the extruder and the Industrial Technology Department for the use of their plastics lab and equipment.

I also wish to thank the staff of Applied Sciences, Inc., especially Dr. Ronald Jacobsen for his valuable contributions and assistance in the discussion and interpretation of many of the results. Special thanks to Dr. Dave Anderson of the University of Dayton Research Institution for performing the $\mathrm{x}$-ray diffraction studies. I would aiso like to acknowledge Dr. Gary Tibbetts of General Motors Research and Development Center for his many helpful suggestions.

This work was supported in part by the National Science Foundation, Grant No. DMI-9960480 and by the Ohio University Office of Research. This work was aiso partially supported by the U. S. Air Force under Contract number F33615-95-D-5029 and Air Force CRDA \#95-WL-004-01 with Applied Sciences, Inc. 


\section{TABLE OF CONTENTS}

\section{Page}

Acknowledgments

i

Table of Contents

ii

List of Tables

$\mathrm{v}$

List of Figures

vi

Chapter 1 Introduction

1.1 Background ........................................................................... i

1.2 Scope of Dissertation ................................................................ ;

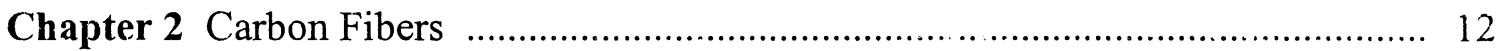

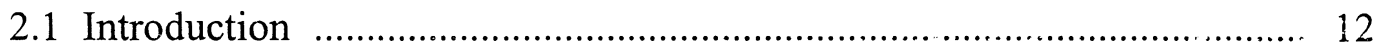

2.2 PAN and Pitch Derived Carbon Fibers .......................................... 14

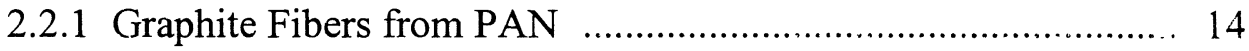

2.2.2 Graphite Fibers from Pitch ........................................... 15

2.3 Vapor Grown Carbon Fibers ...................................................... 18

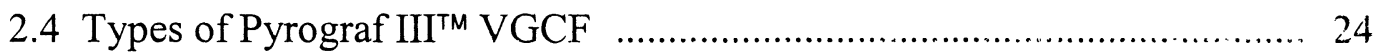

Chapter 3 Extrusion Conditions and Properties of VGCF Reinforced Polypropylene

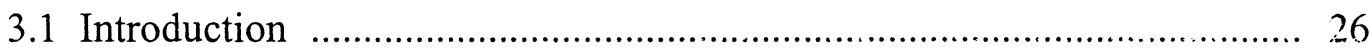

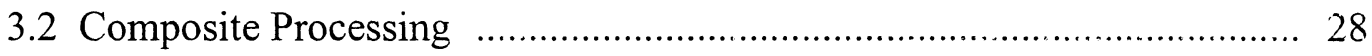


3.3 Variation of Extrusion Conditions on Flow Rate and Pressure …........... 30

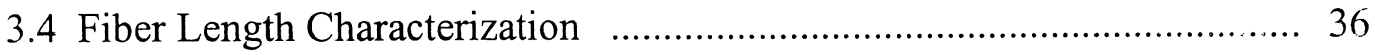

3.5 Mechanical Properties .................................................................... 39

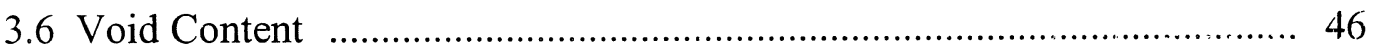

3.7 Effect of VGCF on Polymer Flow …………….................................... 49

Chapter 4 Processing and Characterization of Aligned VGCF Reinforced Polypropylene

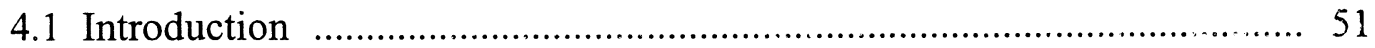

4.2 X-ray Diffraction .......................................................................... 51

4.3 Flow-Induced Alignment of VGCF ……........................................... 58

Chapter 5 Strength Prediction of Partially Aligned Short Fiber Reinforced Composites $6 ?$

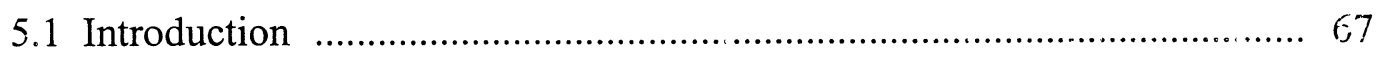

5.2 Strength Prediction of Randomly Oriented Fibers ……........................... 71

5.3 Strength Prediction of Partially Aligned Fibers ...................................... 74

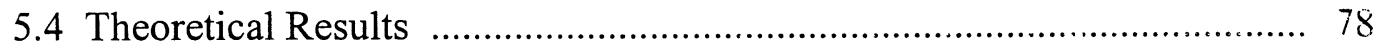

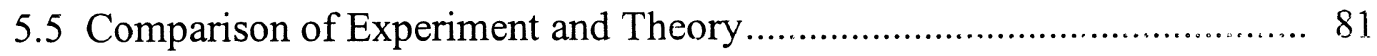

Chapter 6 Transport Properties of Vapor Grown Carbon Fiber Composites .......... 83



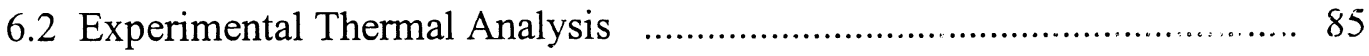

6.3 Theoretical Thermal Analysis ........................................................... 90 


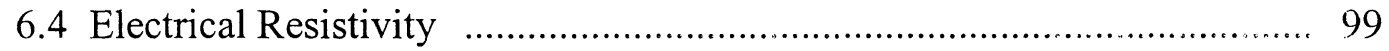

Chapter 7 Conclusions and Recommendations for Future Studies ....................... 103

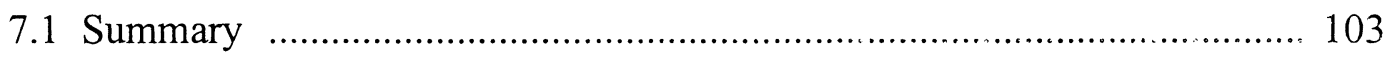

7.2 Recommendations for Future Studies f..................................... 107

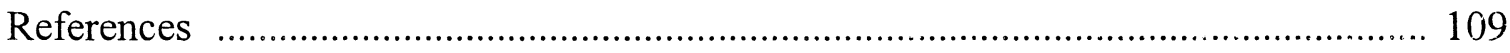

Abstract 


\section{LIST OF TABLES}

Page

2.1. Properties of the Pyrograf $\mathrm{I}^{\mathrm{TM}}$ fiber (ASI, 1999) ....................................... 24

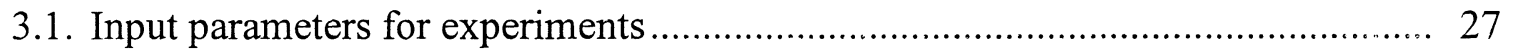

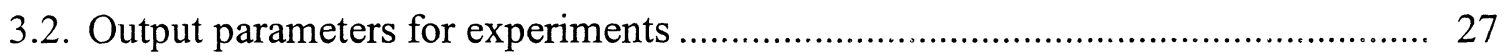

4.1. Fiber orientation along the preferred axis of the composite materials ............... 66

5.1. Fiber orientation $(\mathrm{Z} / 2)$ along the preferred axis of the composites samples at various fiber volume percentages ............................................................. 75

6.1. Table 6.1. Thermal properties of PR-19-HT Pyrograf III ${ }^{\mathrm{TM}}$ fiber reinforced polypropylene at various fiber volume fractions ........................................ 90

6.2. Electrical resistivity of PR-19-HT Pyrograf III ${ }^{T M}$ fiber reinforced polypropylene at various fiber volume fractions ............................................................ 101 


\section{LIST OF FIGURES}

Page

2.1. Comparison of PAN and pitch processes for carbon fiber production

(Bunsell, 1988)

2.2. Schematic illustration of expected applications of VGCF .......................... 18

2.3. Process Flow Diagram for VGCF ............................................................ 20

2.4. SEM of floating-catalyst VGCF (ASI, 1999) ....................................... 21

2.5. SEM of a broken end of an as-grown VGCF (ASI, 1999) ........................... 22

3.1. Leistritz LSM 30.34 twin-screw laboratory extruder ................................ 28

3.2. Screw configuration used for the extrusion experiments ............................ 29

3.3. Extrusion process for uniform diameter composite strand ........................ 30

3.4. The dependence of flow rate on extrusion temperature at fixed screw rates



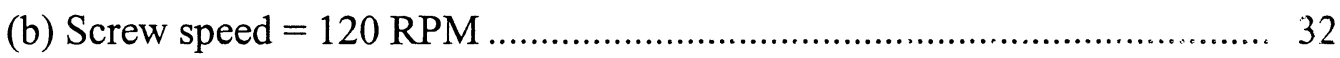

3.5. The dependence of pressure on extrusion temperature at fixed screw rates



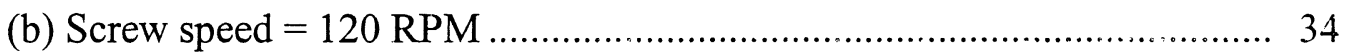

3.6. The dependence of flow rate and pressure on the screw speed at a constant extrusion temperature of $215^{\circ} \mathrm{C}$

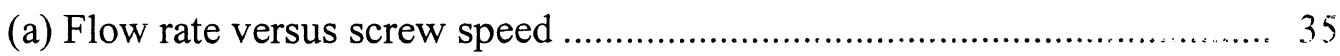

(b) Pressure versus screw speed .................................................. 36 
3.7. SEM of VGCF after ball-milling for 1 minute (Tibbetts and McHugh, 1999) ... 37

3.8. SEM of VGCF after the removal of the matrix from the extruded composite.... 38

3.9. Specifications of ASTM D638 Type II tensile specimen.............................. 40

3.10. Variation of ultimate tensile strength and modulus versus extrusion temperature at a constant screw speed of $40 \mathrm{RPM}$

(a) Ultimate tensile strength versus extrusion temperature...................... 42

(b) Tensile modulus versus extrusion temperature ............................... 42

3.11. Variation of ultimate tensile strength and modulus versus screw speed at a constant extrusion temperature of $215^{\circ} \mathrm{C}$

(a) Ultimate tensile strength versus extrusion temperature....................... 43

(b) Tensile modulus versus extrusion temperature ............................... 43

3.12. Experimental results for tensile strength of composite samples at various fiber

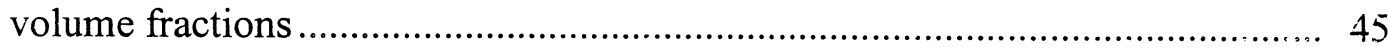

3.13. Experimental results for tensile modulus of composite samples at various fiber volume fractions

3.14. Variation of void content versus screw speed and temperature

(a) Void content versus screw speed with extrusion temperature at $215^{\circ} \mathrm{C} \ldots . .46$

(b) Void content versus extrusion temperature with screw speed at 40 RPM 47

3.15. SEM of clumps of VGCF (Tibbetts and McHugh, 1999) ............................ 48

3.16. Void content versus extrusion pressure of VGCF reinforced polypropylene .... 49

4.1. Transmission Laue method where the film is place behind the sample to record the beams which are transmitted through the sample.

4.2. A flat-film Laue pattern with the $(0,0,2)$ arcs of a carbon fiber 55 
4.3. An example of the diffraction planes of a composite sample with aligned fiber reinforcement

4.4. Side view of extruder die

4.5. Intensity versus azimuthal angle relative to the preferred axis of composite specimens

(a) $2.5 \% \mathrm{VGCF}$

(b) $7 \%$ VGCF

(c) $11 \% \mathrm{VGCF}$

4.6. Velocity profile for tube flow and the effect that shear flow has on fiber orientation.

4.7. Flow induced alignment of a fiber.

4.8. Side view of extruder die with an extended nozzle

4.9. Diffraction profile of a $2.5 \%$ volume VGCF reinforced specimen extruded through an extended nozzle.

5.1. Ultimate tensile strength of polypropylene composites reinforced with partially aligned and randomly oriented VGCF at various fiber volume fractions

5.2. Tensile modulus of polypropylene composites reinforced with partially aligned and randomly oriented at various fiber volume fractions.

5.3. Gaussian distribution function $F(\theta)$ of the fiber orientation with respect to axial direction. The axial direction is $\theta=0, Z$ is the full width of the diffraction intensity measured at one half the maximum intensity

5.4. The predicted ultimate tensile strengths of polypropylene reinforced with $15 \%$ volume $\mathrm{VGCF}$ as a function of fiber alignment and fiber length

5.5. The predicted UTS of a partially aligned VGCF reinforced polypropylene as a function of fiber volume fractions $(l=10 \mu \mathrm{m})$ and degree of alignment. 
5.6. Ultimate tensile strength of partially aligned VGCF reinforced polypropylene with various fiber volume fractions and various average fiber lengths. The experimental values are compared with the theoretical curve given by Eq.

6.1. Experimental density values of polypropylene reinforced with various amounts of Pyrograf III ${ }^{\mathrm{TM}}$ (PR-19-HT) VGCF. These values are compared with theoretical results calculated assuming the fiber density equal to that of Pyrograf I ${ }^{\mathrm{TM}}$ fiber

6.2. Experimental heat capacity values of polypropylene reinforced with various amounts of Pyrograf III ${ }^{\mathrm{TM}}$ (PR-19-HT) VGCF. These values are compared with theoretical results calculated assuming the specific heat of the fiber equal to that of Pyrograf I ${ }^{\mathrm{TM}}$

6.3. Experimental thermal diffusivity results in the longitudinal and transverse directions for the polypropylene composites at various fiber volume fractions.. 94

6.4. Experimental thermal conductivity in the longitudinal direction compared with the semi-theoretical Lewis and Nielsen model for uniaxially oriented fibers of different thermal conductivities $\left(k_{f}\right.$ in $\left.\mathrm{W} / \mathrm{m}-\mathrm{K}\right)$

6.5. Experimental thermal conductivity in the transverse direction compared with the semi-theoretical Lewis and Nielsen model for uniaxially oriented fibers of different thermal conductivities $\left(k_{f}=20 \mathrm{~W} / \mathrm{m}-\mathrm{K}\right)$.

6.6. Experimental thermal conductivity values of aligned, heat-treated fiber composites compared with results from other studies.

6.7. Electrical resistivity of the extruded composite strands and the molded cube samples with various fiber volume fractions 


\section{CHAPTER 1}

\section{INTRODUCTION}

\subsection{Background}

Composite materials have made significant advances in material applications for a variety of systems. They are comprised of two or more separate materials combined in a macroscopic structural unit that when combined, lead to improved properties over the individual components. Although many man-made materials have two or more constituents, they are not generally referred to as composites if the structural unit is formed at the microscopic level rather than the macroscopic level. Thus, metallic alloys and polymer blends are not usually classified as composites. The most common example is the fibrous composite consisting of reinforcing fibers embedded in a binder, or matrix material. Fibrous reinforcement is typically used because many materials are much stronger and stiffer in fiber form than they are in the bulk form. Therefore, the word "composite" usually refers to fiber-reinforced polymer, metal, and ceramic materials.

Fiber-reinforced composites were originally developed for the aerospace industry in the 1950's and can be broadly classified as continuous fiber and discontinuous fiber reinforced composites. Continuous fiber composites are manufactured using complex textile-like operations or fiber preform lay-up techniques where the fibers are arranged in the desired orientation and then impregnated with a resin to provide maximum strength along the fiber axis. These composites provide excellent strength properties in the aligned direction, but production is costly and labor-intensive, whereas discontinuous or 
short fiber composites can be fabricated into complex shapes using automated mass production methods, such as injection molding, compression molding, or extrusion. These materials consist of fibers that are generally straight and have uniform diameters in excess of 5 micrometers and lengths ranging from approximately $0.1 \mathrm{~cm}$ to $13 \mathrm{~cm}$ that are embedded in the polymeric matrix during processing. Since short fiber reinforced materials can be fabricated by automated equipment, they have become a commercially important class of engineering materials and exhibit the most potential for development and growth. Reinforcing plastics with fiber materials offers an increase in dimensional stability, strength, toughness, and resistance to harsh environmental conditions. Additional advantages include high strength-to-weight ratios, improved durability, and a unique flexibility in design capabilities.

A drawback of short fiber composites is that they are incapable of the strength properties possessed by continuous fiber materials. In composites, loads are not directly applied to the fibers but are applied to the matrix material and transferred to the fibers through the fiber ends and also through the cylindrical surface of the fiber near the ends. For continuous fiber composites the length of the fiber is much greater than the length over which the transfer of stress takes place, therefore the end effects can be neglected. Also, the fibers can be easily oriented along a particular direction in the matrix material. In the case of short fiber composites, mechanical and physical properties are functions of fiber length and orientation, while end effects significantly influence the behavior and reinforcing effects in the material. Thus, a critical fiber length must be achieved and 
alignment of the fibers is necessary to approach the strength capacity of continuous fiber reinforced composites.

There are currently many types of reinforcing fibers used in composite materials. Glass fibers are the most common reinforcing fibers for polymer matrix composites due to their low-cost and high strength, and are used in many high-volume applications, particularly the automotive industry. The disadvantages of glass fibers are that they have a relatively low modulus and poor abrasion resistance, which decreases its potential strength, and poor adhesion to polymer matrix resins, especially in the presence of moisture. Other composites made from carbon, aramid, boron, or other high modulus fibers are used primarily for more exotic aerospace and military applications where their higher costs can be justified by improved performance. Carbon fibers are currently the most widely used advanced fibers and are generally manufactured by the pyrolysis of a polyacrylonitrile (PAN) or a pitch precursor. They also have the highest specific strength and specific modulus of all reinforcing fiber materials. When compared to glass fibers, carbon fibers offer higher strength and modulus, lower density, outstanding thermal and electrical conductivity, but are much higher in cost. The high cost of producing carbon fibers is the principle barrier prohibiting carbon fiber reinforced composites from entering numerous commercial applications.

Recently, new manufacturing processes have been developed which produce carbon fibers at significantly lower costs. For example, a method has been introduced by Tibbetts et al. (1991) in which carbon fibers are catalytically grown by a vapor deposition process from hydrocarbons. The end product of this manufacturing process is a 
discontinuous mass of tangled microscopic carbon fibers. These nanometer-size fibers have a diameter of about 200 nanometers and a length ranging from 50 to 100 micrometers or longer. They are significantly smaller than conventionally available carbon fibers, which are generally on the order of 7 micrometers in diameter. These vapor grown carbon fibers (VGCF) are highly graphitic with superior mechanical properties and have high electrical and thermal conductivity. By being more economically feasible, the low-cost nanometer-size carbon fibers have the potential to override the cost barrier that has prevented conventional carbon fiber composites from entering into cost-sensitive commercial markets. These composites could find their way into numerous new markets, particularly the electronics, aerospace, and automotive industries.

Despite the potential benefits of nanometer-size VGCF, there are several significant disadvantages associated with using them for composite reinforcement. First, the microscopic fibers are entangled in a loosely packed fibrous matrix with an extremely low apparent bulk density of 0.001 to $0.02 \mathrm{~g} / \mathrm{cm}^{3}$. This makes them very difficult to handle and creates a potential health hazard from inhalation when they are dispersed into the air. Such a low density also makes it difficult to thoroughly blend the nanometer-size fibers with a polymeric matrix granules during preprocessing mixing. Also, because the basal graphite planes of the fibers are arranged in concentric circles to form a low energy surface, the VGCF is not readily wettable. Therefore, conventional matrix materials such as epoxies and polymers will not readily adhere to the fiber surface, such that mechanical properties of the composite are reduced because of voids and the lack of adhesion 
between the fiber and matrix. Finally, one of the greatest disadvantages of VGCF is that the fibers become randomly aligned and entangled during production.

Significant improvements have been, and are currently being made by the manufacturers of VGCF to improve the handling and processing issues of the fiber. For example, there are now post-processing pelletizing procedures, which increase the apparent bulk density of the fibers making them more manageable. Also, new surface treatment techniques have been developed to improve the wettability of the fibers. Sufficient adhesion between the polymeric matrix and the VGCF may now make it possible to indirectly measure the mechanical properties of the fibers. By better understanding the fiber properties, one can improve design and predict the behavior of the composite material. Indirect measurements are necessary because the VGCF is too small to allow the properties of the individual fibers to be measured directly, but prior efforts have been prevented because of the poor wettability of the fibers. Such advances in the processing of the nanometer-size VGCF have increased the potential of this product to new levels.

In spite of such improvements, the production of VGCF still yields a discontinuous random mass of entangled microscopic fibers. Therefore, the most promising applications for this material would be in composites where the reinforcement is randomly oriented throughout the composite, which produces isotropic strength properties. Random orientation is not always undesirable, but usually composites are designed to maximize performance along a given direction. To achieve this objective, alignment of the fibers is necessary. This is an important issue with regards to designing 
any short fiber reinforced polymer because numerous designs can utilize orientation to the advantage of performance.

Much work has been done to control orientation of short fibers in composite materials. Die design for an extrusion process can be altered to tailor the orientation of the fibers for particular applications. For instance, hollow extrudates such as tubing products whose structures involve stresses that require greater strength and stiffness in the circumferential direction can be produced to withstand such loading. Goettler et al. (1977) developed an extrusion process to obtain off axis orientation using a diverging die channel so that the outlet area is at least two or more times the inlet area of the die channel. This produced a substantial improvement in physical properties in the hoop direction. McPhee and Gray (1980) developed an extruder head that includes a doubled taper conical pin that orients fibers of an elastomer-fiber composite hose in the radial direction. Jarrin et al. (1994) also developed a die to improve burst strength by orienting the fibers in the circumferential direction. The method combines a proper formulation of material and a special geometry consisting of a diverging end zone on a conventional tube draw plate with a non-diverging straight section.

Numerous other fiber-aligning techniques have been developed for a broad range of products other than hollow extrudates. For example, Morgan (1989) reported an extrusion apparatus having parallel top and bottom surfaces that converges and has a diamond-shaped flow bar for aligning fibers during extrusion. This method provided substantial fiber alignment across a wide extrudate. Miani (1995) used a two-component extrusion head having a spinneret with high perforation density and a distribution unit for 
introducing two reinforcement components. An extrusion die for the extrusion of continuous fiber reinforced plastics was developed by El-Sobky (1999). A twin screw extruder was used by Michel (1991) to process a continuous carbon fiber reinforced fluoropolymer composite mixture, and by Sargent (1995) to compound thermoplastic resin materials with randomly oriented fibers. A method to control the orientation of chopped fibers by subjecting the composite mixture to a drawing treatment was introduced by Daimaru (1990). Finally, Tepic (1992) utilized sound waves and Knoblachs (1991) applied electric fields to orient fibers suspended in a matrix of a composite material.

Since VGCF is generated in a different form than conventional carbon fibers, standard composite processing methods may need to be modified to accommodate VGCF composites. Dasch et al. (1993) introduced a method to manufacture a VGCF composite by combining the carbon fibers with both polycarbonate and nylon resins. The mixtures were first vacuum dried at an elevated temperature and then mixed in a Brabender mixing bowl at temperatures $10^{\circ}$ to $20^{\circ}$ above the melting point of the resins. The material was then compression molded into test specimens. A similar procedure was used by Dasch and Alig (1995). The results showed an increase in tensile and flexural strength from the matrix strength for volume fractions below $30 \%$. The tensile modulus and flexural modulus also increased with fiber content. Caldeira et al. (1998) and Cameiro et al. (1998) also studied polycarbonate composites reinforced with VGCF. Both of their findings showed that the tensile properties of the polycarbonate were marginally 
improved by the addition of VGCF, and that the impact resistance decreased by the addition of the fibers.

VGCF composites with epoxy and poly(phenylene sulfide) matrices was studied by Patton et al. (1999). This work demonstrates that mixing is a very important variable in determining the quality of VGCF composites. Increases in stiffness and flexural strength demonstrated that the discontinuous randomly oriented VGCF has great potential for composite reinforcement. Yamanashi et al. (1993) also analyzed the properties of epoxy composites embedded with VGCF. Their tests showed that the tensile shear strength of VGCF reinforced epoxy composites are far superior to conventional shortfiber carbon reinforcement.

Tibbetts and McHugh (1999) showed that the mechanical properties of VGCF reinforced thermoplastics could be improved by ball-milling the fibers before blending them with the polymer matrix. This breaks down the fiber clumps and increases the infiltration of the matrix material during processing (injection molding). It was also shown that infiltration of the fibers could be improved by processing under higher pressures. The addition of $11.5 \%$ by volume VGCF approximately doubled the tensile strength and modulus of pure polypropylene. Van Hattum et al. (1999) also used a polypropylene matrix to investigate the properties the composites reinforced with PAN fibers and VGCF. The strength of the VGCF reinforced composites was similar to those reinforced with the PAN fibers, however the stiffness was marginally lower. Zhang et al. (1998) have reported on the morphology and electrical properties of VGCF/HDPE composites. 
It can be concluded that it is possible to produce a viable randomly oriented VGCF composite. However, the issue of anisotropy due to fiber orientation was not addressed until Dutta et al. (1995) attempted to extrude a polycarbonate sheet with aligned VGCF. The composite blends were extruded through a rectangular shear die by a single screw plasticating extruder. Uniaxial alignment of the fibers along the extrusion direction was produced by melt-drawing the film as it exited the die. The anisotropic films could then be used as prepegs to fabricate laminated composites. Their results showed that it was difficult to disperse the VGCF because the fibers tend to agglomerate, especially as fiber content increases; thus inhibiting fiber alignment. Ii was suggested by Dutta et al. (1995) that fiber dispersion could be improved by starting with a more homogeneous dry composite mixture and melt blending the components in a rnixer before extrusion.

\subsection{Scope of the Dissertation}

The processing of VGCF composites is relatively new and is still in the research and development stage. As mentioned earlier, there are still many unresolved problems involving VGCF, namely the fibers are microscopic in size and become randomly aligned and entangled during production. These characteristics help restrict the commercial utilization of the fiber; however, if the fiber could be dispersed and aligned in a matrix suspension, they could find their way into a multitude of new commercial markets and applications. Therefore, the primary focus of this work is to introduce an extrusion method to produce an aligned VGCF composite. 
A twin-screw extruder will be used to process the composite material and a specialized die designed to generate a high degree of flow-induced alignment of the nanometer-size VGCF will be utilized. The VGCF will be processed in a polypropylene matrix. Polypropylene was chosen as the matrix material used throughout this study because earlier work has shown that polypropylene produces viable composites when reinforced with VGCF. Studies carried out by Tibbetts (2000) have shown that polypropylene readily wets and provides a strong interfacial bond with VGCF. The work done by Carneiro et al. (1998) also stated that using a semi-crystalline polymer such as polypropylene might improve composite properties, as compared with other matrix materials such as polycarbonate or nylon. Since polypropylene has a surface energy closer to that of VGCF the composite product is also less prone to chemical stress cracking.

In order to develop VGCF composites, it would be helpful to understand the processing and characteristics of VGCF, and how they differ from conventional carbon fibers. This is reviewed in Chapter 2, where the textile-like production methods of PAN and pitch-based carbon fiber are introduced. The production of VGCF by the pyrolysis of a hydrocarbon gas in the presence of a catalyst is then discussed.

To better understand the extrusion process of the nanometer-size VGCF reinforced polypropylene, the influence of extrusion conditions such as temperature and screw speed, and strength properties of the composite products are examined in Chapter 3. The fiber length degradation caused by the extrusion process and the void content of the specimens are also examined. 
In Chapter 4, the method for fabricating an aligned VGCF reinforced polypropylene composite is introduced. A specially designed die configuration is used to generate fiber alignment along the extrusion direction. It will be shown that fiber alignment can be improved by increasing the residence time in the die channel. The degree of fiber alignment is quantified using x-ray diffraction. The mechanical properties of the aligned composites are compared with experimental results of randomly oriented VGCF composites.

The work presented in Chapter 5 introduces an experimental and theoretical approach for determining the strength of partially aligned discontinuous fiber reinforced composites. The fiber alignment information is obtained as a Gaussian or normal distribution function by using $\mathrm{x}$-ray diffraction. The distribution function is then used in the composite strength equation to calculate the theoretical strength. This approach is then applied to the VGCF reinforced polypropylene composites, and the experimental and theoretical results are then compared.

The transport properties of the aligned fiber reinforced polypropylene composites are investigated in Chapter 6. Both the thermal diffusivity and electrical resistivity of specimens reinforced with heat-treated VGCF are measured in both the longitudinal and transverse directions. The density and specific heat are also determined and used to calculate the thermal conductivity. The thermal conductivity results are compared with theoretical predictions and other composites containing similar fiber.

The conclusions and suggestions for further study of this work are summarized in Chapter 7. 


\section{CHAPTER 2}

\section{CARBON FIBERS}

\subsection{Introduction}

Carbon fibers and filaments are not new; Edison first produced them in 1879 when he took out a patent for the manufacture of carbon filaments suitable for use in electric lamps. However, it wasn't until the 1970's when successful commercial production was initiated to meet the demands of more exotic applications such as aerospace structures, where higher cost can be justified based on improved performance.

The major advantages of carbon fibers over glass fibers and conventional bulk metallic materials are higher modulus and lower density. In many applications such as aerospace and automotive structures, structural weight is very important. Depending on whether the structural design is strength-critical or stiffness-critical, the material used should have a high strength-to-weight ratio or a high stiffness-to-weight ratio. Carbon fibers and their composites have a tremendous advantage over conventional materials in this area. This is the principle reason that carbon fiber reinforced composites are being used with increasing frequency for aerospace and automotive structures.

Carbon can occur in three forms: diamond, graphite, and amorpholis. Only the crystalline forms (diamond and graphite) have high modulus. Diamond has a threedimensional covalent bonded structure and graphite has a two-dimensional covalent bonded structure. In the graphite structure, the carbon is arranged in the form of hexagonal layers. Unfortunately, the name "carbon" and "graphite" are often used 
interchangeably to describe fibers based on the element carbon. These fibers are usually produced by subjecting organic precursor fibers to a sequence of heat treatments so that the precursor is converted to carbon by pyrolysis. The major difference is that graphite fibers are subjected to higher temperature pyrolysis than carbon fibers. The result is that carbon fibers are typically less than $95 \%$ carbon, whereas graphite fibers are at least $99 \%$ carbon (Gibson, 1994).

Although carbon fibers were once prohibitively expensive, the cost has dropped significantly as the production capacity and demand has increased over the past 20 years. The development of new carbon and graphite fibers continues at a rapid pace. The current technology for producing carbon fibers generally centers on the thermal decomposition of various organic precursors. It is basically the heat treatment of the precursor that removes the oxygen, nitrogen, and hydrogen to form carbon fibers. It is well established in carbon fiber literature that the mechanical properties of the carbon fibers are improved by increasing the crystallinity and orientation, and by reducing the defects in the fiber.

Candidate organic materials for pyrolysis into carbon fibers having good properties should possess the appropriate strength and handling characteristics needed to hold the fibers together during all stages of the conversion process to carbon Also, the precursor should not melt during any stages of the conversion process. This is accomplished buy stabilizing the thermoplastic precursor prior to the process. The precursor material must not completely volatilize during the pyrolysis process; that is, the carbon yield of the precursor fiber after pyrolysis should be appreciable enough to justify 
its use on an economic basis. Furthermore, in order to obtain optimal properties, carbon atoms should tend to array themselves in an aligned graphite structure during pyrolysis. Finally, the precursor material should be as inexpensive as possible (Agarwal and Broutman, 1990). A large variety of precursor materials are used to produce carbon fibers of different morphologies and specific characteristics. Currently, the two primary precursors used for carbon fiber production are Polyacryonitrile (PAN) and pitch. Pitch is made by coal tar distillation or from high molecular residues in crude-oil distillation.

\subsection{PAN and Pitch Derived Carbon Fibers}

PAN and pitch derived carbon fibers are the most commonly produced carbon fibers and are used in a wide variety of composite materials and applications. Both fibers are manufactured by similar processes and are available in various forms: continuous, chopped, woven fabric, or mat. The production methods for graphite fibers derived from PAN and pitch are described below in the following subsections.

\subsubsection{Graphite Fibers from PAN}

The process by which PAN is converted to carbon fibers involves several steps:

1. Spinning the PAN into a precursor fiber.

2. Stretching the precursor.

3. Stabilization by holding the prestretched polymer under tension at a temperature of $205-240^{\circ} \mathrm{C}$ for up to 24 hours in an oxidizing atmosphere such as air. 
4. Carbonization at approximately $1500^{\circ} \mathrm{C}$ in an inert atmosphere. Carbonization is the process of pyrolizing stabilized PAN fibers to drive out most, if not all, noncarbon elements from the precursor fibers until they are essentially transformed into carbon fibers. It is during this stage that the high mechanical properties found in most commercially available fibers are developed.

5. Graphitization at approximately $3000^{\circ} \mathrm{C}$ in an inert atmosphere. Graphitization heat treatments are carried out at temperatures in excess of $1800^{\circ} \mathrm{C}$ in order to improve the tensile modulus of the fiber by improving crystallite structure and preferred orientation of the graphite crystallite within each individual fiber.

6. Surface treatment by wet oxidation, dry oxidation, or anodic oxidation. The function of reinforcing fiber is fulfilled only when enough stress transfer from matrix to fiber and vice versa is ensured by the adhesion and physical compatibility between fibers and matrix. Surface treatment influences the degree of adhesion by forming oxides on the surface of the carbon fibers.

\subsubsection{Graphite Fibers from Pitch}

The method by which pitch is converted to carbon fibers is very similar to the PAN process, and is as follows:

1. The pitch is melt spun at high strain rates to align the molecules parallel to the fiber axis and extruded through spinnerettes then rapidly cooled. 
2. Stabilization by holding the prestretched polymer under tension at a low temperature $\left(<100^{\circ} \mathrm{C}\right)$ for up to 24 hours in an oxidizing atmosphere such as air to ensure stabilization of the fiber by cross-linking and rendering it infusible.

3. Carbonization at approximately $1000^{\circ} \mathrm{C}$ in an inert atmosphere. However upon carbonization of pitch fibers, relaxation of the molecules takes place, which produces fibers with no significant preferred orientation and is the cause for poor mechanical properties of the carbon fiber product.

5. Graphitization at approximately $2500^{\circ} \mathrm{C}$ in an inert atmosphere. Graphitization to improve the crystallite structure and preferred orientation of the graphite crystallite to increase the tensile modulus and strength properties of the fibers.

6. Surface treatment by oxidation methods that form oxides on the surface of the carbon fibers. This assures adhesion and physical compatibility between fibers and matrix.

Both fibers require textile-like operations for production of the continuous fibers. The processes are very similar except for the initial stages of the production operation. For the PAN fiber, the coiled macromolecule precursor is stretched in the direction of the polymer chain parallel with the fiber axis, thus transforming the linear molecule into a ladder polymer. And the preferred orientation of the pitch fiber is accomplished by spinning the liquid crystals consisting of polyaromatic molecules into the direction parallel with the fiber flow. The process flow diagram shown in Fig. 2.1 compares the two fiber production methods. 


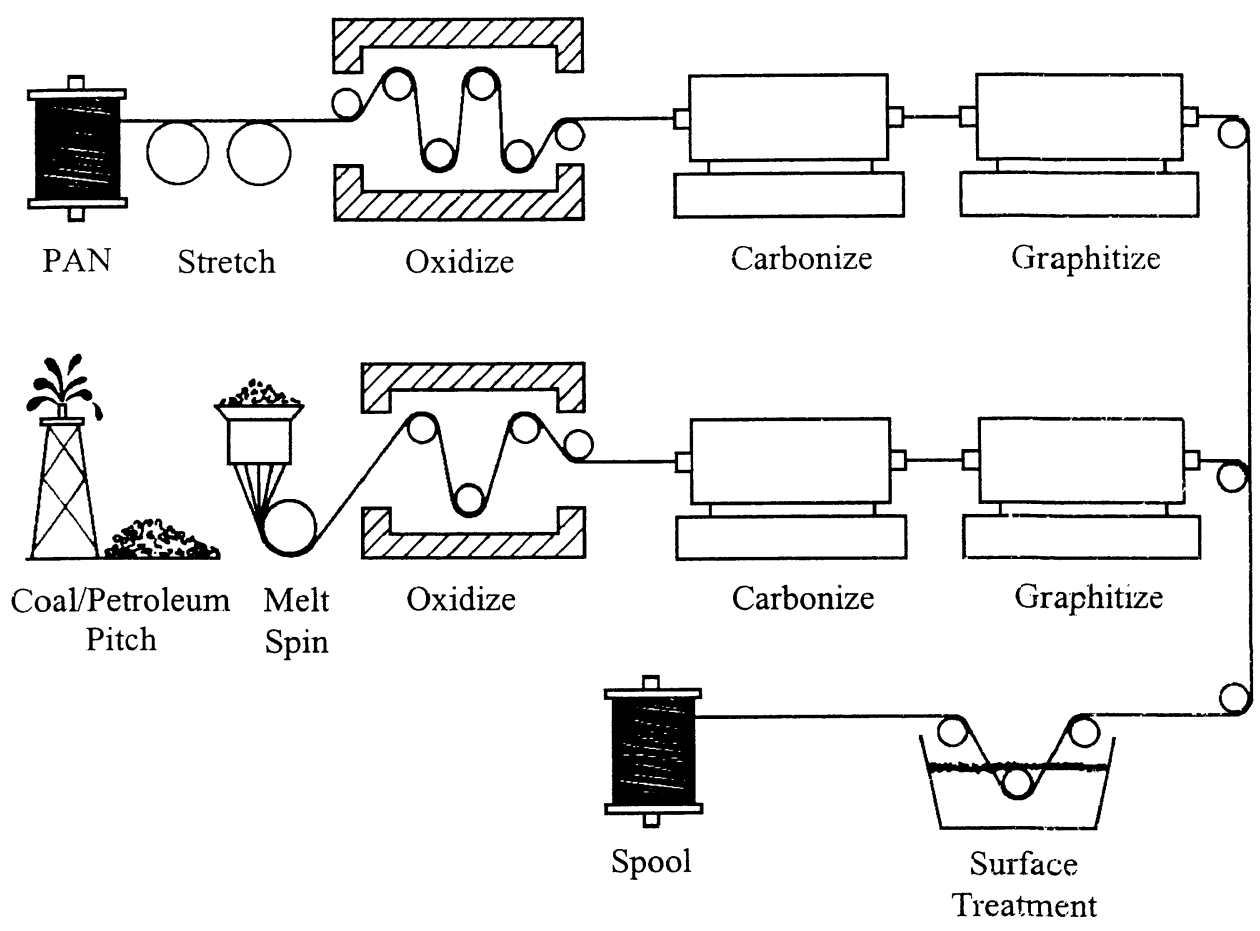

Fig. 2.1. Comparison of PAN and pitch processes for carbon fiber production (Bunsell, 1988).

Each process utilized to produce these carbon fibers offers distinct advantages and drawbacks in terms of both cost and properties. The PAN process results in carbon and graphite fibers with excellent strength properties. Where as pitch-based carbon fibers are of lower quality and inferior mechanical properties, but are currently the lowest-cost fiber on the market. Even though the cost of carbon fiber has significantly decreased over the past decade, they are still relatively expensive compared with industrial standards. These high fiber costs and the complexity of existing fabrication technologies prevent widespread commercial use of high-performance advanced composites composed of a polymer matrix and continuous carbon fiber reinforcements. Recently, a new grade of 
low cost carbon fiber has been developed in hopes of alleviating this problem. This advanced technology of an old concept is known as vapor grown carbon fiber.

\subsection{Vapor Grown Carbon Fiber}

Vapor grown carbon fiber (VGCF) is a new class of carbon fiber that is distinctively different from other types of carbon fiber in its method of production, its unique physical characteristics, and the prospect of low-cost fabrication. Due to their low cost and superior graphitic structure, VGCF offers a unique opportunity for carbon fiber composites to expand into a multitude of new markets (Fig. 2.2).

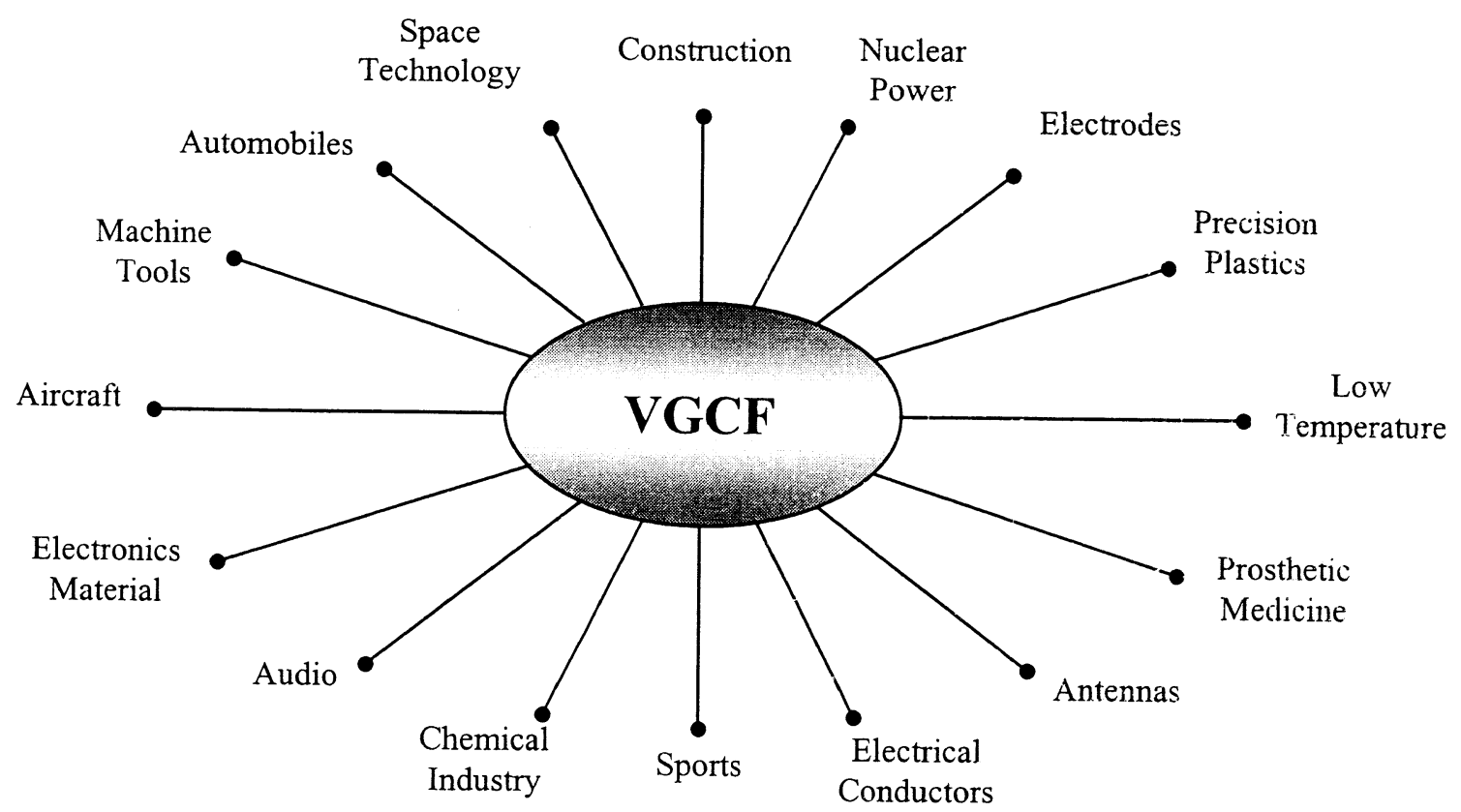

Fig. 2.2. Schematic illustration of expected applications of VGCF. 
The history of growing carbon fibers from hydrocarbon gases is more than 100 years old (Hughes and Chambers, 1889), but the detailed mechanisms of fiber formation and growth are still not completely understood. Since then, there have been many attempts to explore the underlying principles behind VGCF production. Koyama (1972), and later Koyama and Endo (1973) made significant developments in this area by studying the decomposition of benzene at $1200^{\circ} \mathrm{C}$. Katsuki et al. (1981) grew fibers on many types of catalyst particles by thermally decomposing naphthalene-hydrogen mixture. Endo et al. (1983) investigated the relationship between the fiber structure and the catalytic particles that initiate and enhance the growth of fibers. Masuda et al. (1993) studied the liquid pulse injection technique for producing VGCF.

Vapor grown carbon fibers may be produced in a two-stage batch process where the length of the fiber can vary from about 100 micrometers to severai centimeters or longer, and a diameter up to 100 micrometers (Tibbetts and Devour, 1986). In the batch process the fibers are prepared by decomposition of hydrocarbon on a catalyst seeded substrate. This version of VGCF is called Pyrograf I ${ }^{T M}$ by Applied Sciences, Inc. (ASI Cedarville, $\mathrm{OH}$ ). It should be noted that the primary production of VGCF in the United States is carried out by ASI. Patent rights have been issued to ASI and 'Pyrograf' is the registered trademark name given to their VGCF. Another variety of VGCF from ASI, Pyrograf III ${ }^{T M}$, is much smaller than the Pyrograf $I^{T M}$ fiber and is produced from a floating-catalyst in a continuous-flow reactor (Tibbetts et al., 1991 and 1993). This onestage technique produces fibers at a much higher rate and at lower costs than the twostage process used for the Pyrograf $I^{\mathrm{TM}}$ fiber. It has been projected that the Pyrograf III ${ }^{\mathrm{m}}$ 
fiber can be produced for as little as $\$ 3$ per pound, opposed to $\$ 40$ per pound for low modulus PAN derived fibers. The low production costs of the Pyrograf IIITM VGCF offers a unique opportunity for carbon fiber composites to expand into numerous new markets, and exploiting such composites is the driving force behind this study.

The floating catalyst technique for producing the Pyrograf III ${ }^{T M}$ VGCF was developed by Tibbets et al. (1991), and employs a catalyst in the gas phase transported into a heated reactor with a hydrocarbon gas (Fig. 2.3).



Fig. 2.3. Process flow diagram for VGCF.

The pyrolysis of hydrocarbon in the presence of the catalyst results in the formation of a graphitic fibril. The filaments are thus generated in an airborne 
configuration, and drift towards the gas exit driven by the reactant gas flow. As the fibers intersect in the gas stream they may become tangled, thus resulting in an entangled mass of discontinuous randomly oriented fibers (Fig. 2.4). Also, since the residence times are short in the continuous flow reactor, the fibers are usually less than 100 micrometers in length and 200 nanometers in diameter.

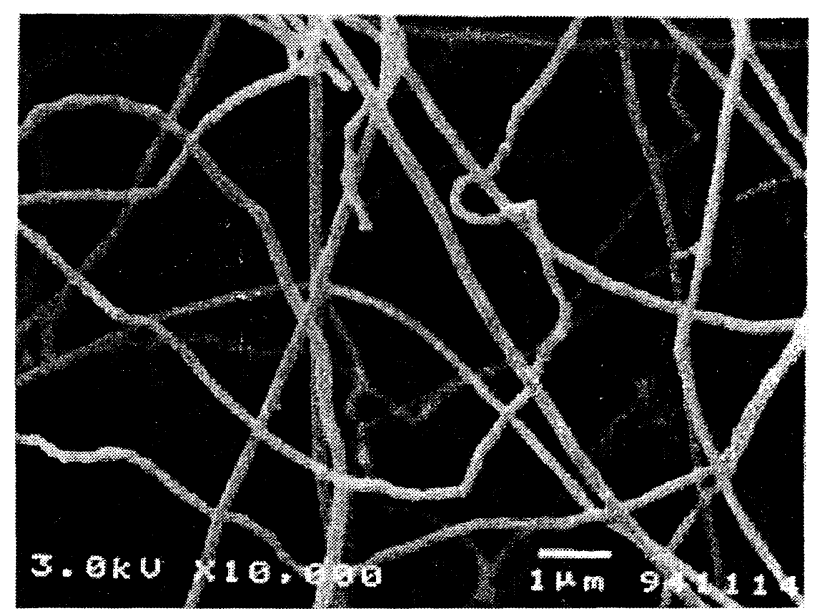

Fig. 2.4. SEM of floating-catalyst VGCF (ASI, 1999).

This randomly oriented and entangled mass of fibers is a characteristic that makes this fiber difficult for many composite fabrication processes. Therefore, the most promising applications for this material would be randomly oriented reinforced composites. But if these fibers could be untangled and aligned in a polymer flow, numerous new low-cost discontinuous fiber reinforced composites with specific strength properties could be produced. 
With addition to low production costs, VGCF has many preferential qualities when compared with PAN and pitch based carbon fibers. The morphology of VGCF is unique in that the graphene planes are more preferentially oriented around the axis of the fiber. Figure 2.5 is a scanning electron micrograph (SEM) of the broken end of a thick VGCF which shows the successive layers of chemical vapor deposited carbon, resulting in nested graphene planes.

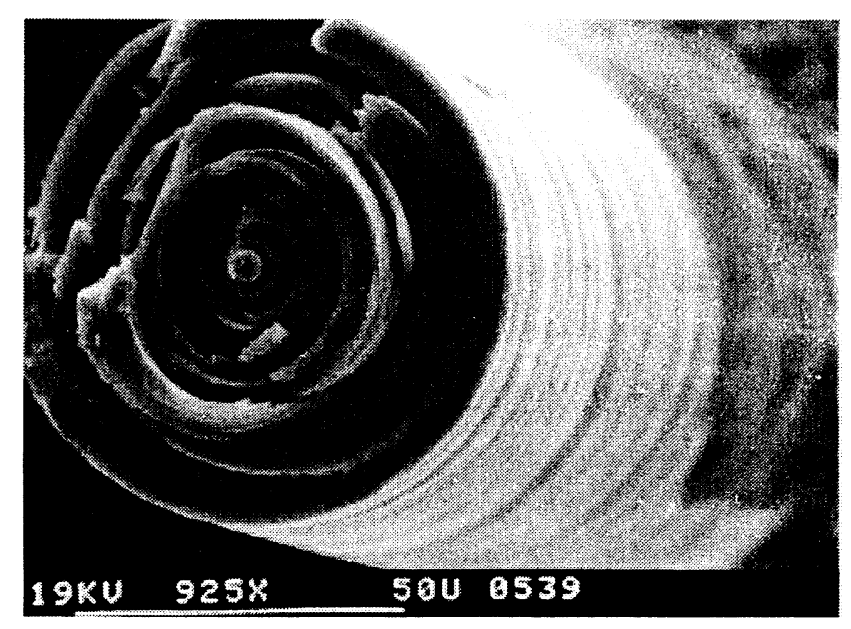

Fig. 2.5. SEM of a broken end of an as-grown VGCF (ASI, 1999).

As would be expected, the properties of VGCF are strongly influenced by this morphology. Also, because the formation of the core fibril by diffusion through a catalyst particle and subsequent chemical vapor deposition of carbon on the surface of the fibril favors carbon deposition of relatively high purity, VGCF may be highly graphitized with a heat-treatment of about $2800^{\circ} \mathrm{C}$. Consequences of the circumferential orientation 
of high purity graphene planes is a lack of cross-linking between the graphene layers, and a relative lack of active sites on the fiber surface, making it more resistant to oxidation, and relatively unreactive to matrix materials. Also in contrast to carbon fiber derived from PAN or pitch precursors, VGCF is produced only in a discontinuous form, where the length of the fiber can be varied from about 20 microns to several centimeters. This fact has significant implications with respect to composite fabrication, since the textile handling methods used for continuous carbon fibers derived from PAN and pitch are not immediately applicable to VGCF.

The small size of VGCF makes it difficult to perform direct measurements for determining the bulk properties of the fibers. One approach for estimating these properties is by growing the VGCF (using the batch process) in the laboratory to a size large enough to test with standard equipment. This approach was taken by Tibbetts and Beetz (1997) to estimate the properties of the VGCF with diameters in excess of 5 micrometers. This technique was used to determine the properties of the larger Pyrograf $I^{\mathrm{TM}}$ fiber (Table 2.1).

The work of Tibbetts and Beetz (1997) also found that strength properties increased with decreasing diameter. Since the diameters of the fibers used in the tests were far greater than the average diameter of 0.2 micrometers for the Pyrograf III'M fiber, the strength and stiffness of the Pyrograf III ${ }^{T M}$ fiber could not be estimated from these results. This also holds true for estimating other properties of the fibers. 'Therefore, the actual properties of the Pyrograf III ${ }^{\mathrm{TM}}$ fiber remains uncertain; however, up to this point, most 
researchers have been using the values found for the Pyrograf $I^{\mathrm{TM}}$ fiber (Table 2.1) to approximate that of the Pyrograf III ${ }^{T M}$ fiber.

Table 2.1. Properties of the Pyrograf $I^{T M}$ fiber (ASI, 1999).

\begin{tabular}{ccc}
\hline Property & As-Grown & Heat-Treated \\
\hline Filament Diameter, $\mu \mathrm{m}$ & 0.1 to 100 & 0.1 to 100 \\
Tensile Strength, GPa & 2.7 & 7.0 \\
Tensile Modulus, GPa & 400 & 600 \\
Break Elongation, $\%$ & 1.5 & 0.5 \\
Density, g/cm ${ }^{3}$ & 1.8 & 2.1 \\
Electrical Resistivity, $\mu \Omega-\mathrm{cm}$ & 1000 & 55 \\
Thermal Conductivity, $\mathrm{W} / \mathrm{m}-\mathrm{K}$ & 20 & 1950 \\
\hline
\end{tabular}

\subsection{Types of Pyrograf III ${ }^{\mathrm{TM}}$ VGCF}

Three kinds of the nanometer-size Pyrograf III ${ }^{T M}$ fiber are used throughout this study and supplied by Applied Sciences, Inc. The first type is labeled PR-21-AG, and is used for the work performed in Chapter 3. This as-grown fiber was pelletized for handling purposes, but was not post-processed for surface treatment. The second variety labeled PR-21-PS was post-processed to enhance interfacial bonding and physical compatibility between fibers and matrix, thus providing high mechanical strength in composites. This was done by pyrolytically stripping the surface of the fiber in carbon dioxide. The PR-21-PS fiber is used for the experiments performed in Chapters 4 and 5. 
It should be noted that the PR-21-AG and the PR-21-PS fibers are very similar in appearance, and both have a bulk density of approximately $56 \mathrm{~kg} / \mathrm{m}^{3}$. The final type of Pyrograf $\mathrm{IIIM}^{\mathrm{TM}}$ used is the PR-19-HT fiber. This fiber was heat-treated at $3000^{\circ} \mathrm{C}$ to improve the graphitic crystallite structure of the fiber, thus increasing the conductive properties of the fiber and it's composites. Therefore, the PR-19-HT fiber is used in Chapter 6 to study the transport properties of their composites. This fiber was debulked using the Littleford Day process, thus increasing the bulk density to approximately 200 $\mathrm{kg} / \mathrm{m}^{3}$. The three varieties of Pyrograf IIITM described here are similar in size; with the diameter of the fibers approximately 200 nanometers and lengths varying from 20 to 80 micrometers. 


\section{CHAPTER 3}

\section{EXTRUSION CONDITIONS AND PROPERTIES OF VGCF REINFORCED POLYPROPYLENE}

\subsection{Introduction}

Understanding the extrusion process of VGCF reinforced composites is critical in order to process the composite into new products. In the extrusion process, conditions such as temperature, screw speed, and fiber volume have an affect on the bulk properties of the extrudate. Therefore, the purpose of this work presented in this chapter is to evaluate the various extrusion conditions and the resultant properties of $\mathrm{VGCF}$ reinforced polypropylene to determine the optimal processing parameters for the iwin-screw extrusion process.

Temperature, screw speed, feed rate, and fiber volume fractions are the controllable variables in the extrusion process. Therefore, the effects that these parameters have on the extrusion process such as flow rate, extrusion pressure, strength and stiffness, and void content will be found by performing several experimental runs. To do this, several composite mixtures containing various amounts of the PR-21-AG fiber will be fed into the extruder at a constant rate, which is kept proportional to the screw speed, thus eliminating one variable (feed rate). It should be noted that the feed rate needs to be approximately proportional to screw speed for the process to be stable This leaves temperature, screw speed, and fiber volume fraction as independent variables; which will be varied to understand how they affect flow rate, die pressure, strengthı and stiffness, and void content (dependent variables) at various fiber volume fractions. 
Preliminary runs have shown that the feasible processing temperatures for the fiber/polymer mixtures range from $215^{\circ} \mathrm{C}$ to $250^{\circ} \mathrm{C}$, whereas, screw speeds range from 40 to 120 RPM. Based on these preliminary results the input parameters selected for the experiments are shown below in Table 3.1.

Table 3.1. Input parameters for experiments.

\begin{tabular}{cccc}
\hline Input Parameters & \multicolumn{3}{c}{ Process Parameter Settings } \\
\hline Temperature & $215^{\circ} \mathrm{C}$ & $235^{\circ} \mathrm{C}$ & $250^{\circ} \mathrm{C}$ \\
Screw Speed & $40 \mathrm{RPM}$ & $80 \mathrm{RPM}$ & $120 \mathrm{RPM}$ \\
* Fiber Volume $\%$ & $2.5 \%$ & $7.5 \%$ & $12.5 \%$ \\
\hline
\end{tabular}

* Base-line runs with fiber volume fraction $=0 \%$ are also made

Two types of output parameters will be measured as shown in Table 3.2. Flow rate and pressure are process parameters that depend on the input parameters. The dependence of these process parameters on the input parameters will be determined.

Table 3.2. Output parameters for experiments.

\begin{tabular}{cccc} 
& \multicolumn{3}{c}{ Output Parameters } \\
\cline { 2 - 4 } Process Outputs & Flow Rate & Pressure & - \\
Products & UTS & Modulus & Void \% \\
\hline
\end{tabular}




\subsection{Composite Processing}

The procedure to produce a VGCF reinforced polypropylene composite begins by drying the fibers in a vacuum oven at approximately $300^{\circ} \mathrm{C}$ for 3 hours to assure the removal of moisture. The fibers are then dry-mixed at room temperature in a dual-shell dry blender with Pro-Fax 6301, a polypropylene homopolymer in powdered form manufactured by Montell U.S.A., Inc., until a homogeneous mixture is formed. The composite processing is initiated by feeding the granulated mixtures into an intermeshing, counter-rotating, modular Leistritz LSM 30.34 twin-screw laboratory extruder shown below in Fig. 3.1.

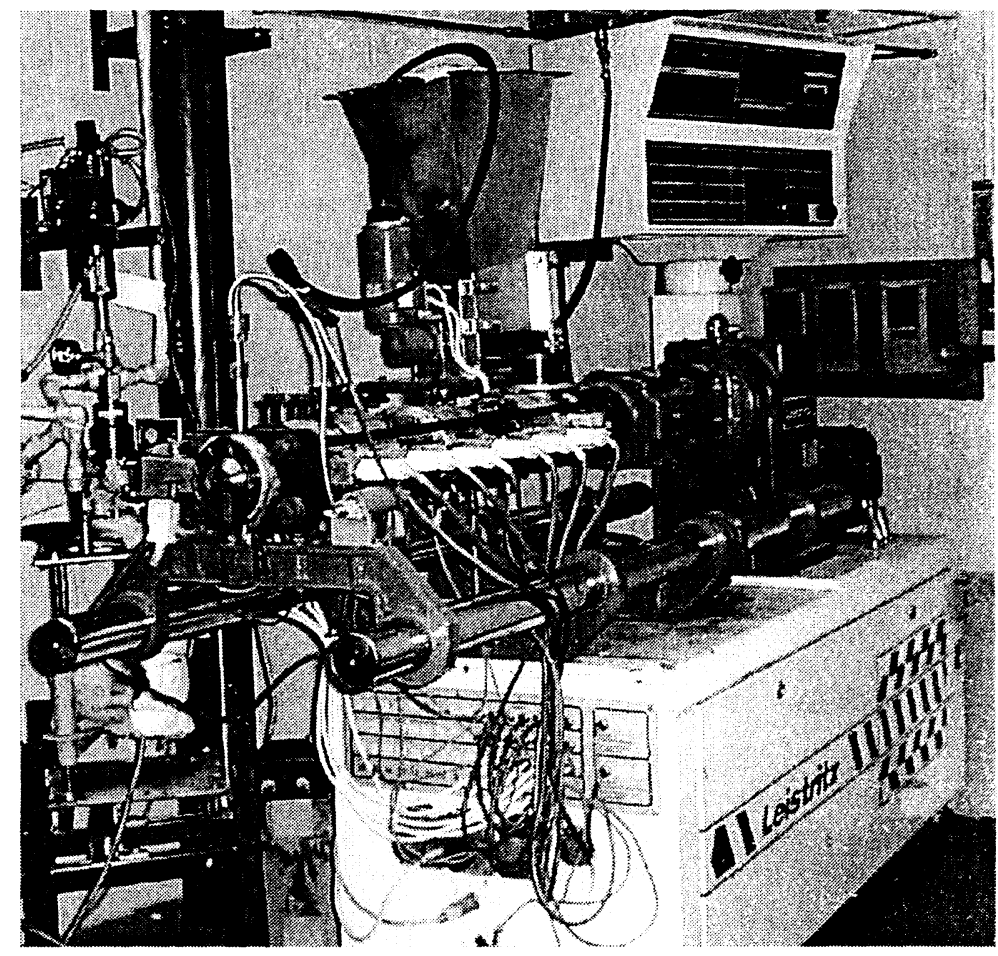

Fig. 3.1. Leistritz LSM 30.34 twin-screw laboratory extruder. 
The extruder has six-barrel elements with a heating and air cooling system. A venting type screw configuration consisting of A) a feeding zone, B) a compression zone, C) a conveying zone, D) a shearing zone, E) a venting zone, and D) a metering zone was adopted (Fig. 3.2). A vacuum pump was attached to the venting block to remove volatiles. Earlier experiments at Ohio University have shown that this screw configuration works successfully for VGCF composites.

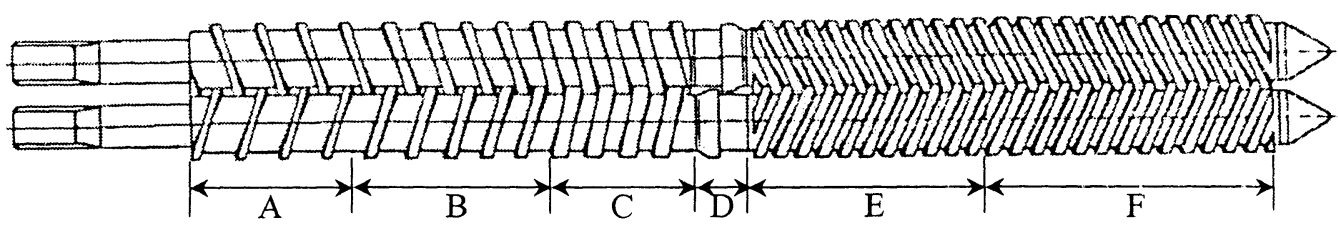

Fig. 3.2. Screw configuration used for the extrusion experiments.

A rotary-screw-metering feeder was used to control the feed rate, and a round die with a $2 \mathrm{~mm}$ opening was used to produce a continuous composite strand. 'The composite strand was kept in constant tension (with minimal reduction in the $2 \mathrm{~mm}$ strand diameter) while solidifying in air by natural convection until it was pulled into the fiber winder. In order to extrude a uniform diameter composite strand with a good surface finish, it was necessary to increase the die temperature as the fiber content increased. For instance, the die temperature was held at $180^{\circ} \mathrm{C}$ for the composite mixtures containing $2.5 \%$ fiber volume, and was increased to $200^{\circ} \mathrm{C}$ for the $12.5 \%$ vol. composites. The barrel temperature was one of the variables in the experiments. Therefore, all the different fiber 
content composites were extruded at three different barrel temperatures of $215^{\circ} \mathrm{C}, 232^{\circ} \mathrm{C}$, and $250^{\circ} \mathrm{C}$.

The process is continuous and is illustrated in Fig. 3.3. The continuous composite strands embedded with VGCF can now be reprocessed in such a way to form a functional composite component with directional reinforcement. This can be done by any suitable means, including hot-pressing and compression molding techniques.

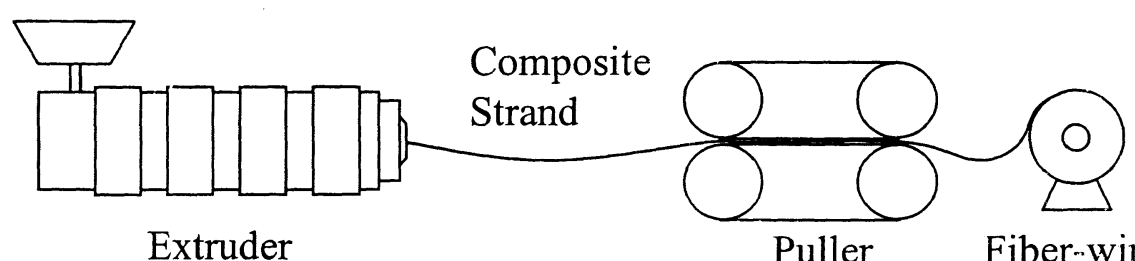

Extruder

Puller

Fiber-winder

Fig. 3.3. Extrusion process for uniform diameter composite strand.

\subsection{Variation of Extrusion Conditions on Flow Rate and Pressure}

To accurately compare how the extrusion flow rate and pressure are affected by the variation of the screw speed, temperature and fiber content, a controlled feed rate of the composite mixtures in different ratios must be achieved. Since the VGCF has such a low bulk density, it is extremely difficult to obtain consistent feed rates with a mixture of the raw fiber and polymer powder. Therefore, the fiber/polymer mixtures were compounded into solid pellets. The compounding was performed using the same screw configuration as previously described with a melt-flow temperature of approximately $290^{\circ} \mathrm{C}$ and a screw speed of $25 \mathrm{RPM}$. The temperature of the extruder die was set at 
$190^{\circ} \mathrm{C}$. Since the pellets were in a consolidated form, it was now possible to feed the extruder at a controlled rate for each mixture as the extruder screw speed is increased from 40 to 120 RPM. A rotary-screw-metering feeder was used to control the delivery of the pelletized feedstock. A stable feed rate was first achieved at the base screw speed of 40 RPM for each of the composite mixtures containing $0 \%$ to $12.5 \%$ volume fiber. The feed rate was then kept proportional to the screw speed. Therefore, the screw speed is an indication of the feed rate for the experiments. Since the feed rate and extrusion flow rate must be the same during the extrusion process, the flow rate must be linearly dependent on the screw speed; this was checked during analysis of the results.

The variation of fiber content, extrusion temperature, and screw speed has a direct affect on the flow rate and pressure during the extrusion of VGCF reinforced polypropylene. The effects of these parameters on the extrusion process are shown in the following figures. As the amount of fiber is increased in the polymer melt, the flow rate tends to go down. This is shown in Fig. 3.4(a), where it is observed that the flow rate at $40 \mathrm{RPM}$ screw speed and $232^{\circ} \mathrm{C}$ decreases from $1.7 \mathrm{~cm}^{3} / \mathrm{sec}$ to $0.97 \mathrm{~cm}^{3} / \mathrm{sec}$ as fiber content increases from pure polypropylene (PP) to $12.5 \%$ by volume VGCF. A similar trend is observed in Fig. 3.4(b) at a higher screw speed of 120 RPM. This result will be discussed later along with the effect of VGCF content on the extrusion pressure.

In Figs. 3.4(a) and 3.4(b), the flow rate increases as temperature increases from $215^{\circ} \mathrm{C}$ to $250^{\circ} \mathrm{C}$. Similar behavior for glass-fiber reinforced polypropylene was reported by Chiu et al. (1991), and is expected since higher temperature reduces viscosity. It can also be seen that the flow rate is more sensitive to the temperature at higher screw speeds. 


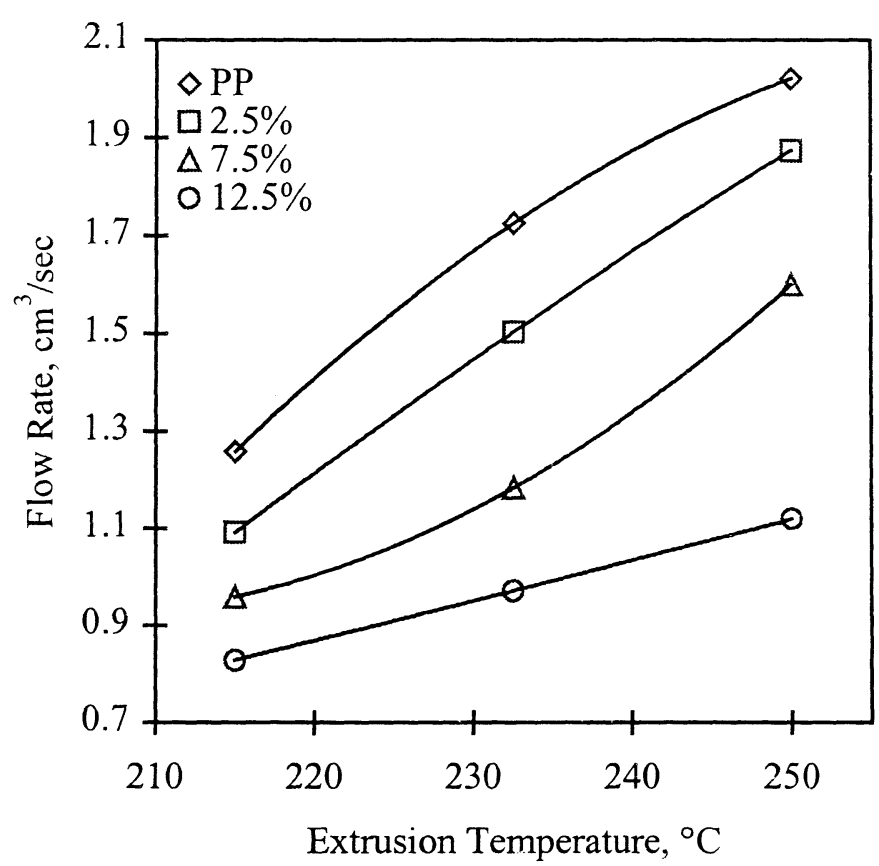

(a) Screw speed $=40$ RPM

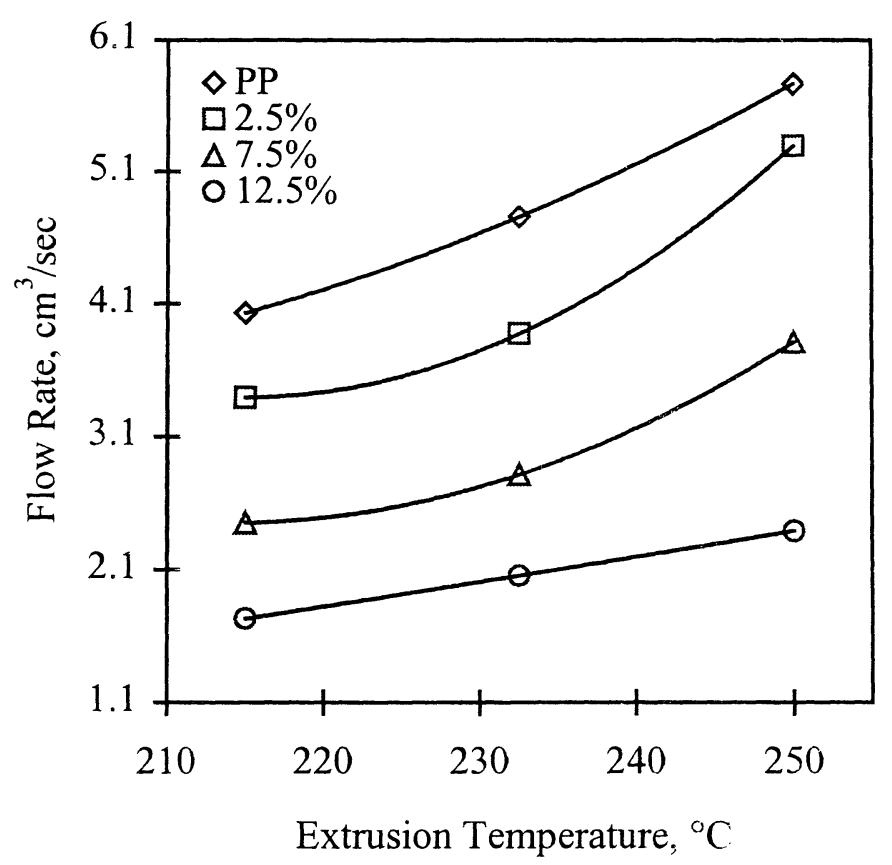

(b) Screw speed $=120$ RPM

Fig. 3.4. The dependence of flow rate on extrusion temperature at fixed screw rates. 
The relationships between extrusion temperature, extrusion pressure, and fiber content are shown in Fig. 3.5. As the temperature decreases from $250^{\circ} \mathrm{C}$ to $215^{\circ} \mathrm{C}$, the extrusion pressure increases; this behavior is seen at both screw speeds of 40 and 120 RPM. At lower fiber concentrations the pressure curve is linear as a function of temperature; at the highest fiber concentration of $12.5 \%$ by volume the pressure decreases in a nonlinear fashion. This indicates that the melt flow behavior is strongly temperature dependent at higher fiber concentrations. The increase in extrusion pressure can be caused by an increase in viscosity by the addition of VGCF. However, rheological studies have shown that viscosity of similar thermoplastics may or may not increase with fiber content. Laun (1984) has shown that glass fibers increased the viscosity of polypropylene, HDPE, LDPE, and polyamide. However, other studies (Caldeira et al., 1998; Carneiro et al., 1998) involving VGCF in polycarbonate matrices have showed that shear viscosity usually decreases with higher fiber content; this effect may be reversed at low shear rates for higher fiber content. Therefore, rheological tests of VGCF in polypropylene are planned as part of future experiments. 


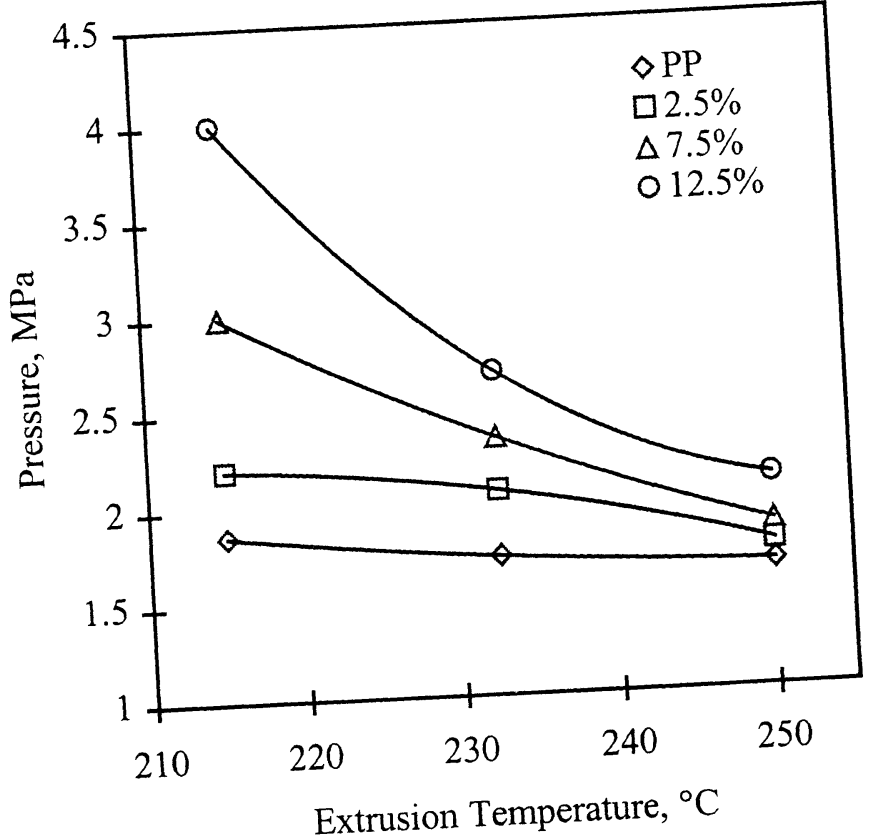

(a) Screw speed $=40$ RPM

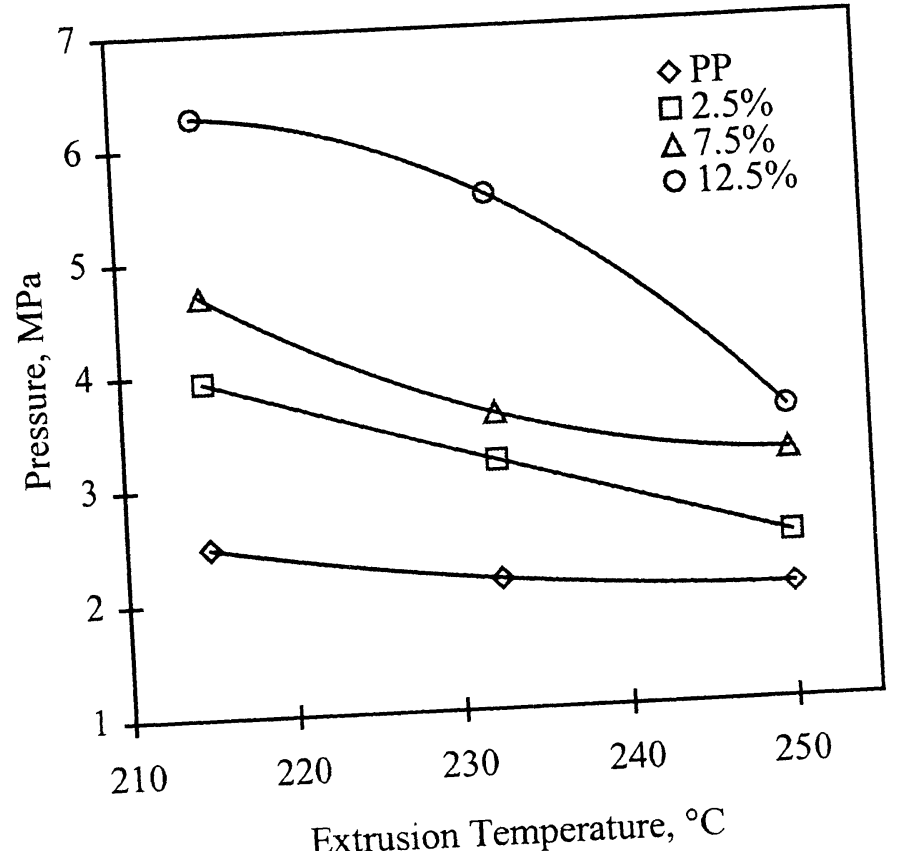

(b) Screw speed $=120$ RPM

Fig. 3.5. The dependence of pressure on extrusion temperature at fixed screw rates. 
Figure 3.6 shows the effect of the screw speed and fiber content on the flow rate and the extrusion pressure at a constant extrusion temperature of $215^{\circ} \mathrm{C}$. Both the flow rate and pressure increases as screw speeds increase from 40 to 120 RPM. As the fiber content increases, the flow rate decreases and the pressure increases. As discussed earlier, the flow rate should be linearly dependent on the screw speed since feed rate was kept proportional to screw speed. This is verified in Fig. 3.6(a). However, the extrusion pressure becomes strongly nonlinear with screw speed at higher fiber fractions (Fig. $3.6(b))$.

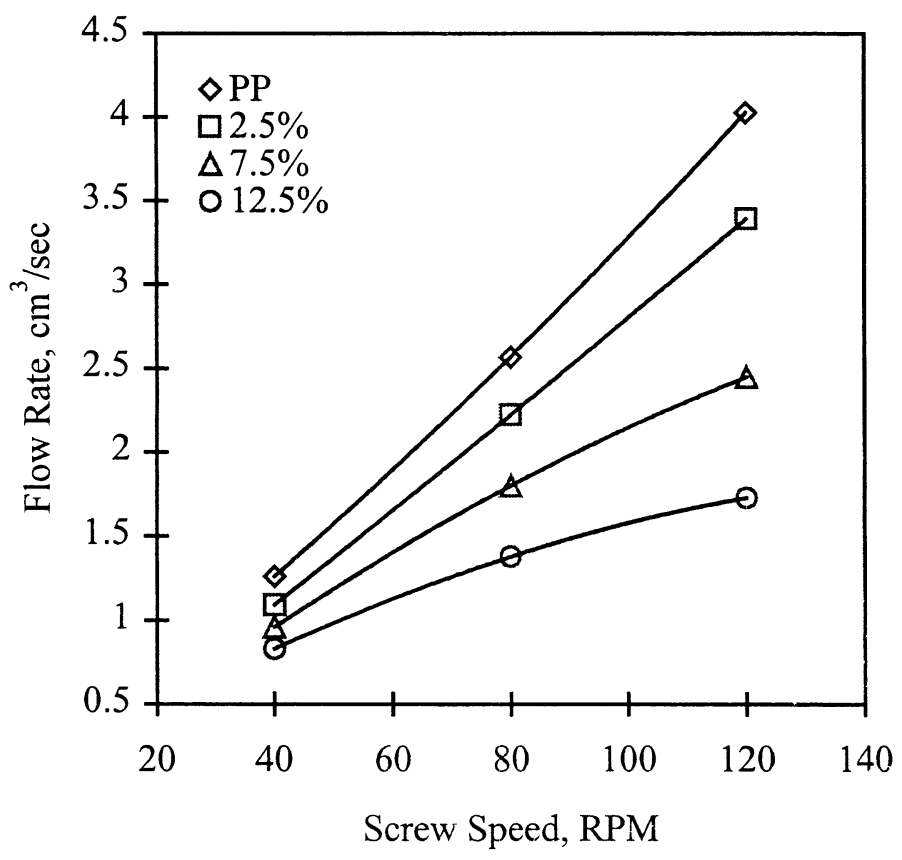

(a) Flow rate versus screw speed 


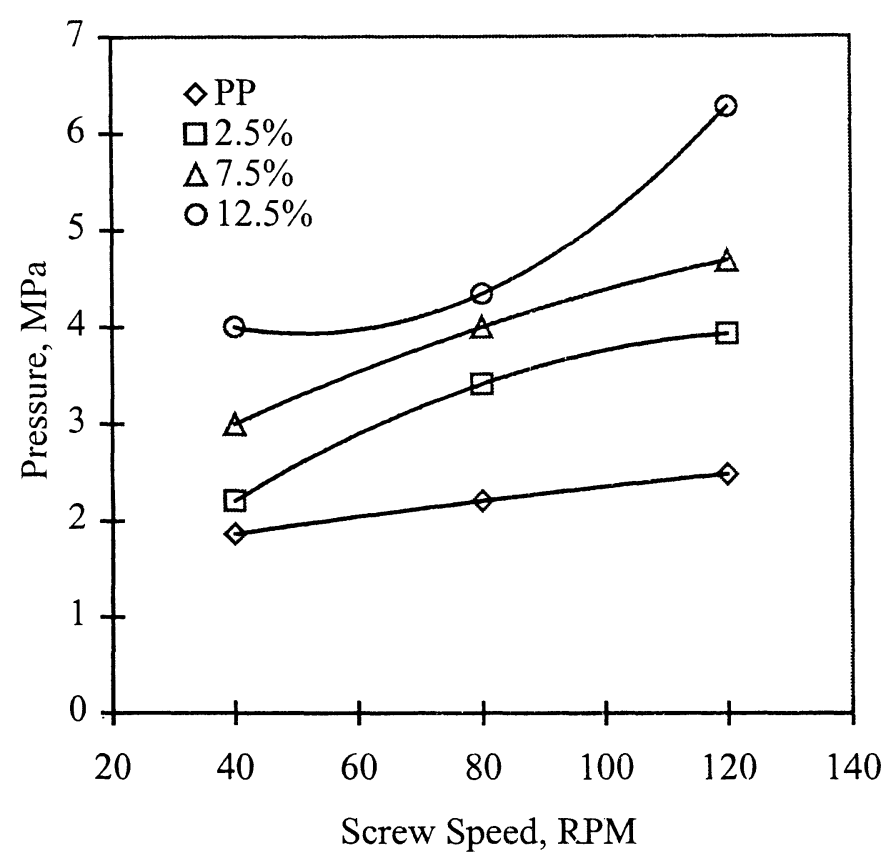

(b) Pressure versus screw speed

Fig. 3.6. The dependence of flow rate and pressure on the screw speed at a constant extrusion temperature of $215^{\circ} \mathrm{C}$.

\subsection{Fiber Length Characterization}

For optimal physical properties, the reinforcing fibers of the composite material must achieve a critical length. VGCF is microscopic in size, irregular in shape, and entangled within itself. During composite processing the fibers are typically shortened due to shearing or other mechanical forces. A typical process may include ball-milling of the fiber (Tibbetts and McHugh, 1999). At the end of a typical ball-milling process for 1 minute the fibers still have a good aspect ratio as shown in Fig. 3.7. 


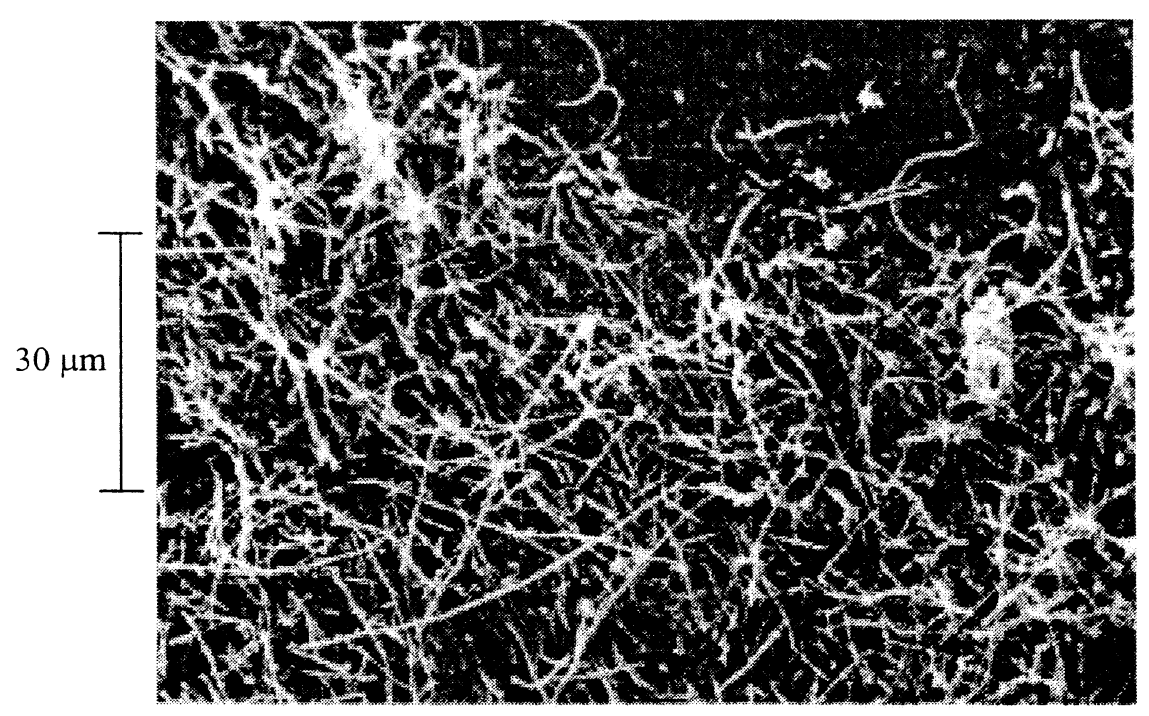

Fig. 3.7. SEM of VGCF after ball-milling for 1 minute (Tibbetts and McHugh, 1999).

An important issue is whether the shearing forces produced by the extruder screws in the high viscosity fiber/polymer mix would degrade or destroy the fibers. To address this issue, a scanning electron microscopy (SEM) analysis was performed on each composite sample extruded at the various conditions. Since the fibers are grown with lengths ranging between $20-80$ microns, it is hard to quantify the actual amount of fiber breakage that occurs during extrusion. However, a qualitative analysis can be made by examining the fibers in the composites after processing. This was accomplished by heating the composite samples in an oven at approximately $400^{\circ} \mathrm{C}$ for 2 hours in argon. This removed the matrix material from the specimens. The length and integrity of the remaining fibers were then observed under a SEM. 
Figure 3.8 shows an SEM photograph of a $7.5 \%$ VGCF reinforced sample extruded at $215^{\circ} \mathrm{C}$ with a screw speed of $80 \mathrm{RPM}$, and then processed to remove the matrix material as described above. It can be seen that the fibers still have a good aspect ratio and are comparable to the fibers shown in Fig. 3.7. The result is typical of all the specimens analyzed in this study.

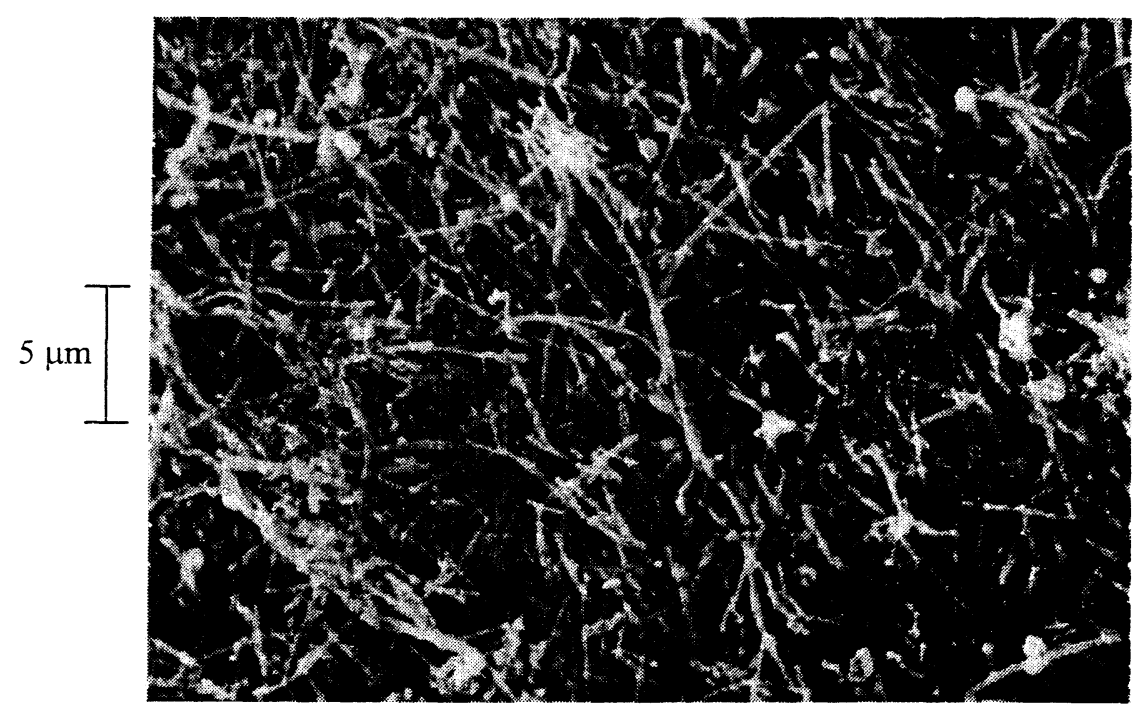

Fig. 3.8. SEM of VGCF after the removal of the matrix from the extruded composite.

A more accurate approach to determine fiber length was done by performing transmission electron microscopy (TEM) on the composite samples. After removing the polypropylene matrix from the samples as previously described, a small amount of fibers was then dispersed in an alcohol suspension and viewed on a 3000 mesh grid. The fibers were then viewed under the TEM to determine the fiber length. From the micrographs in 
the regions where the fibers were dispersed the average fiber length was found by averaging the lengths of several fibers and was found to be approximately 10.42 microns. It will be shown later that the tensile strength is decreased at higher screw speeds, which indicates that fiber length may be reduced due to higher shear. However, due to the entanglement of the fibers, tests on fiber length were not conclusive enough to accurately quantify the change in fiber length at different processing conditions.

\subsection{Mechanical Properties}

To evaluate the mechanical properties of the VGCF composites, tensile rest samples were prepared by cutting and placing the strands in a unidirectional manner into a $10 \mathrm{~cm}$ by $20 \mathrm{~cm}$ mold with a temperature of approximately $230^{\circ} \mathrm{C}$. The strands vere then slowly hot-pressed into a 2 to $3 \mathrm{~mm}$ thick sheet. The mold was then inmiediateiy submerged into a cold water bath to help prevent crystallization during solidification. Entrapment of air pockets or voids was a problem during initial trial rins, especially in the areas at the center of the mold. The molding of specimens with higher fiber contersts ( $>10 \%$ fiber volume fraction) was challenging because the fibers increased the viscosity of the mixture, thus making it more difficult for the air pockets to escape. In order to alleviate this problem, the molding process was performed in an evacuated chamber.

Tensile test specimens were formulated with the PR-21-AG fiber containing volume fractions ranging from $2.5 \%$ to $12.5 \%$. To prepare tensile test specimens, rectangular strips were then sawed from the composite sheets along the extrusion direction. The strips were then machined in a router fixture conforming to ASTM D638 
Type II specifications. The ultimate tensile strength and modulus was then measured and recorded by a Tinius Olsen bench-top testing machine equipped with a Keithley data acquisition system with an extension rate of $4 \mathrm{~mm} / \mathrm{min}$.

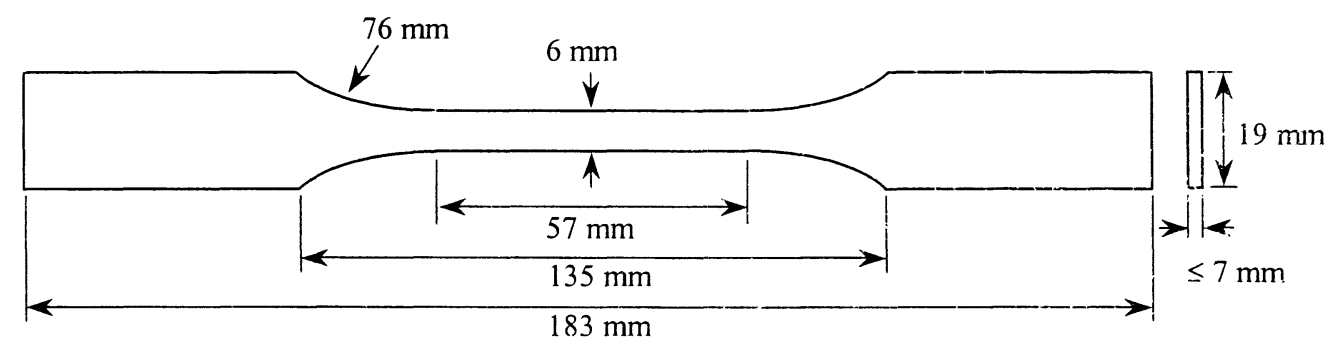

Fig. 3.9. Specifications of ASTM D638 Type II tensile specimen.

The ultimate tensile strength and modulus with respect to extrusion conditions are shown in the following figures. Figures $3.10(a)$ and $3.10(\mathrm{~b})$ present the terisile strength and modulus, respectively, of the extrudate while processed at a constant screw speed and various temperatures. The strength and stiffness behavior at constant ternperature and various screw speeds is then compared in Figs. 3.11(a) and 3.11(b). It is apparent from the graphs that both the strength and stiffness of the composite materials are increased from the matrix strength as the percentage of fiber increases. However, the dependence of the strength and stiffness on the extrusion temperature may depend on a number of factors including the polymer behavior at different temperatures. For example, the tensile strength decreased from $215^{\circ} \mathrm{C}$ to $232^{\circ} \mathrm{C}$ and then increased at $250^{\circ} \mathrm{C}$. On the other hand, the tensile modulus showed a reverse trend, increasing to a maximum value at $232^{\circ} \mathrm{C}$. It is possible that the fiber/polymer interaction is optimized at $232^{\circ} \mathrm{C}$, which would produce 
a stiff composite that is brittle and therefore having lower tensile strength. It should be noted that certain varieties of VGCF do not produce significant improvements in tensile strength at higher fiber fractions (Tibbetts, 2000). This can be observed in Fig. 11(a), where there is little variation between the strength of $7.5 \%$ and $12.5 \%$ VGCF samples. An exception to the general trend is the modulus value for $7.5 \%$ fiber, which goes down significantly with screw speed. The overall trend is a small decrease in tensile strength (less than 10\%) and modulus of the composites (not pure polypropylene) at higher screw speeds. This may be due to some fiber degradation at the higher shear rates. It has been shown by Dospisil et al. (1994) that glass fibers decrease significantly in length by single screw extrusion. However, VGCF is very short and because the fibers are crooked and entangled, it was not possible to experimentally determine small changes in fiber iength in the composites produced due to variation of extrusion parameters in our experiments.

Experimental results showed that there was less than $5 \%$ strength difference between the composites produced from the first compounding stage followed by a second extrusion stage and the composites produced by using a single stage extrusion process. It appears that the fibers are broken when the fiber clumps are torn apart by the shearing forces in the extruder during the compounding stage. However, once the fiber clumps are broken apart, the fiber lengths may not change significantly during subsequent processing. 


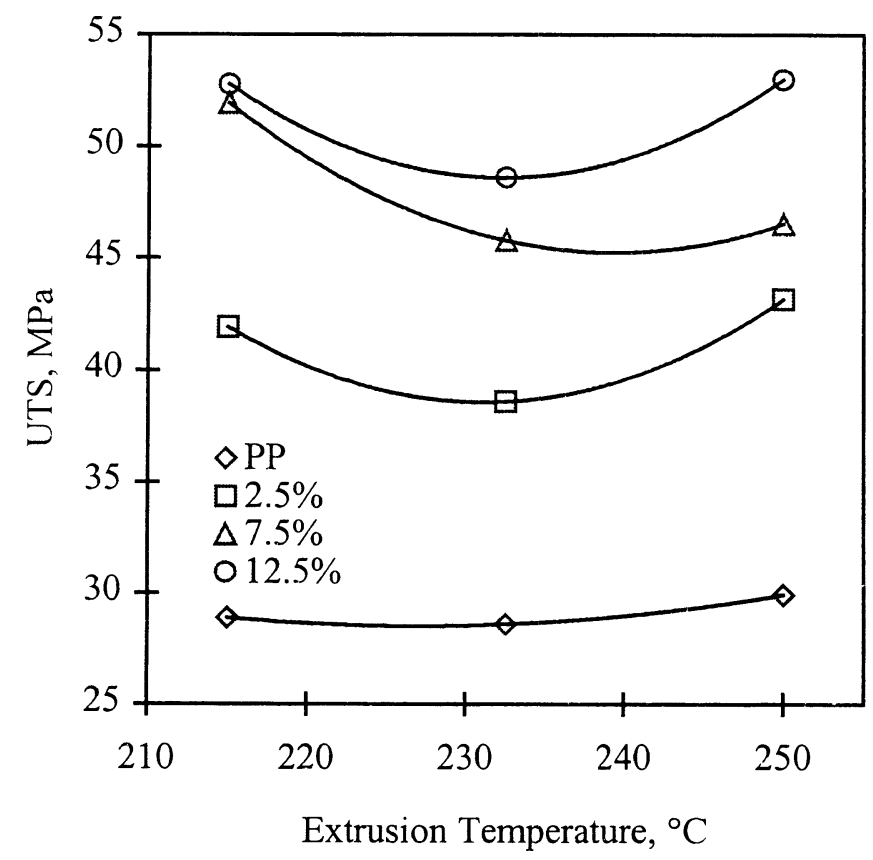

(a) Ultimate tensile strength versus extrusion temperature

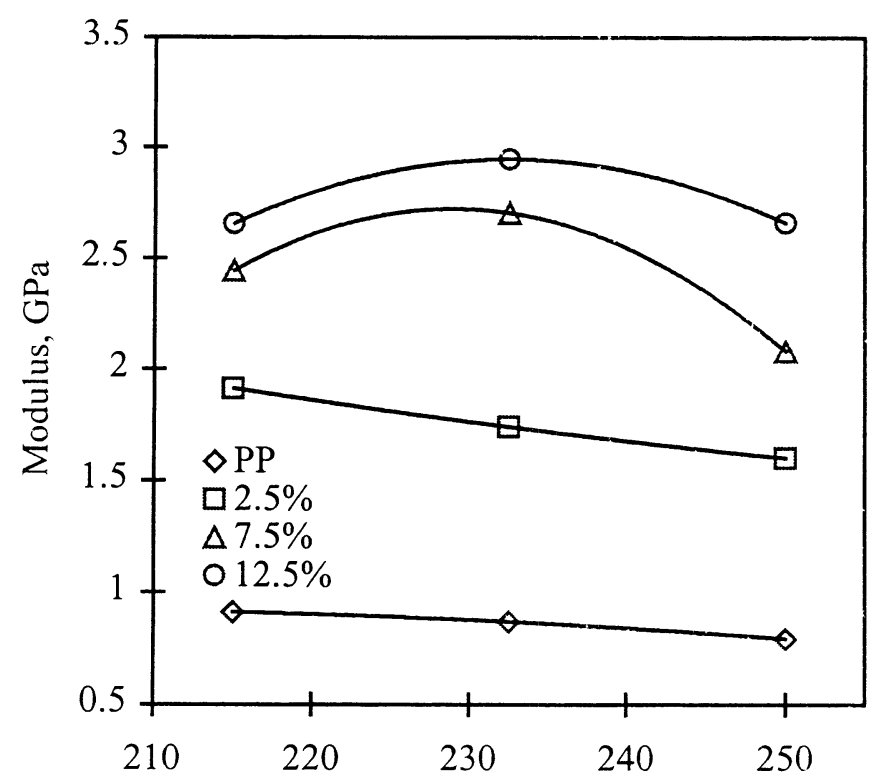

Extrusion Temperature, ${ }^{\circ} \mathrm{C}$

(b) Tensile modulus versus extrusion temperature.

Fig. 3.10. Variation of ultimate tensile strength and modulus versus extrusion temperature at a constant screw speed of 40 RPM. 


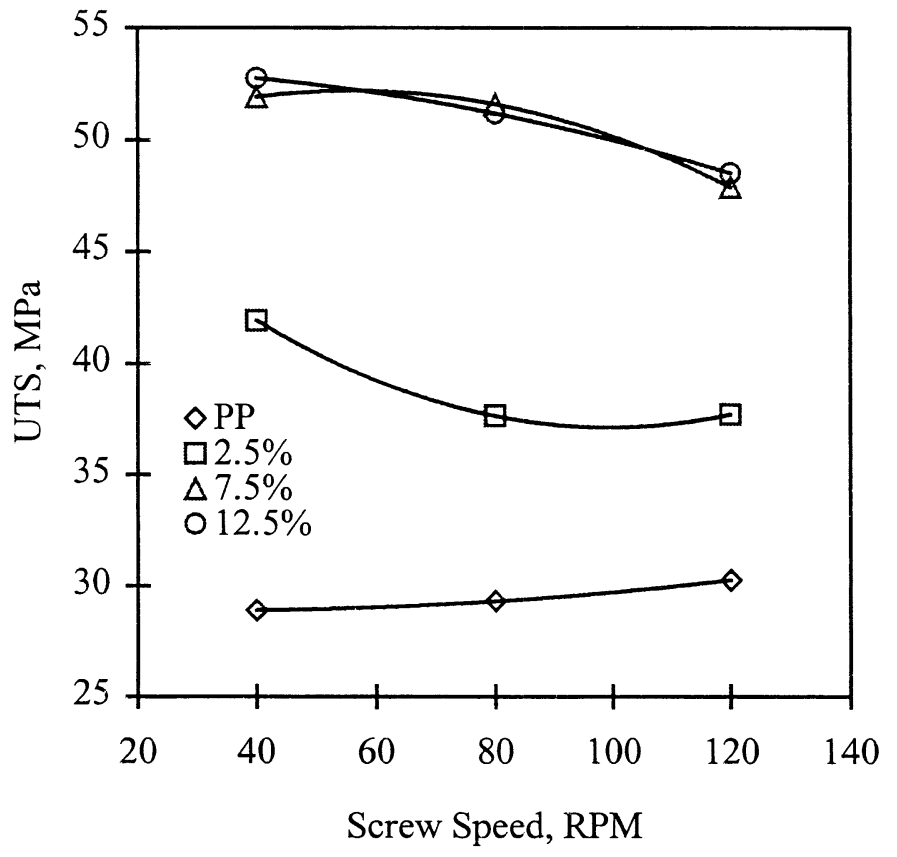

(a) Ultimate tensile strength versus extrusion temperature

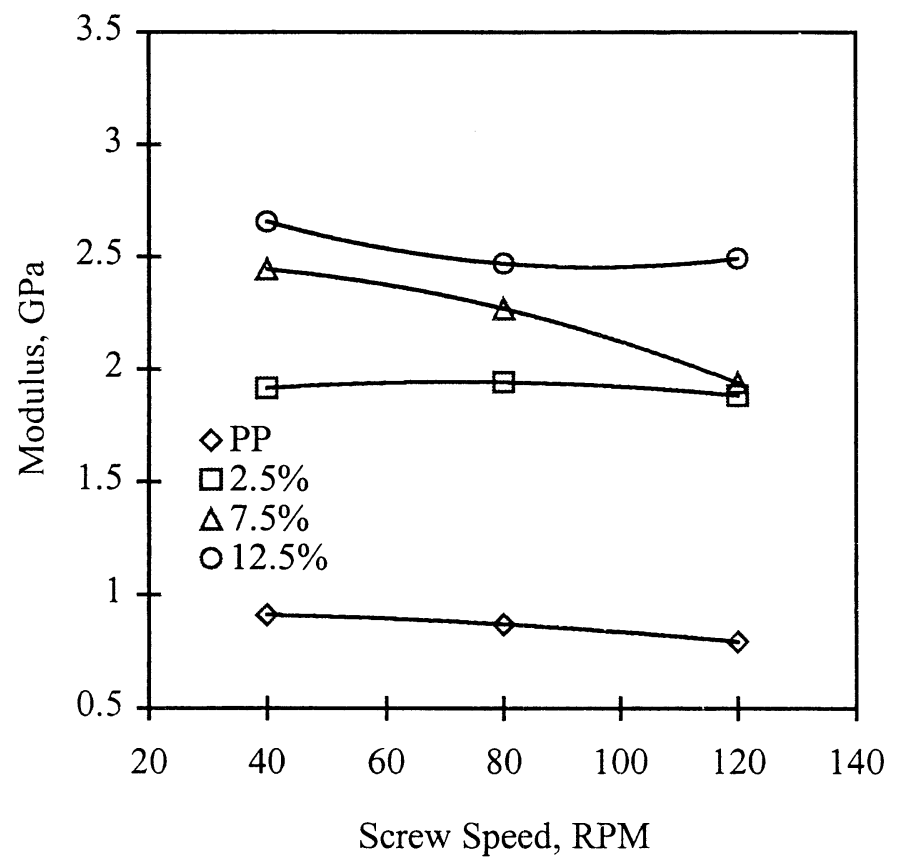

(b) Tensile modulus versus extrusion temperature.

Fig. 3.11. Variation of ultimate tensile strength and modulus versus screw speed at a constant extrusion temperature of $215^{\circ} \mathrm{C}$. 
The previous results were analyzed to determine the standard deviation in the data. For example, for the case of $12.5 \%$ vol. VGCF, the standard deviation was $4.1 \%$ of the ultimate strength value and $3.7 \%$ of the modulus value. This is typical of most samples. An exception was the $7 \%$ vol. VGCF in which the standard deviation was $5.8 \%$ of the ultimate strength value and $1.6 \%$ of the modulus value.

To make a comparison between the mechanical property results obtained in this work and those of other studies, the data taken with the base screw speed of 40 RPM and an extrusion temperature of $215^{\circ} \mathrm{C}$ was chosen. These results are compared in Figs. 3.12 and 3.13 with experimental results from injection-molded samples reported by Van Hattum et al. (1999) and Tibbetts and McHugh (1999).

The experimental data from Tibbetts and McHugh is for 'ball-milled best shot' samples, which are very similar to PR-21-AG (Glasgow, 2000) used for this study. The tensile strength of the samples is higher than what is reported in studies by Van Hatturn et al. (1999) and Tibbetts and McHugh (1999). This indicates that the extrusion process in our experiments appear to have produced significant fiber orientation along the extrusion direction. Tibbetts and McHugh (1999) have also reported orientation effects in their composite samples. The tensile modulus increases significantly over the value for the unreinforced material. The tensile modulus values are intermediate between the data of Van Hattum et al. (1999) and Tibbetts and McHugh (1999). 


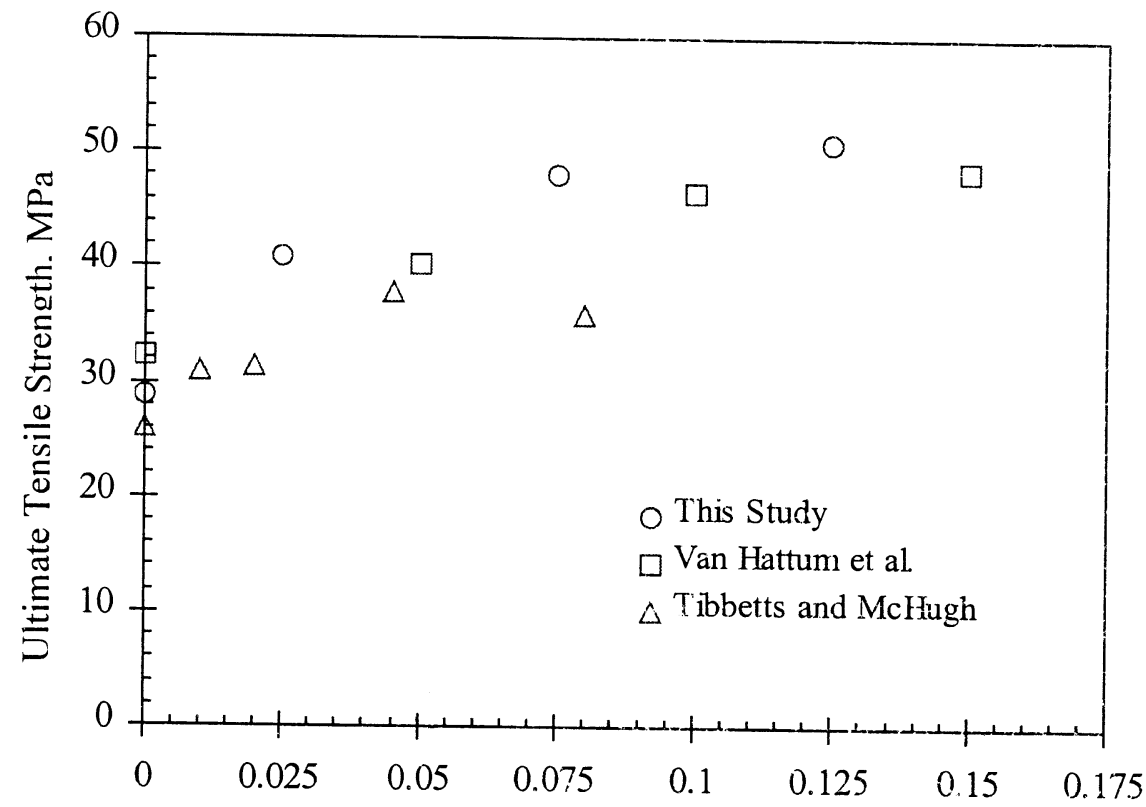

Fiber Volume Fraction

Fig. 3.12. Experimental results for tensile strength of composite samples at various fiber volume fractions.

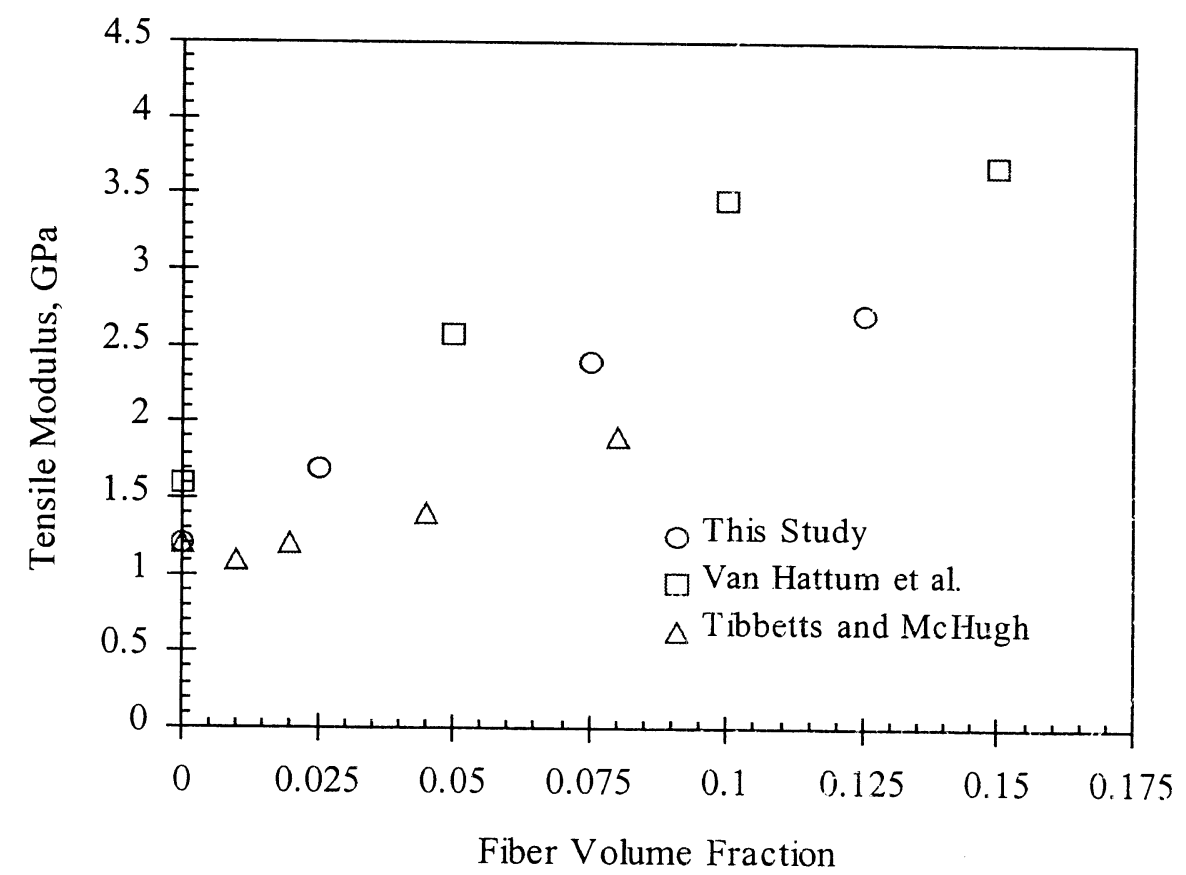

Fig. 3.13. Experimental results for tensile modulus of composite samples at various fiber volume fractions. 


\subsection{Void Content}

Void content can adversely affect the bulk properties of a composite. Therefore, to study this relationship, the ASTM D2734 Method A guideline was used to determine void percentage of the VGCF reinforced thermoplastic specimens at various extrusion conditions. In this method the theoretical density is first calculated from the known specific gravity of the fiber and polypropylene which are 2.0 (ASI, 1999) and 0.9 (Montell, 1999), respectively. The theoretical value was then compared to the experimental density of the specimen to find the void content. This is shown in Fig. 3.14 where the void percentage at different fiber contents and extrusion conditions were determined.

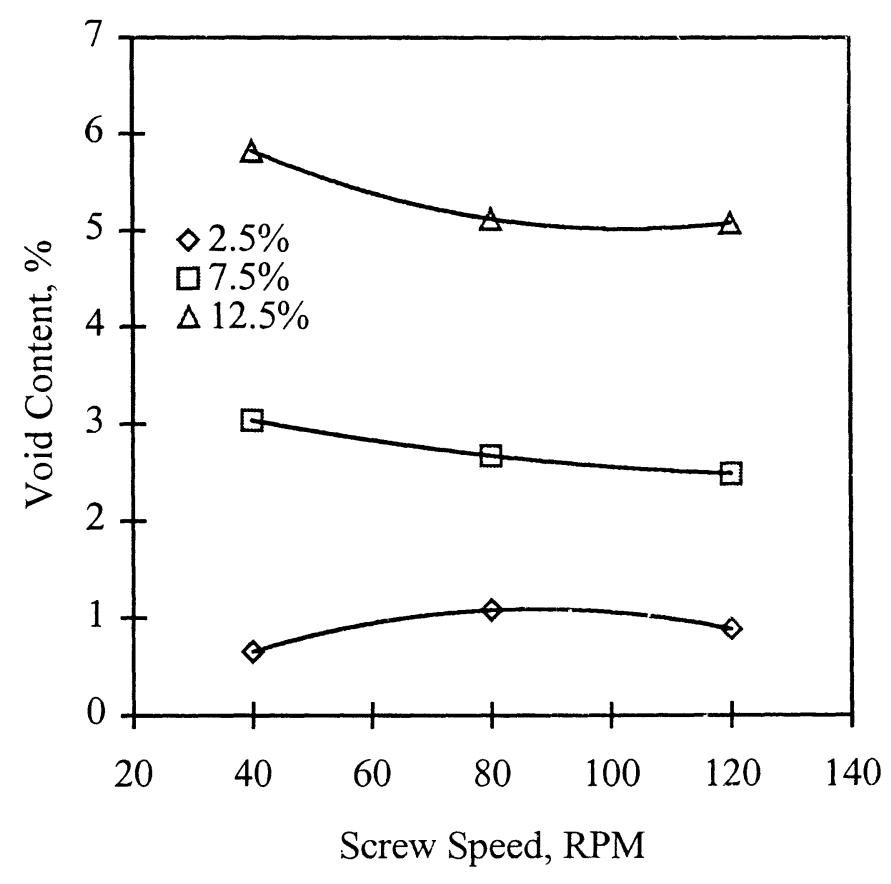

(a) Void content versus screw speed with extrusion temperature at $215^{\circ} \mathrm{C}$. 


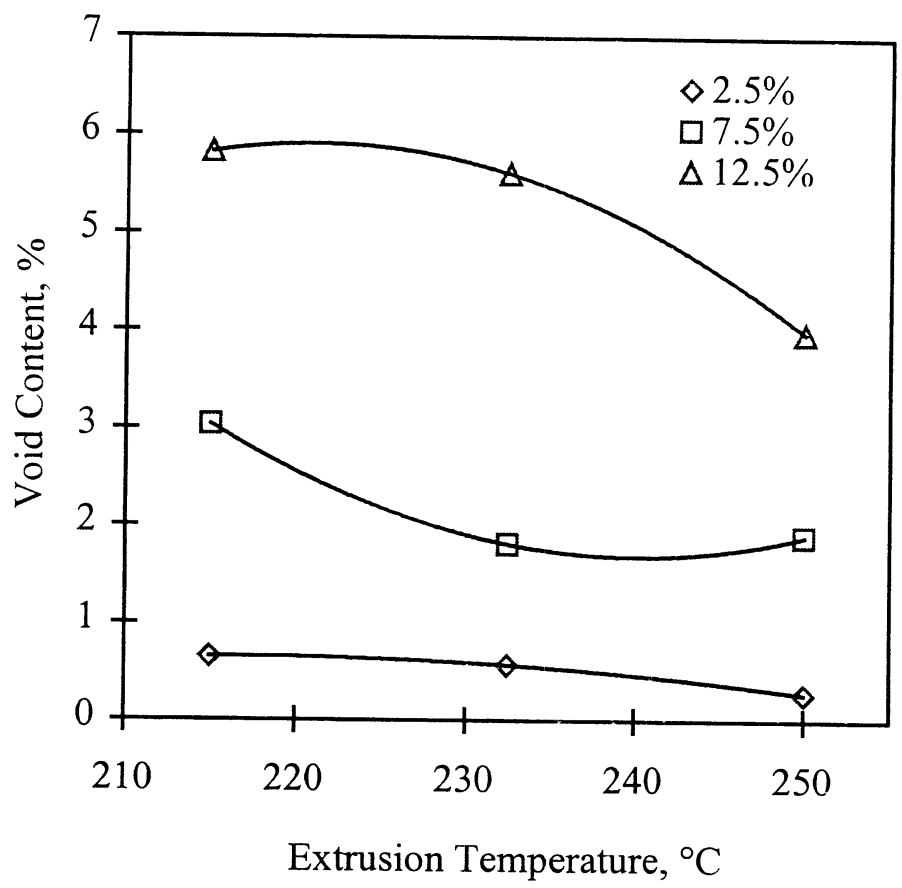

(b) Void content versus extrusion temperature with screw speed at 40 RPM.

Fig. 3.14. Variation of void content versus screw speed and temperature.

The percentage of voids increases as fiber content increases. This is most likely due to the fact that it is harder for the polymer melt to infiltrate and wet large amounts of fiber. Since VGCF is generated in an airborne configuration driven by a gas flow, the fibers become tangled when two or more intersect in the gas stream. This results in a mass or clump of microscopic entangled fibers as shown in Fig 3.15. 


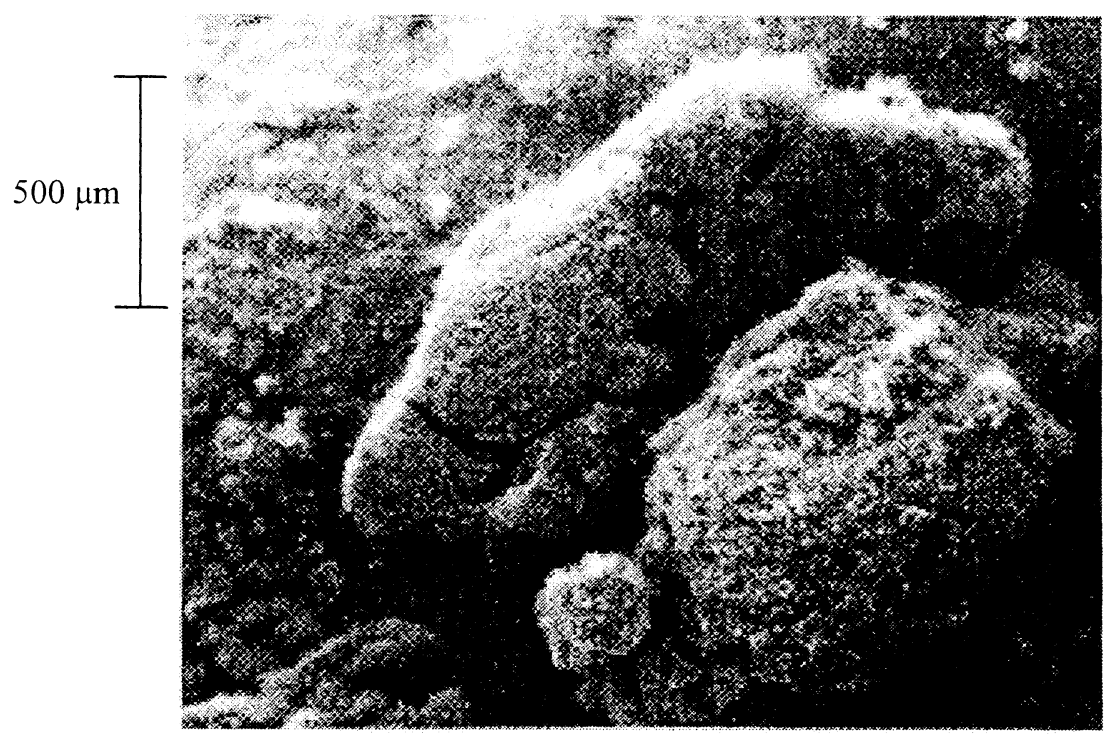

Fig 3.15. SEM of clumps of VGCF (Tibbetts and McHugh, 1999).

It was suggested by Tibbetts and McHugh (1999) that the infiltration of the polymer matrix into these fiber clumps can be increased by high shear mixing, using less viscous polymers, and infiltrating at higher pressures for longer periods of time. This is seen to be the case as the void content decreases when the extrusion pressure goes up with screw speed (Fig. 3.5), and the polymer viscosity goes down with temperature (Fig. $3.4)$.

Tibbetts and McHugh (1999) also suggested ball-milling the raw VGCF to reduce the size of the fiber clumps to increase polymer infiltration. This technique proved successful by improving infiltration by both nylon and polypropylene matrices, thus improving material properties in every aspect. It was also shown by Mortensen et al. 
(1989) that the pressure required to infiltrate a porous preform or clump of VGCF is the sum of the pressure required to overcome capillary effects, and the pressure required to overcome viscous drag. Therefore, infiltration is promoted by processing the VGCF/polymer blend at higher pressures. 'This is illustrated in Fig. 3.16 where the void content decreases at higher extrusion pressures, thus suggesting greater infiltration.

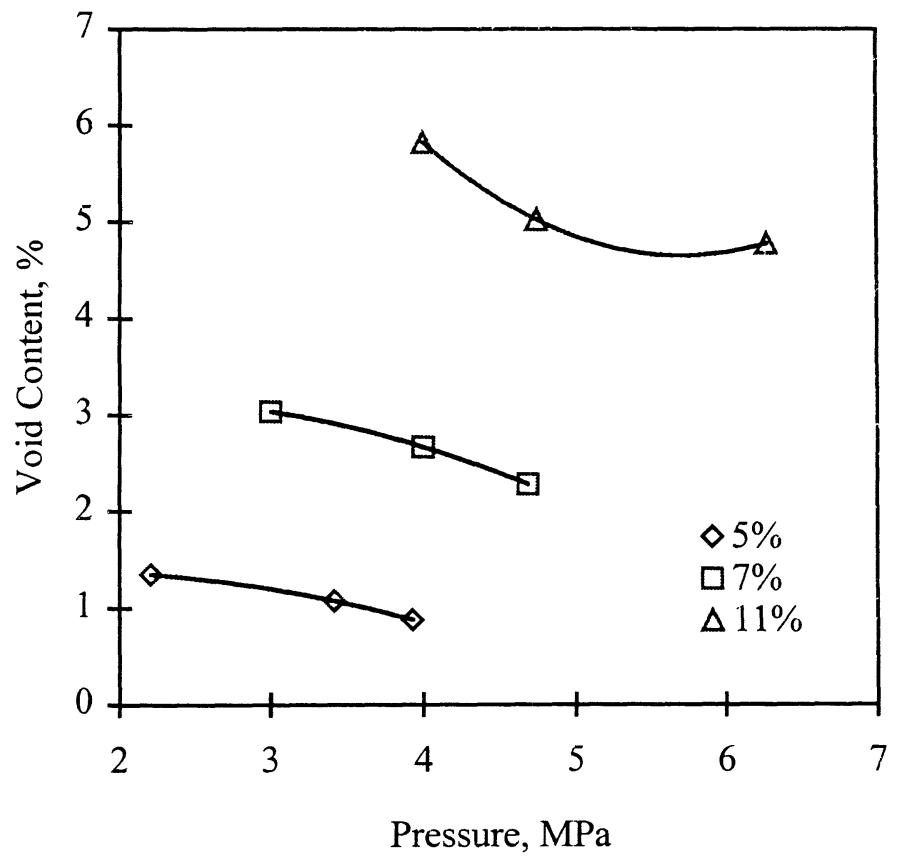

Fig. 3.16. Void content versus extrusion pressure of VGCF reinforced polypropylene.

\subsection{Effect of VGCF on Polymer Flow}

Experimental results show that the extrusion process is strongly dependent on the fiber content of the composite. The addition of VGCF provided an increase in the extrusion pressure. This seems to indicate that the viscosity of polypropylene is raised by 
addition of VGCF. The shear rates in these experiments are on the order of 100 to 1000 per second. The increase in extrusion pressure can be caused by an increase in viscosity by the addition of VGCF. However, rheological studies have shown that viscosity of other thermoplastics may or may not increase with fiber content. Laun (1984) has shown that glass fibers increased the viscosity of polypropylene, HDPE, LDPE, and polyamide. However, other studies (Caldeira et al., 1998; Carneiro et al., 1998) involving VGCF in polycarbonate matrices have showed that shear viscosity at high shear rates decreases with higher fiber content; this effect may be reversed at low shear rates for higher fiber content. Therefore, the effect of VGCF on the viscosity of the VGCF/polypropylene mixture needs to be verified by rheological measurements.

The extrusion temperature ranged from $215^{\circ} \mathrm{C}$ to $250^{\circ} \mathrm{C}$. This is significantly higher than the approximate minimum processing temperature of $200^{\circ} \mathrm{C}$ for polypropylene. At the lower temperatures, the viscosity is expected to be higher; consequently, the extrusion pressure was as high as $6.2 \mathrm{MPa}$. The tensile strength results indicate that the flow in the extrusion process tends to align the fibers in the composite. 


\section{CHAPTER 4}

\section{PROCESSING AND CHARACTERIZATION OF ALIGNED VGCF REINFORCED POLYPROPYLENE}

\subsection{Introduction}

The properties of composites are not only functions of the fiber and polymeric matrix materials, but also the state of fiber orientation. The work presented in this chapter will introduce a method to produce an improved anisotropic composite by alignment of the nanometer-size PR-21-PS fiber. The degree of fiber alignment will be measured using an x-ray diffraction technique and aligriment results for composite strands, sheets, and cubes are presented.

\subsection{X-ray Diffraction}

$\mathrm{X}$-ray diffraction is an analytical technique that uses a beam of $\mathrm{x}$-rays to probe repeating planes of atoms. The reflection of x-rays off of repeating planes of atoms creates a series of spots called a diffraction pattern. Laue patterns are the easiest x-ray diffraction patterns that can be obtained and are very useful for measuring the orientation of single crystals. Laue patterns consist of individual spots for single crystals and rings for randomly oriented powders. Oriented fibers produce Laue patterns that are between those extremes and are seen as arcs. The intensity distribution around the arcs is related to the orientation of the diffracting planes (Roe and Krigbaum, 1964; Alexander, 1969). This orientation information has been used in carbon fibers to determine the approximate degree of alignment of the graphene planes in those fibers with respect to the fiber axis 
(Ruland, 1968). The intensity distribution is measured by obtaining the intensity at a fixed Bragg angle (or $2 \theta$ ) as a function of the angle relative to the fiber axis direction (azimuthal angle). This is the same as measuring the intensity around the arc of the Laue pattern.

The scattered $\mathrm{x}$-ray pattern is obtained using a flat image plate held perpendicular to the main beam after it passes through the sample (Fig. 4.1). This is known as the transmission Laue method. The resulting digital $x-y$ data of the pattern is similar to a Statton camera flat-film pattern or a monochromatic, polycrystalline Laue pattern. The grayscale values $\left(V_{g}\right)$ making up the $\mathrm{z}$-axis of the digital pattern are related to the intensity $(I)$ of the $\mathrm{x}$-ray scatter by the following equation

$$
I=10^{R \cdot V_{g} / 65535}
$$

where $R$ equals the intensity decade range of the image plate as it was read (usually 4) and 65535 represents the maximum grayscale value. The intensity can then range for 1 to 10,000 counts as the grayscale value ranges from 0 to 65535 . The absolute intensity is not important since it is dependent on the exposure times and those times were adjusted to maintain the maximum observed grayscale level below 65535 (usually about 60 seconds). 


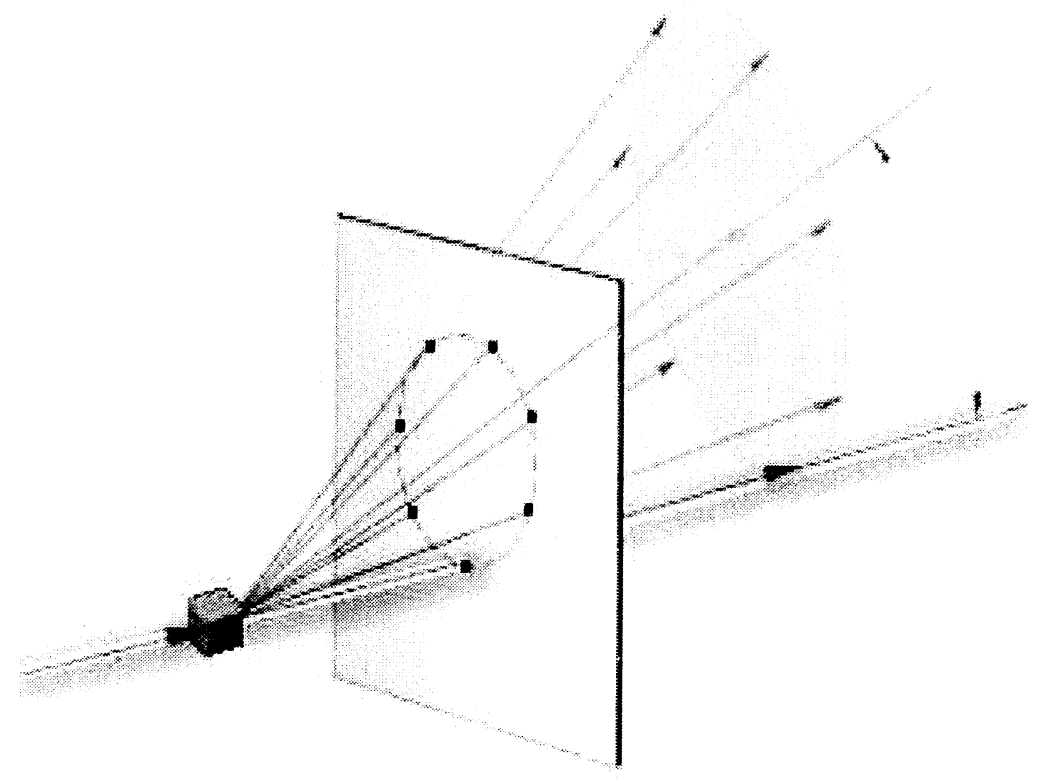

Fig. 4.1. Transmission Laue method where the film is place behind the sample to record the beams which are transmitted through the sample.

Bragg diffraction data on a sample can be obtained by taking the intensity values along a line of pixels passing through the center of the pattern. Bragg scans at different angles relative to the fiber axis can be obtained if desired or the line scans at all angles averaged to simulate a powder diffraction pattern. Azimuthal data can be obtained by reading the intensity around a circle of pixels. The center of the circie must correspond to the center of the pattern and the radius of the circle must correspond to the distance from the pattern center for the Bragg angle of the desired crystalline reflection. Fiber diffraction patterns are always symmetric right to left and up and down. Theoretically only one fourth of the pattern need be collected but in practice the entire pattern is 
collected on film or an image plate and the symmetry used to locate the exact center of the pattern.

Diffracting planes must still satisfy the Bragg condition to be visible and therefore only equatorial planes (such as the $(0,0,2)$ graphitic plan in carbon fibers) are always visible. Some meridional and off-axis planes can also be visible if they happen to lie at the Bragg incident angle relative to fiber axis so as to satisfy the Bragg condition. In our experiment, none of the non-equatorial reflections were of interest nor were they examined.

For each of the samples examined, the center of the patterns was determined by finding the location equidistance from each the polypropylene and graphitic fiber diffraction rings both along an $\mathrm{x}$-direction and along a y-direction. Usually only one or two adjustments of the starting center were required. The distance from the pattern center to the graphitic $(0,0,2)$ reflection (in pixels) was usually determined during the course of locating the pattern center. This value was then used as the radius of the azimuthal circle. The intensity of the pixels was then read at that radius about the center at one degree increments around the pattern.

Figure 4.2 shows a fiber flat-film Laue pattern with the $(0,0,2)$ arcs of a carbon fiber. Azimuthal data is collected by measuring the intensity around the arc as a function of angle. The full-width and half maximum is used as a measure of the graphene planes' alignment. In this figure, only part of the intensity versus azimuthal angle is shown but often the entire $360^{\circ}$ is measured. 


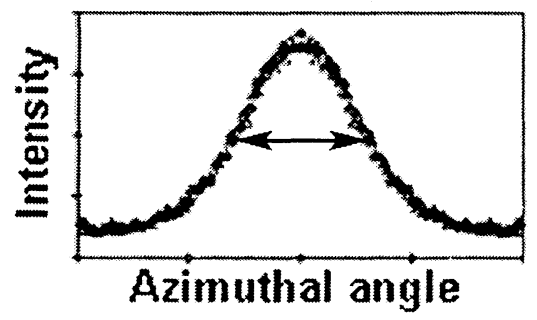

Fig. 4.2. A flat-film Laue pattern with the $(0,0,2)$ arcs of a carbon fiber.

It is often necessary to go around the figure more than $360^{\circ}$ to get a better fitting of peaks that happen to fall at or near 0 and $360^{\circ}$. Starting at a different angle would accomplish the same result but it is easier to always start at the same place and just keep going.

An example of a composite sample with aligned fiber reinforcement is shown in Fig. 4.3. It is seen that the fiber diffraction is less than the polypropylene (polypropylene has darker rings) but the $(0,0,2)$ of graphite is still visible. The fact that the diffraction 
comes out at different arc radii (Bragg angle) helps to both distinguish and identify the diffraction planes.

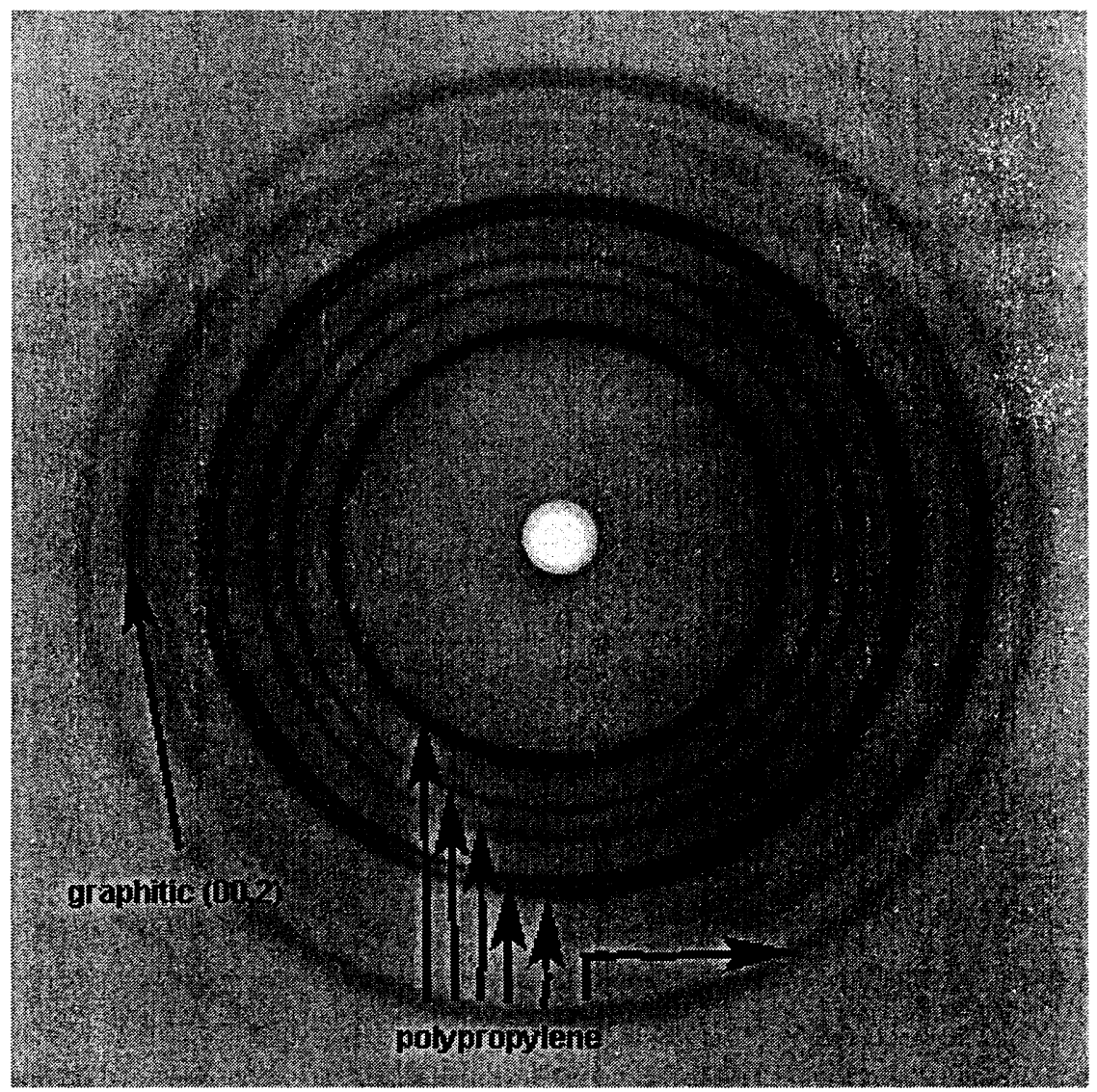

Fig. 4.3. An example of the diffraction planes of a composite sample with aligned fiber reinforcement.

Sophisticated computer software is used to locate the arcs and rings and then measure the intensity as a function of azimuthal angle and store the data to a file. By looking at the inner dark ring of polypropylene one should be able to notice the darkening 
or thickening of the ring at the top and bottom of this figure. This is an indication that the polypropylene is also being aligned in the composite sample.

The data file was then analyzed using PeakFit ${ }^{\top M} 4.0$ for Windows ${ }^{\circledR}$ by SPSS, Inc. Since the composite samples were held vertical, the fiber $(0,0,2)$ peaks occur near an azimuthal angle of zero degrees. The scans were curve fit from -90 to $+90^{\circ}$ using a straight baseline and a single gaussian peak. The automated residuals method of fitting was used which adjusts the location, height, and width of the peak and the baseline coefficients to minimize the sum of residuals (or error) between the experimental points and the total fit curve. The baseline coefficients are dominated by the intensity at the each end of the curve and the peak parameters determined by the points around the maximum intensity. The program outputs the fitting parameters as well as the peak area and full-width at half-maximum. This latter value is the commonly used " $Z$ " parameter for graphitic misalignment in carbon fibers.

Some of the $\mathrm{x}$-ray diffraction data was collected at Beam line $\mathrm{X} 27 \mathrm{C}$ at the National Synchrotron Light Source, Brookhaven National Laboratory. This Beam line used double crystal monochromated synchrotron radiation at a wavelength of $0.1307 \mathrm{~nm}$ incident to the sample with flat-film Laue data collected by an image plate. Other x-ray diffraction studies were carried out using a Huber 4-circle x-ray diffractometer in symmetric transmission with an incident beam crystal monochromated $\mathrm{CuK} \alpha$ radiation $(\lambda=0.15418 \mathrm{~nm})$ from a Rigaku RU-200 rotating anode generator at a power of $45 \mathrm{kV}$ and $70 \mathrm{~mA}$. 


\subsection{Flow-Induced Alignment of VGCF}

The procedure to produce an improved polymeric composite by alignment of nanometer-size VGCF in the polymer matrix follows the extrusion process described in Section 3.2 of the previous chapter. In order to generate a high degree of fiber alignment during this process, the extruder was equipped with a special die. The unique design of the die generates a high degree of flow-induced fiber alignment by taking advantage of two distinct flow regimes that affects fiber orientation. First, the die inlet initiates a converging flow pattern that tends to align the fibers along the streamline direction (extrusion ratio $\approx 48$ ). This flow pattern is then transformed into shear flow while entering a $1.25 \mathrm{~cm}$ long annular portion of the $2 \mathrm{~mm}$ diameter die (Fig. 4.4). The shear flow produces additional fiber alignment due to the varying shear rate along the boundary layer that orients the fibers in the direction of flow.

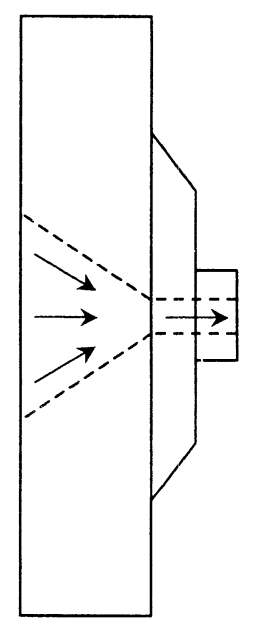

Fig. 4.4. Side view of extruder die 
Composite mixtures formulated with the PR-21-PS fiber at various volume fractions were extruded through the die configuration shown in Fig. 4.4 to produce a uniform diameter composite strand reinforced with aligned VGCF. By using x-ray diffraction, it is possible to measure the amount of fiber alignment along the axis of the composite strand. As previously described, the commonly used measure of carbon fiber alignment is the full-width of the $(0,0,2)$ graphitic plane azimuthal diffraction measured at one-half the maximum intensity. This measurement is designated as ' $Z$ ' and given in degrees. This measure represents the spread of the majority of graphene planes. This value is the full spread of the misalignment and should be thought of as a cone angle since the alignment is in three dimensions. The value should be halved if one wished to think of how far from the preferred axis that the fibers are misaligned.

When this measurement is made on a composite specimen, a measure of the alignment of the fibers can be obtained from the graphene alignment within the composite. Some of the spread in the azimuthal intensity is from misalignment of the graphene planes but the remainder is from the misalignment of the fibers within the strand. Therefore, a relative amount of alignment can be inferred from this measurement. Figures 4.5(a), 4.5(b), and 4.5(c) show the x-ray diffraction profiles of composite samples containing $2.5 \%, 7 \%$, and $11 \%$ VGCF by volume.

The intensity of the $\mathrm{x}$-ray diffraction at different angles indicates the distribution of fiber orientation in the composites. The specimens were tested so the 0 degree azimuthal angle corresponds to the preferred direction, and 90 degrees corresponds to the transverse direction of the composite materials. If the fibers are aligned, the intensity of 
the diffraction pattern at high angles should be very low, whereas the intensity in the preferred direction ( 0 degrees) should be relatively high. The leveled intensity at \pm 90 degrees is the background intensity. This can be seen in Figs. 4.5(a), 4.5(b) and 4.5(c), where the fibers are oriented $\pm 23.7, \pm 28.15$ and \pm 30.0 degrees along the preferred direction for the $2.5 \%, 7 \%$ and $11 \%$ specimens, respectively.

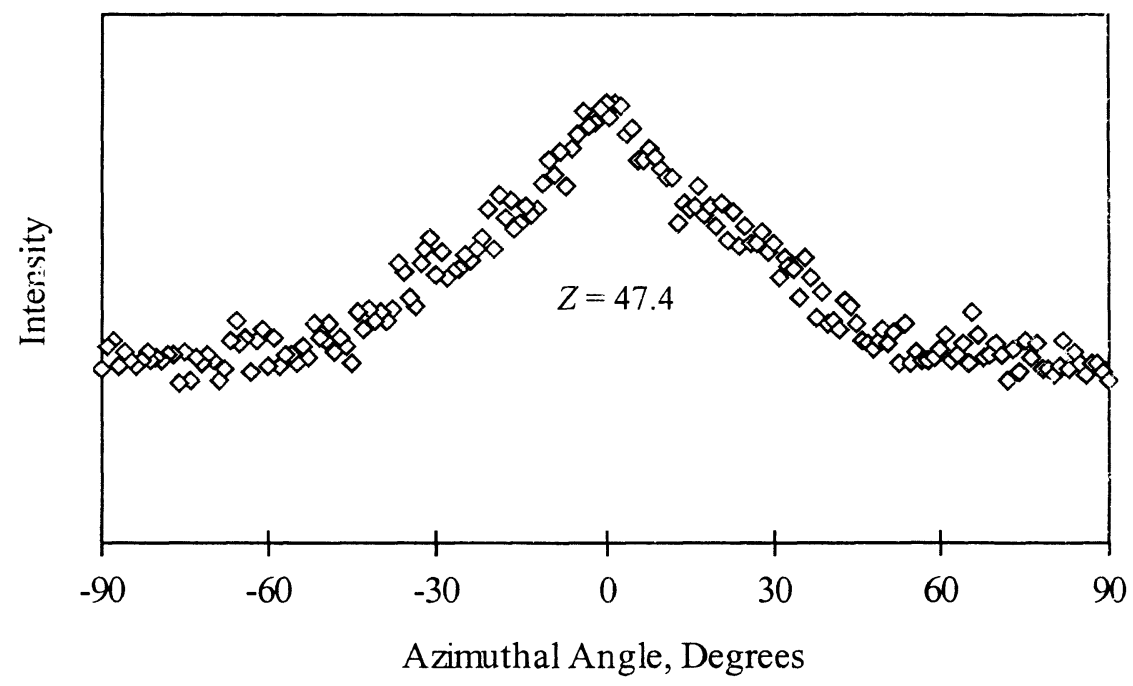

(a) $2.5 \% \mathrm{VGCF}$ 


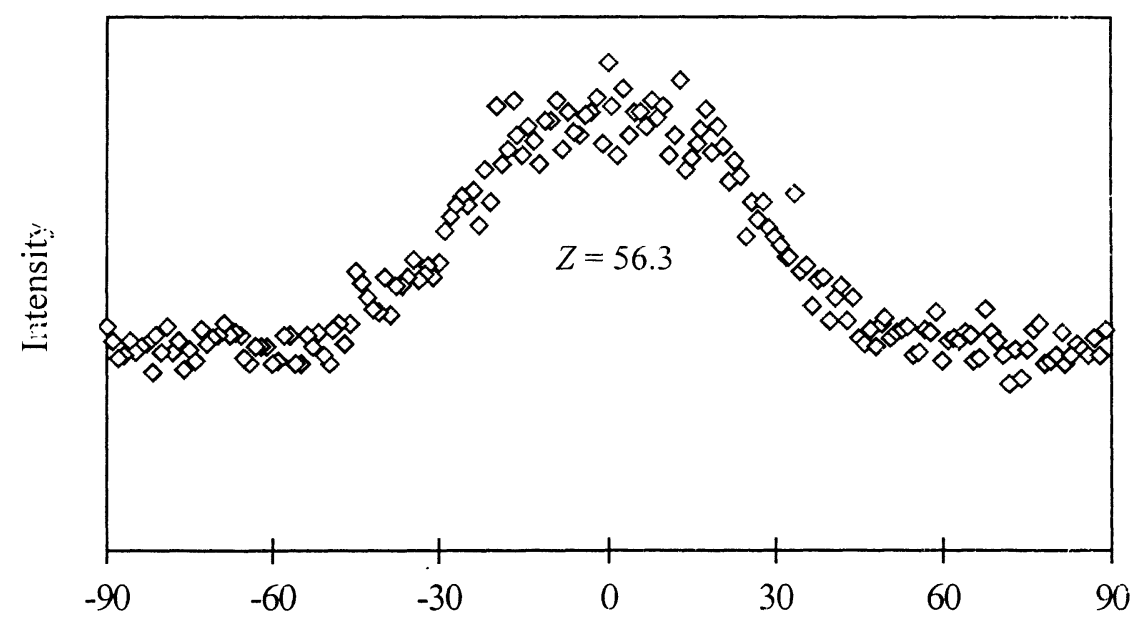

Azimuthal Angle, Degrees

(b) $7 \% \mathrm{VGCF}$

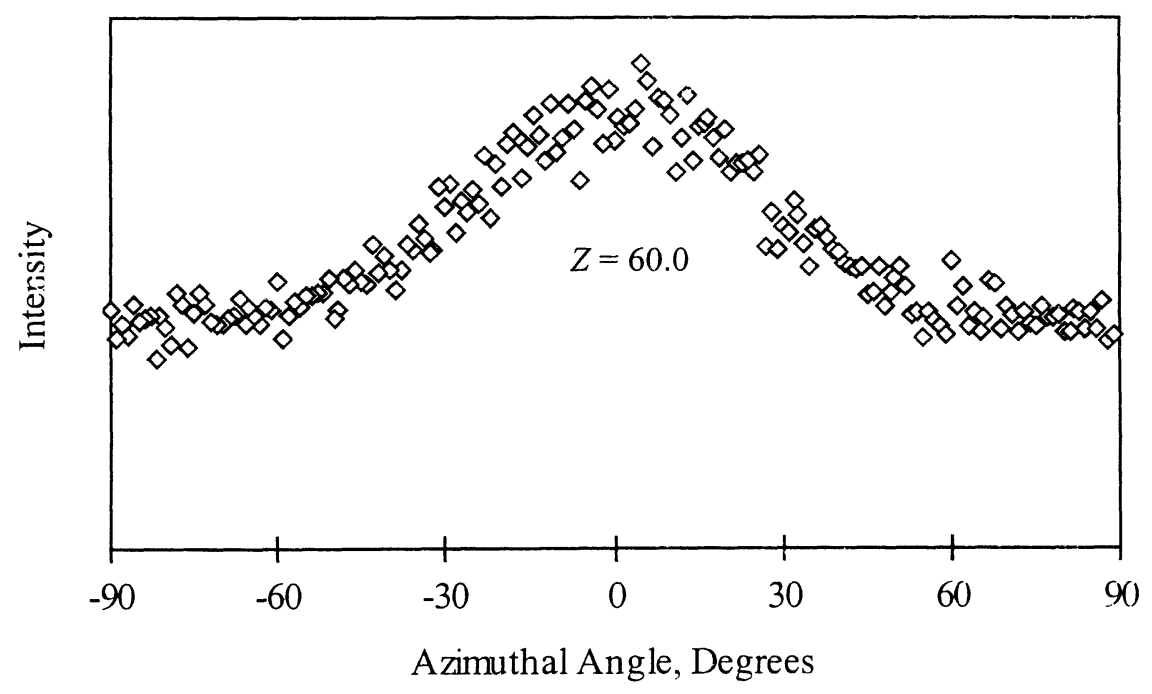

(c) $11 \% \mathrm{VGCF}$

Fig. 4.5. Intensity versus azimuthal angle relative to the preferred axis of composite specimens. 
From the figures it is seen that the degree of fiber alignment tends to decrease as the fiber content increases. As the fiber/polymer mixture is extruded through the converging-annular die several factors affect the state of orientation of fibers suspended in the polymer matrix. Fiber geometry, fiber-fiber and fiber-wall interactions, the suspending fluid rheology, and the flow field all have a direct affect on fiber orientation (Ranganathan and Advani, 1997). As the composite blend is processed through the die, the fibers suspended in a flowing fluid are subjected to local velocity gradients in the fluid. These gradients cause a fluid element to stretch and rotate, thus causing the fiber to rotate in the preferred direction. However, as the fiber volume fraction was increased, the amount of fiber-fiber interaction and fiber-wall interaction also increased. This hinders the fibers' ability to orient themselves along the preferred direction in the flow field.

The amount of fiber alignment along the extrusion direction can be further improved by increasing the residence time of the composite flow through the die channel. The strain field is better able to align the fiber with a larger residence time. As a polymer melt flows through the die channel, the flow strain that is developed near the surface forces the fibers to align with the flow. The theoretical basis of this approach that governs the flow field of a polymer flow is the power law,

$$
\tau=K(\dot{\gamma})^{n}
$$

where $\tau$ and $\dot{\gamma}$ represent the shear stress and shear rate, respectively, with the adjustable parameters being the consistency $K$ and the flow index $\mathrm{n}$. The value of the exponent $n$ is typically of the order 0.3. Such a flow has steep gradients in the region near the walls; 
the extent of this region depends on the power law exponent. Consider the velocity profile for the flow in an annular tube shown in Fig. 4.6.

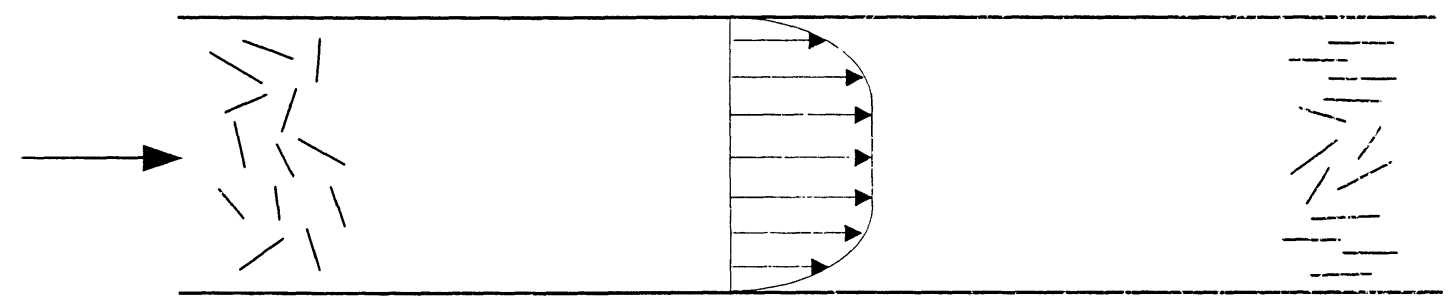

Fig. 4.6. Velocity profile for tube flow and the effect that shear flow has on fiber orientation.

The flow has a much lower velocity near the walls when compared to the center of the tube. This velocity gradient produces a zero shear rate at the center of the flow channel and the highest shear rate occurs along the walls. The reinforcing fibers suspended in the polymer matrix experience the effects of these velocity differentials, thus causing the ends of the fibers nearest to the center of the flow channei to move faster than the ends closer to the wall (Fig. 4.7).

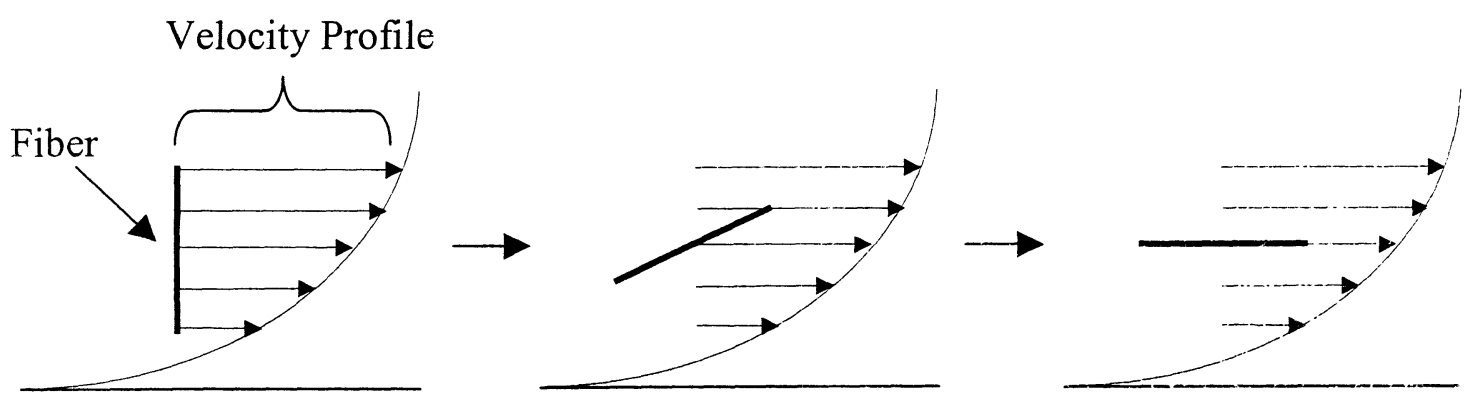

Fig. 4.7. Flow induced alignment of a fiber. 
This will result in progressive alignment of the fiber to bring it in alignment with the flow direction. This effect can also be obtained if extremities such as fins are extended into the flow so that the fiber/polymer mix is forced to flow past the extended surface, resulting in fiber alignment.

The alignment effect is obviously higher if the fiber is longer. When the fiber is longer than the diameter of the flow channel, the fibers must be aligned in order to enter the flow channel. However, for very short fibers, such as the VGCF, a practical value for the channel diameter must be an order of magnitude higher than the fiber length $(0.1$ $\mathrm{mm})$. Experiments have been carried out to determine the extent to which the flow field in a die can orient the fibers. In these experiments, a long thin annular die is used to extrude the fiber/polymer mixture such that a significant fraction of the polymer will experience the high strain field, and fiber alignment is then determined.

To demonstrate this effect, an extended annular nozzle (length $=5.25 \mathrm{~cm}$ ) was attached to the extruder die as shown in Fig. 4.8.

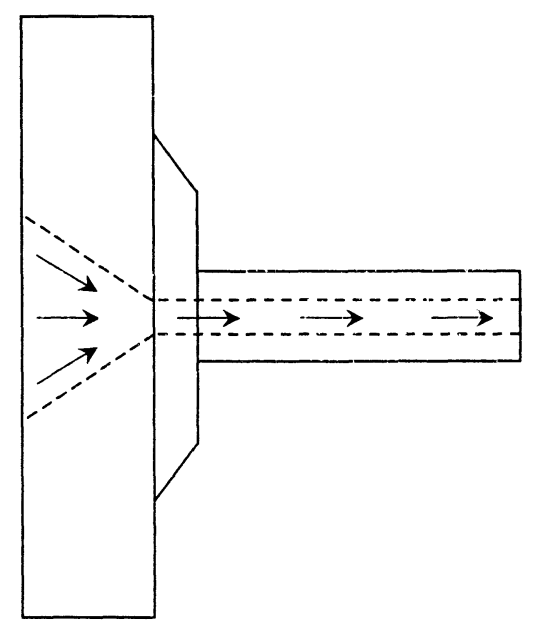

Fig. 4.8. Side view of extruder die with an extended nozzle. 
Composite mixtures were extruded through this die configuration under the same conditions as those processed by the die with no nozzle. This results in a composite product with a higher degree of fiber alignment along the extrusion direction. This can be seen in Fig. 4.9, which gives the diffraction profile for a $2.5 \%$ volume VGCF specimen.

It can be seen from Fig. 4.9 that the fibers are oriented within \pm 15.0 degrees along the preferred direction when the residence time is increased by extruding through the die with a $5.25 \mathrm{~cm}$ long nozzle. This is a $38 \%$ increase in the alignment when compared with the specimens extruded without the nozzle, which aligned the fibers \pm 23.7 degrees along the preferred axis.

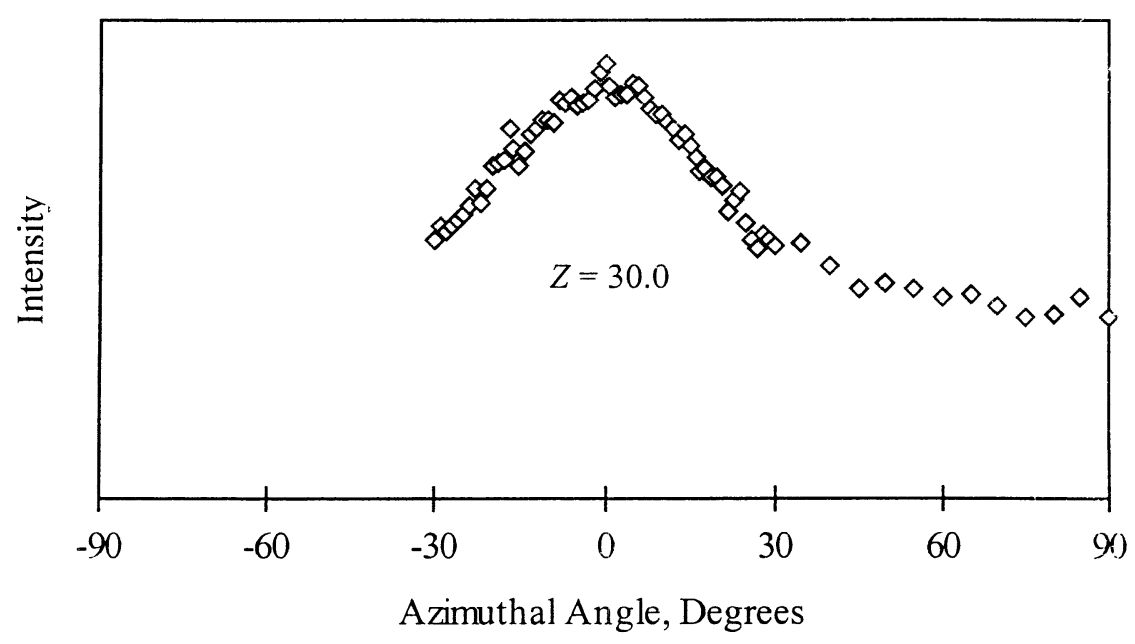

Fig. 4.9. Diffraction profile of a $2.5 \%$ volume VGCF reinforced specimen extruded through an extended nozzle. 
Up to this point only the x-ray diffraction results for the composite strands have been presented. However, the deformation of the composite strands when consolidated by the molding procedure to produce a sheet (as described in Section 3.2) can cause a certain degree of fiber misalignment, which can adversely affect the performance of the composite. The degree of fiber alignment may diminish even more if the sheets were consolidated into a three-dimensional composite cube. This was verified by cutting the molded sheets into $2.5 \mathrm{~cm}$ by $3.8 \mathrm{~cm}$ rectangular pieces. These individual rectangular composite pieces were then stacked into a $2.5 \mathrm{~cm}$ by $3.8 \mathrm{~cm}$ mold (typically $5-7$ pieces) and molded again using the same procedure performed on the sheets, thus producing an anisotropic composite cube. To evaluate how much fiber misalignment the molding processes caused, an X-ray diffraction analysis was performed. Table 4.1 shows the degree of fiber orientation for strands, sheets, and cubes produced by the extrusion and molding processes. From these results it can be seen that each molding procedure reduces the amount of fiber alignment along the preferred direction.

Table 4.1. Fiber orientation along the preferred axis of the composite materials

\begin{tabular}{ccccccc}
\hline & \multicolumn{5}{c}{ Fiber Fraction } & \\
\cline { 2 - 6 } & $2.5 \%$ & $7 \%$ & $9 \%$ & $11 \%$ & $17 \%$ & $23 \%$ \\
\hline Strand & $\pm 22.6^{\circ}$ & $\pm 26.3^{\circ}$ & $\pm 28.3^{\circ}$ & $\pm 30.0^{\circ}$ & $\pm 30.6^{\circ}$ & $\pm 34.7^{\circ}$ \\
Sheet & $\pm 26.5^{\circ}$ & $\pm 29.0^{\circ}$ & - & - & - & - \\
Cube & - & - & $\pm 36.5^{\circ}$ & - & $\pm 38.7^{\circ}$ & $\pm 50.1^{\circ}$ \\
\hline
\end{tabular}




\section{CHAPTER 5}

\section{STRENGTH PREDICTION OF PARTIALLY ALIGNED SHORT FIBER REINFORCED COMPOSITES}

\subsection{Introduction}

In processing short fiber composites, fiber orientation depends on factors such as fiber geometry, fiber-fiber and fiber-wall interactions, the suspending fluid rheology, and the flow field. These factors make it extremely difficult to produce a composite with all fibers perfectly aligned along a specific direction, especially in real-life automated mass production methods, such as injection molding or extrusion. Virtually all manufacturing processes will produce composites that contain fibers partially aligned along the flow direction. This is particularly true for very small fibers such as VGCF, which are also irregular in shape and size. This was shown in Chapter 4 where a composite sample containing only $2.5 \%$ volume fiber could only be aligned $\pm 22.6^{\circ}$ along the preferred axis of the composite strand.

Strength prediction methods for both aligned and randomly oriented short fiber reinforced composites are well established. However, such theoretical models do not accurately predict the strength of composites with its fiber reinforcement partially aligned along a preferred axis. This can be seen in Figs. 5.1 and 5.2 by comparing the strength results of our partially aligned composites along with values from injection molded samples reported by Van Hattum et al. (1999) and Tibbetts and McHugh (1999). The tensile strength was predicted using the theoretical model introduced by Baxter (1992) in 
which the fiber strength was taken to be $2.7 \mathrm{GPa}$ (Glasgow, 2000) and the average fiber length was set equal to 10.42 microns. The modulus was predicted by Cox's model (Cox, 1952) where the modulus of the fiber was taken as $240 \mathrm{GPa}$ (Tibbetts, 2000).

Both the tensile strength and modulus for the samples reinforced with partially aligned fibers increased with fiber volume fraction. The tensile strength increases as the fibers become more aligned by extruding through the $5.25 \mathrm{~cm}$ nozzle attachment. With $11 \%$ fiber volume content there was a $20 \%$ increase in strength when compared to the specimens extruded without the nozzle attachment, and more than double the strength of the polypropylene. As the fiber content is increased, the strength of the samples diverges from the 1-D theoretical model. This occurs because the degree of fiber alignment decreases as fiber loading increases (Table 4.1). This may also be due to an increase in stress concentrations and void content (Gibson, 1994). However, the strength of the aligned fiber composites was still greater than that predicted by the two and threedimensional randomly oriented models, as well as the experimental results of Van Hattum et al. (1999) and Tibbetts and McHugh (1999). 


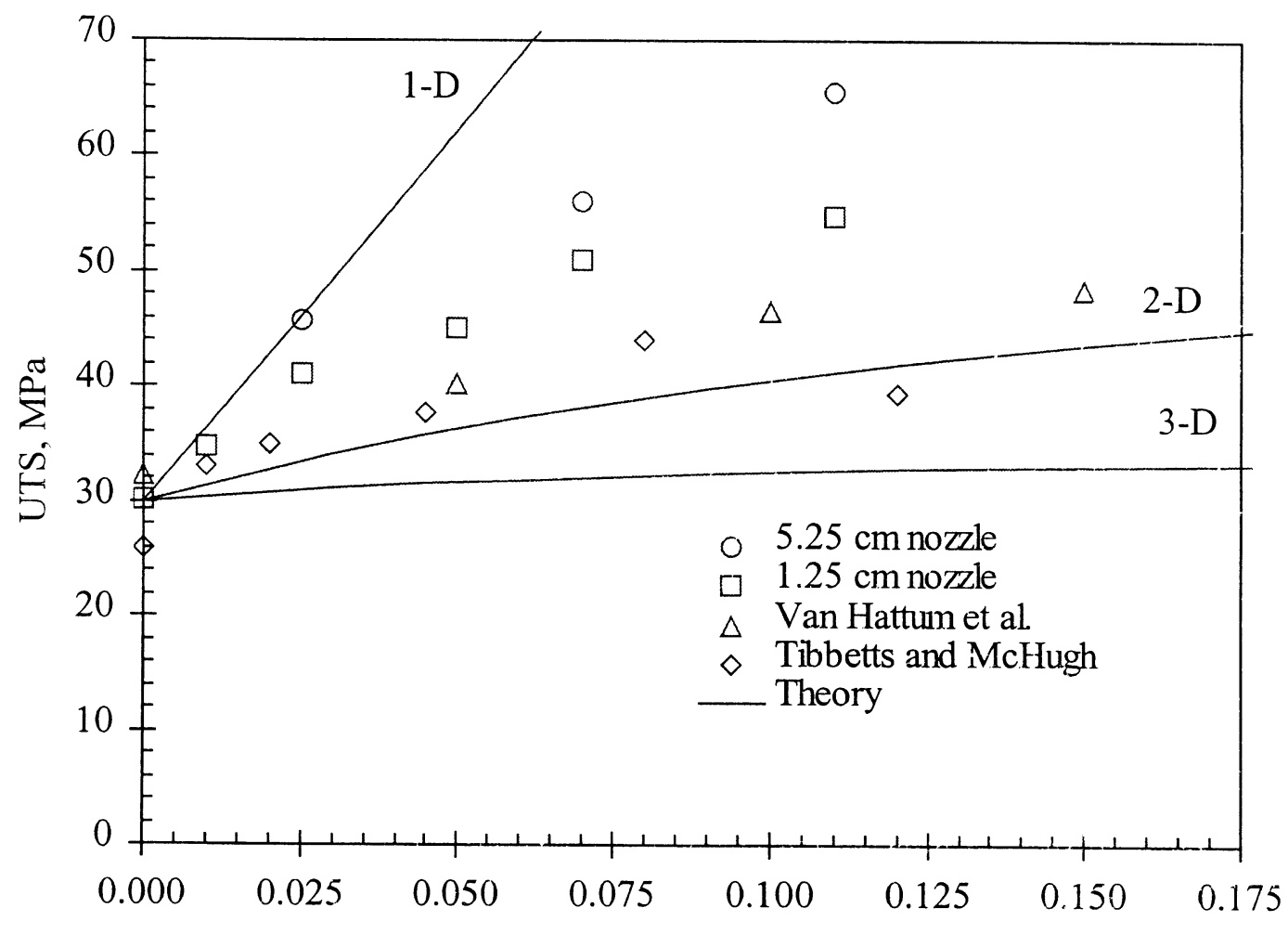

Fiber Volume Fraction

Fig. 5.1. Ultimate tensile strength of polypropylene composites reinforced with partially aligned and randomly oriented VGCF at various fiber volume fractions.

From Fig. 5.2 it is seen that there were generally no large differences between the stiffness of the aligned samples when compared with the results of randomly oriented fiber composites from the other studies and the samples extruded through the nozzle attachment. The stiffness increased with fiber volume fraction; the addition of $11 \%$ by volume VGCF approximately tripled the tensile modulus of pure polypropylene. However, at higher fiber loading the modulus is closer to the theoretical value for 3-D random fiber orientation. Such behavior was also reported by Tibbetts and McHugh 
(1999). It was shown in earlier in Section 3.6, that there is inadequate wetting of the fibers as well as an increase in void concentration at higher fiber loading.

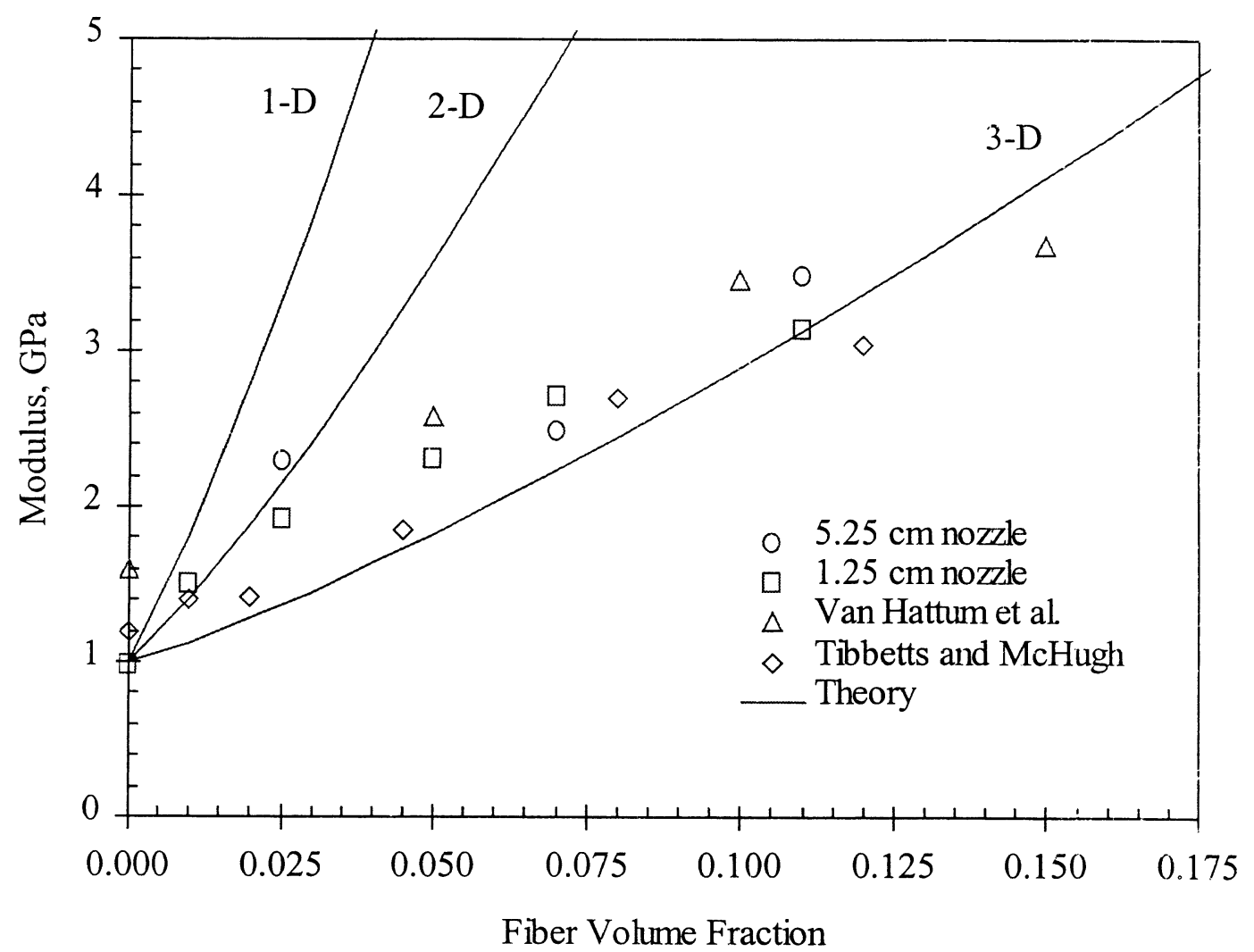

Fig. 5.2. Tensile modulus of polypropylene composites reinforced with partially aligned and randomly oriented at various fiber volume fractions.

Very few methods exist to accurately determine the degree of fiber alignment in a composite material. Therefore, it is generally difficult to predict the strength of a composite with partially aligned short fibers. By applying x-ray diffraction technology it is now possible to accurately determine the fiber distribution in such materials, thus 
making it possible to develop a strength prediction model for partially aligned fiber composites. The work presented in this chapter will introduce such a method.

\subsection{Strength Prediction of Randomly Oriented Fibers}

The approach adopted to model the tensile strength of composites with partially aligned short fibers is based on a method developed by Baxter (1992), which combines micromechanics equations to predict the strength of composites reinforced with randornly oriented discontinuous fibers. Baxter's model makes use of the assumptions made by Lees (1968) that the angular dependence of the applied stress for a randomly oriented fiber composite under uniaxial off-axis loading could be described using the Maximum Stress Criterion where there are three failure mechanisms, each operating over a range of angles. The three failure modes are defined as: fiber failure in tension (Eq. 5.1), shear failure at the fiber/matrix interface (Eq. 5.2), and matrix or interface failure (Eq . 5.3):

$$
\begin{array}{ll}
\text { for small values of } \theta, & \sigma_{1}=\sigma \cos ^{2} \theta \\
\text { for intermediate angles, } & \tau_{12}=\sigma \sin \theta \cos \theta \\
\text { for large angles, } & \sigma_{2}=\sigma \sin ^{2} \theta
\end{array}
$$

These three equations exhibit the biaxial stress-state along the principle material axes with 1 being in the fiber direction and 2 transverse to the fiber. By substituting Eqs. (5.1), (5.2), and (5.3) into the Tsai-Hill Criterion for failure (Azzi and Tsai, 1965; Tsai, 1968) given by,

$$
\left(\frac{\sigma_{1}}{\sigma_{L}}\right)^{2}+\left(\frac{\sigma_{2}}{\sigma_{T}}\right)^{2}-\frac{\sigma_{1} \sigma_{2}}{\sigma_{L}^{2}}+\left(\frac{\tau_{12}}{\tau_{L T}}\right)^{2}=1
$$


We obtain the following expression to predict the strength of a composite $(\sigma)$ as a function of the angle $(\theta)$ between the fiber direction and the applied stress.

$$
\sigma(\theta)=\left[\frac{\cos ^{4} \theta}{\sigma_{L}^{2}}+\left(\frac{1}{\tau_{L T}^{2}}+\frac{1}{\sigma_{L}^{2}}\right) \sin ^{2} \theta \cos ^{2} \theta+\frac{\sin ^{4} \theta}{\sigma_{T}^{2}}\right]^{-1 / 2}
$$

In this expression, $\sigma_{L}, \sigma_{T}$ and $\tau_{L T}$ represent the longitudinal tensile strength, the transverse tensile strength, and the shear strength of the composite material, respectively.

For a continuous fiber reinforced composite, the longitudinal tensile strength $\sigma_{L}$ (when $\theta=0$ degrees) obeys the rule of mixtures,

$$
\sigma_{L}=V_{f} \sigma_{f}+\left(1-V_{f}\right) \sigma_{m}
$$

However, the strengthening of short fibers is less than that from long fibers. This occurs because the ends of the finite-length fibers are stressed less than the maximum iiber stress (Kelly and Tyson, 1965). Interfacial shear stresses along the length of the fibers are caused by the applied stress, and a critical fiber length $\left(l_{c}\right)$ is required for the fibers to reach a maximum stress. If the fiber stress varies linearly with the distance from the fiber end, then Eq. (5.6a) becomes,

$$
\begin{array}{ll}
\sigma_{L}=V_{f} \sigma_{f}\left(1-\frac{l_{c}}{2 l}\right)+\left(1-V_{f}\right) \sigma_{m} & \text { for } l \geq l_{c} \\
\sigma_{L}=V_{f} \sigma_{f} \frac{l}{2 l_{c}}+\left(1-V_{f}\right) \sigma_{m} & \text { for } l \leq l_{c}
\end{array}
$$

where $V_{f}=$ the fiber volume fraction; $l=$ the average fiber length; $\sigma_{f}=$ the fiber strength; $\sigma_{m}=$ matrix strength; and $l_{c}=$ the critical fiber length which is given by, 


$$
\frac{l_{c}}{d}=\frac{\sigma_{f}}{2 \tau_{y}}
$$

where $d$ is the diameter of the fiber and $\tau_{y}$ is equal to the matrix yield stress in shear.

For the transverse tensile strength $\sigma_{T}$ (when $\theta=90$ degrees) there is little or no load sharing between the fiber and matrix, and $\sigma_{T}$ is strongly influenced by the interfacial bond between the two. The fibers can cause stress concentrations in the matrix adjacent to the fibers and thus result in composite failure at stress levels lower than that of unreinforced matrix material. Therefore, there are upper and lower limits for the transverse strength representing two types of interfacial bond behaviors: (i) interfacial bond is weaker than the matrix and (ii) interfacial bond is stronger than the matrix.

When the interfacial bond is weaker than the matrix and the fibers have poor transverse strength, failure occurs by interfacial separation and fiber splitting, respectively. These failure modes have been modeled by Prewo and Kreider (1972) who considered the fibers as cylindrical holes, thus reducing the cross-sectional area of the composite and provides a lower limit for transverse strength,

$$
\sigma_{T}(\min )=\sigma_{m}\left[1-2\left(\frac{V_{f}}{\pi}\right)^{1 / 2}\right]
$$

When the interfacial bond is stronger than the matrix and the fibers do not split, failure occurs in the matrix. Therefore, the upper limit for transverse strength can be taken as,

$$
\sigma_{T}(\max )=\sigma_{m}
$$

By knowing the composite strength as a function of the angle of the applied load, Baxter (1992) used an averaging technique introduced by Lees (1968) and later used by 
Chen (1971) to predict the strength of a randomly oriented fiber composites. This is done by averaging Eq. (5.5) in the following expression,

$$
\sigma_{c}=\frac{2}{\pi} \int_{0}^{\pi / 2} \sigma(\theta) d \theta
$$

which gives the strength of a composite material $\left(\sigma_{c}\right)$ reinforced with randomly oriented fibers.

\subsection{Strength Prediction of Partially Aligned Fibers}

To determine the strength of the composite with partially aligned fibers one must modify Eq. (5.10) to include the degree of fiber alignment. If the fiber alignment information can be determined experimentally, it is possible to derive a strength prediction model for partially aligned short fiber composites. In this study the fiber alignment is determined by utilizing a x-ray diffraction technique. The fiber alignment information is obtained as a Gaussian distribution factor calculated from the data collected from the x-ray diffraction experiments as described earlier in Section 4.3. It should be recalled that the results of the x-ray diffraction analysis provide the fitting parameters as well as the peak area and full-width at half-maximum $(Z)$ used for graphitic misalignment in the carbon fibers. Such results are shown below in Table 5.1. 
Table 5.1. Fiber orientation $(\mathrm{Z} / 2)$ along the preferred axis of the composites samples at various fiber volume percentages.

\begin{tabular}{ccccccc}
\hline & \multicolumn{6}{c}{ Fiber Volume Percentage } \\
\cline { 2 - 7 } & $2.5 \%$ & $7 \%$ & $9 \%$ & $11 \%$ & $17 \%$ & $23 \%$ \\
\hline$Z / 2$ (Degrees) & \pm 23.7 & \pm 26.3 & \pm 27.5 & \pm 30.0 & \pm 30.7 & \pm 35.8 \\
\hline
\end{tabular}

As previously described, the $Z$ values given in Table 5.1 represent the fiber distribution along the preferred axis of the composites samples at various fiber volume percentages. They are given as the full-width at half-maximum intensity peak of the diffraction profile defined by the Gaussian distribution equation,

$$
F(\theta)=\frac{A}{s \sqrt{2 \pi}} e^{-\frac{1}{2}\left(\frac{\theta}{s}\right)^{2}}
$$

where $\theta$ is the azimuthal angle of the misaligned fibers with respect to the preferred axis, and $\mathrm{s}$ is the standard deviation. The standard deviation directly corresponds with $Z$, and the relationship between the two can be solved by referring to Fig. 5.3 and evaluating the coefficient $A$ and the standard deviation $s$ as follows: 


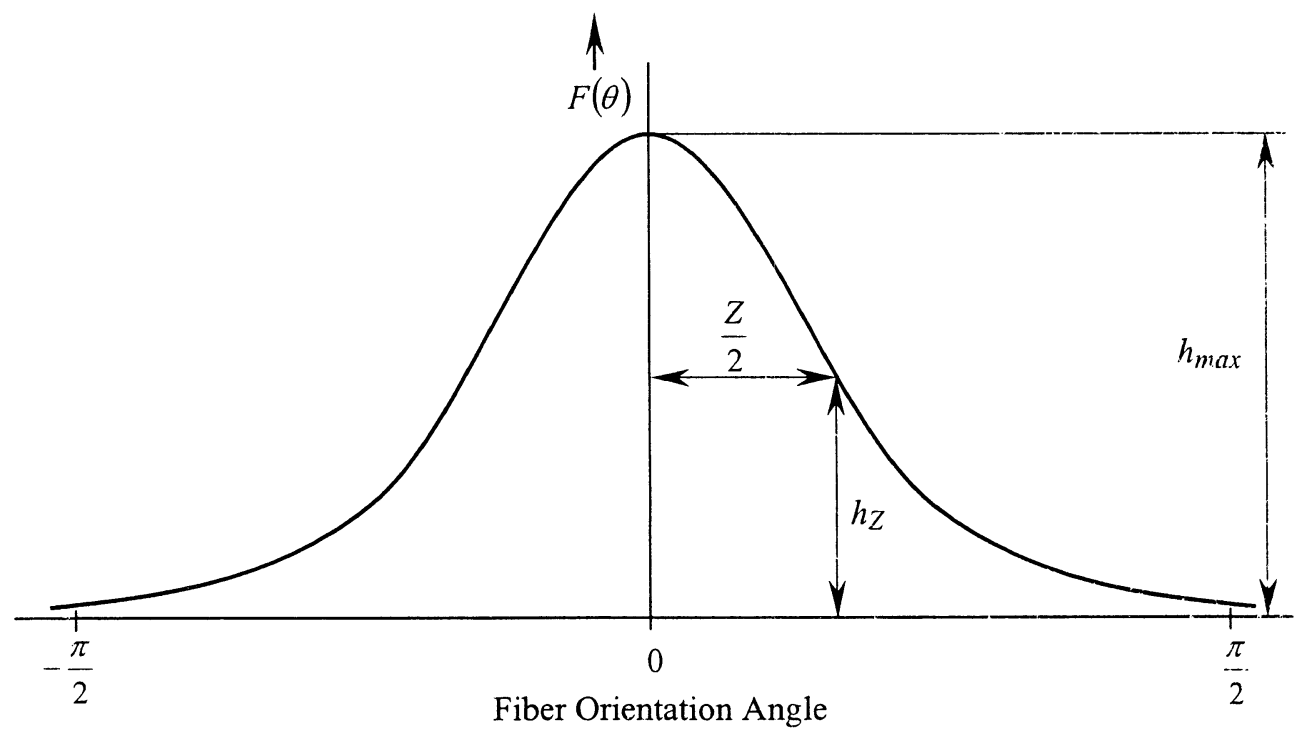

Fig. 5.3. Gaussian distribution function $F(\theta)$ of the fiber orientation with respect to axial direction. The axial direction is $\theta=0 . Z$ is the full width of the diffraction intensity measured at one half the maximum intensity.

Since $Z$ is given as the full-width at half the maximum peak of the distribution curve, the following relationship can be introduced,

$$
h_{Z}=\frac{1}{2} h_{\max }
$$

where

$$
\begin{gathered}
h_{\max }=F(0)=\frac{A}{s \sqrt{2 \pi}} \\
h_{Z}=F\left(\frac{Z}{2}\right)=\frac{A}{s \sqrt{2 \pi}} e^{-\frac{1}{2}\left(\frac{Z}{2 s}\right)^{2}}
\end{gathered}
$$

Substituting Eqs. (5.13) and (5.14) into Eq. (5.12) gives 


$$
\frac{A}{s \sqrt{2 \pi}}=\frac{A}{2 s \sqrt{2 \pi}} e^{-\frac{1}{2}\left(\frac{Z}{2 s}\right)^{2}}
$$

Solving Eq. (5.15) for the standard deviation $s$, yields

$$
\mathrm{s}=\frac{\mathrm{Z}}{2.35}
$$

The coefficient $A$ is included in Eq. (5.11) because we must have

$$
\int_{-\pi / 2}^{\pi / 2} F(\theta) d \theta=1
$$

By substituting Eq. (5.11) into Eq. (5.17) we can solve for $A$ by letting

$$
u=\frac{\theta}{s} \quad d u=\frac{d \theta}{s} \quad d \theta=s d \theta
$$

therefore when $\theta= \pm \frac{\pi}{2}, u= \pm \frac{\pi}{2 s}$

$$
\frac{A}{s \sqrt{2 \pi}} \int_{-\pi / 2}^{\pi / 2} e^{-\frac{1}{2}\left(\frac{\theta}{s}\right)^{2}} d \theta=\frac{A}{\sqrt{2 \pi}} \int_{-\pi / 2 s}^{\pi / 2 s} e^{-\frac{1}{2} u^{2}} d u \equiv 1
$$

which give

$$
A=\frac{\sqrt{2 \pi}}{\int_{-\pi / 2 s}^{\pi / 2 s} e^{-\frac{1}{2} u^{2}} d u}
$$

For the experimental data in this work, the value of $A$ was found to be very close to unity. By knowing the relationship between the standard deviation and the $Z$ value (Eq. (5.16)), and setting $A$ equal to 1 , the Gaussian distribution equation can be written as 


$$
F(\theta)=\frac{2.35}{Z \sqrt{2 \pi}} e^{-\frac{1}{2}\left(\frac{2.35 \theta}{Z}\right)^{2}}
$$

and incorporated into Eq. (5.10) to serve as a weighting factor that accounts for fiber orientation. The strength of the composite $\left(\sigma_{c}\right)$ with partially aligned short fibers is given by

$$
\sigma_{c}=2 \int_{0}^{\pi / 2} F(\theta) \sigma(\theta) d \theta
$$

with $\sigma(\theta)$ as written in Eq. (5.5).

\subsection{Theoretical Results}

To evaluate the composite strength according to Eq. (5.21) it is essential to know the properties $\left(\sigma_{f}, l / d, l_{c}\right)$ and the content $\left(V_{f}\right)$ of the fibers as well as the strength of the matrix $\left(\sigma_{m}\right)$ used for the composite samples. The strength $\left(\sigma_{m}\right)$ of the polypropylene matrix used in this study was found to be $30 \mathrm{MPa}$ by testing samples that were processed in the same manner as the composite material. The VGCF reinforcement had diameters (d) of approximately 0.2 micrometers and an ultimate strength $\left(\sigma_{f}\right)$ of $2.7 \mathrm{GPa}$ (Glasgow, 2000). The critical fiber length/fiber diameter $\left(l_{c} / d\right)$ ratio is approximately equal to 100 , therefore $l_{c}$ is 20 micrometers (Tibbetts, 2000). The average fiber length $(l)$ was taken to be 10.42 microns on the basis described earlier in Section 3.4.

Since the average fiber length of 10.42 microns is less than the critical fiber length $\left(l_{c}=20\right.$ microns), Eq. (5.6c) is appropriate for the longitudinal composite strength. 
Also, previous work has shown that interfacial bonding between polypropylene and VGCF is generally quite strong (Tibbetts, 2000; Glasgow, 2000). Therefore, failure in the transverse direction will occur in the adjacent polymer matrix so the transverse strength $\left(\sigma_{T}\right)$ will equal $30 \mathrm{MPa}$, the strength of the polypropylene (Eq. 5.9). The tensile strength as a function of the angle between the aligned fiber axis and the applied stress (Eq. 5.5) can now be solved with $\tau_{L T}$ taken as $1 / \sqrt{3}$ times the matrix tensile strength, which sets $\sigma$ equal to the matrix strength at $0 \%$ fiber volume. Calculations for partially aligned fiber composites can now be made using Eq. (5.16). Since this equation can not be integrated in closed form, it was solved numerically within a relative error of $1 \times 10^{-3}$ using an adaptive recursive Newton-Cotes 8 panel rule.

The effects of fiber length and fiber volume fraction on the composite strength have been calculated using Eq. (5.21) and are illustrated in Figs. 5.4 and 5.5. These figures clearly show that the composite strength increases as the amount of fiber alignment $(Z)$ increases. From Fig. 5.4 it is also seen that the strength increases as the fiber length (aspect ratio, $l / d$ ) increases. As expected, the composite strength is more sensitive to variation in fiber length when the average fiber length is less than the critical fiber length $\left(l<l_{c}\right)$. The composite strength is also improved as the fiber content increases. Figure 5.5 shows the variation of composite strength as a function of the fiber volume fraction and the degree of fiber alignment. 


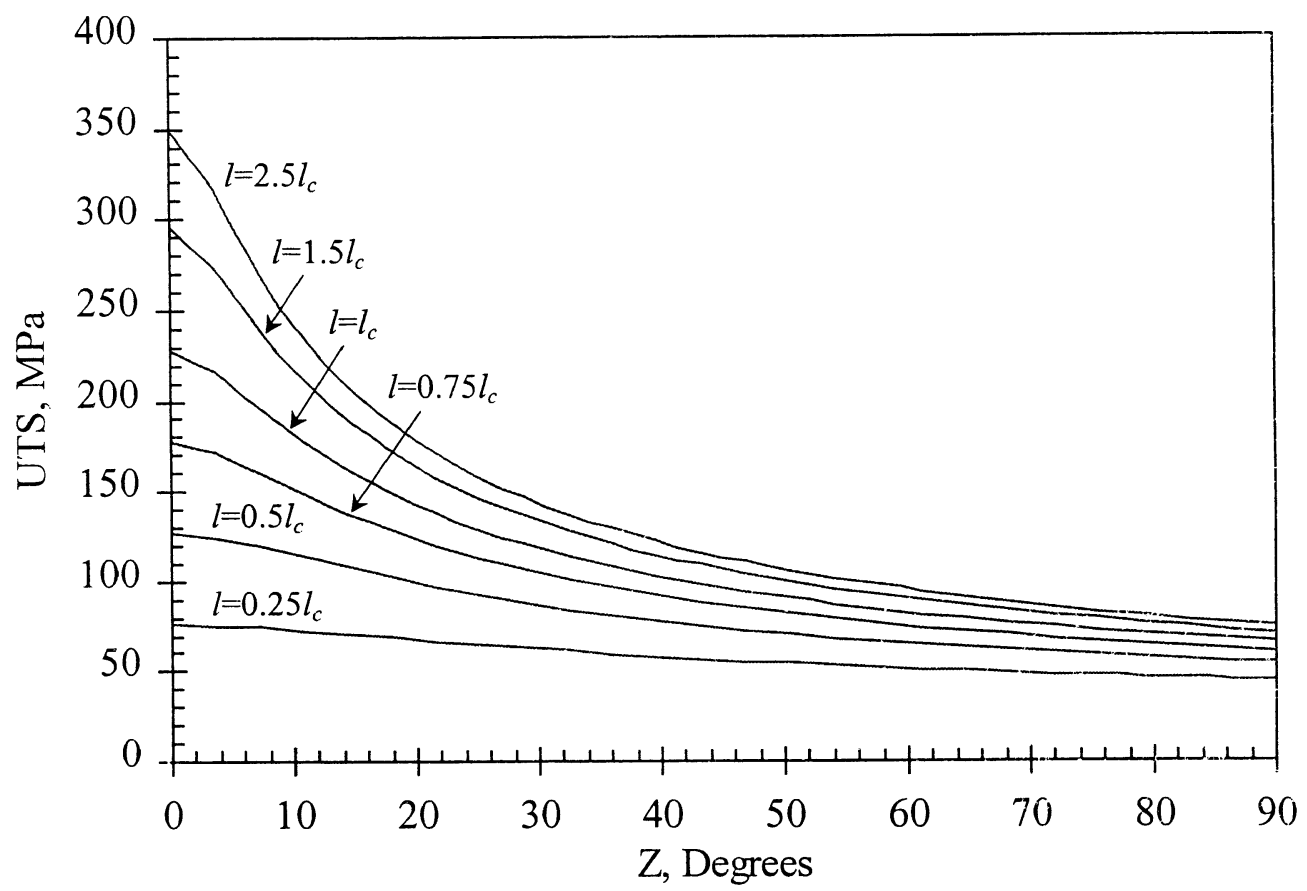

Fig. 5.4. The predicted ultimate tensile strengths of polypropylene reinforced with $15 \%$ volume VGCF as a function of fiber alignment and fiber length.

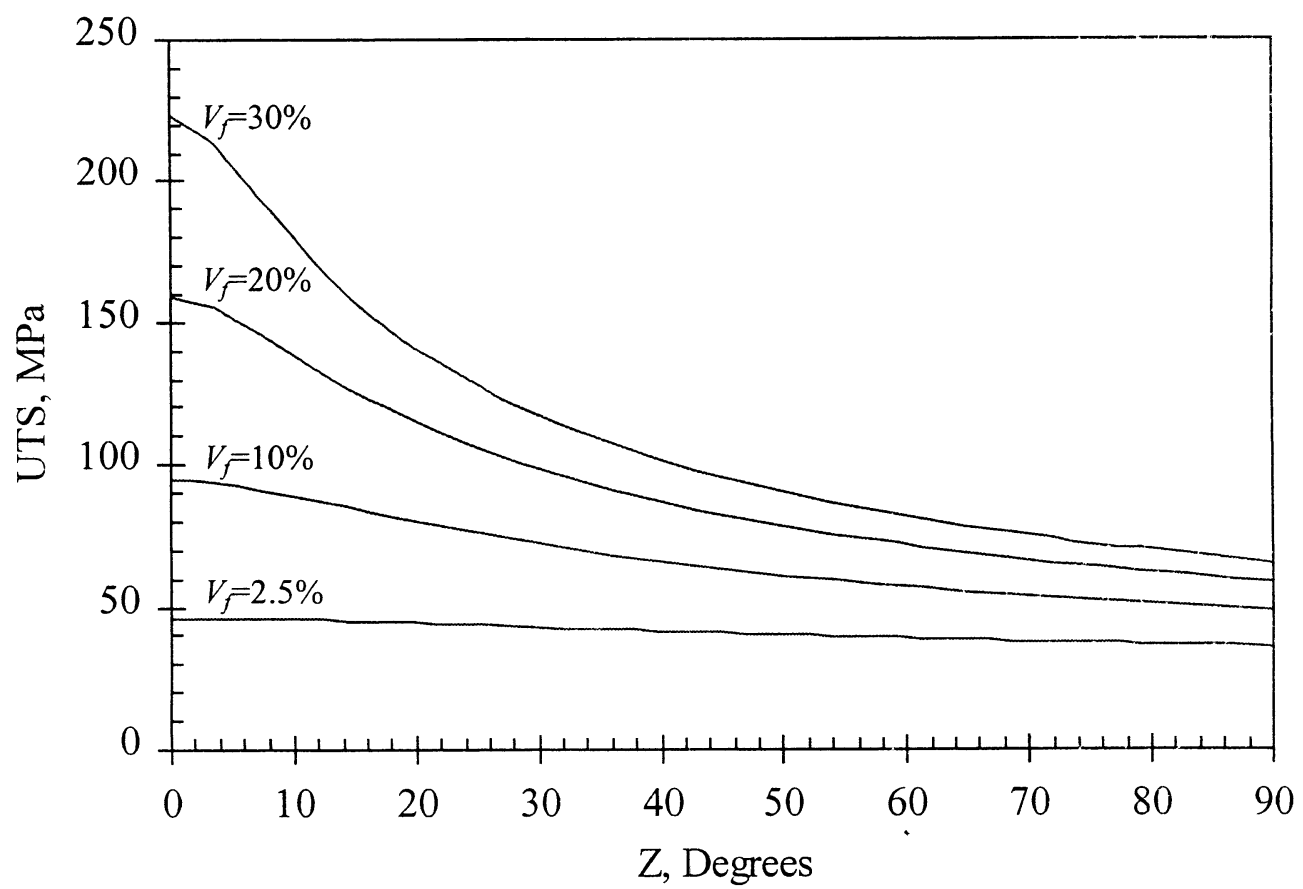

Fig. 5.5. The predicted UTS of a partially aligned VGCF reinforced polypropylene as a function of fiber volume fractions $(l=10 \mu \mathrm{m})$ and degree of alignment. 


\subsection{Comparison of Experiment and Theory}

Figure 5.6 shows the results of the analytical calculations compared with the experimental measurements. Theoretical curves were determined with an average fiber length $(l)$ equal to 5, 10 and 15 micrometers, and are given to show the sensitivity of the composite strength to the average fiber length. These results show that the experimental data corresponds very well with the analytical results calculated with $l=10$ micrometers, which was the approximate average length found from the TEM analysis (as described in Section 3.4).



Fig. 5.6. Ultimate tensile strength of partially aligned VGCF reinforced polypropylene with various fiber volume fractions and various average fiber lengths. The experimental values are compared with the theoretical curve given by Eq. (5.21). 
It is obvious that the tensile strength increases with fiber volume fraction and is much more sensitive at lower levels of fiber loading. However, as fiber loading increases, the strengthening effect is not as great and can even cause a reciuction in strength. This is evident in the Fig. 5.6 where the samples reinforced with $23 \%$ fiber volume fraction are significantly lower than the predicted value. This is most likely due to incomplete fiber wetting and infiltration because of high fiber loading and high viscosity of the fiber/polymer mixture. 


\section{CHAPTER 6}

\section{TRANSPORT PROPERTIES OF VAPOR GROWN CARBON FIBER COMPOSITES}

\subsection{Introduction}

Up to this point we have focussed primarily on using VGCF for improving the mechanical properties of composite materials. However, the unique characteristics of VGCF also gives them thermal and electrical conductivities which are superior to that of conventional carbon fibers and is to be considered one of the best conductive carbon reinforcements for polymer composites (Yamanashi et al., 1993). VGCF is highly graphitic with a lamellar morphology and physical properties approaching that of single-

crystal graphite (Lake et al., 1992). Due to the purity in which carbon is incorporated into the crystal lattice and its unique morphology, VGCF can be highly graphitized by heat treatment. The heat-treating process improves the graphitic crystallite structure of the fiber, thus increasing the conductive properties of the fiber.

The fibers are composed of concentric basal planes that are parailel to the fiber axis giving an annular geometry (Fig. 2.5), opposed to the radial atomic plane geometry that is characteristic of conventional carbon fibers. Therefore, it can be expected that the conductive properties along the fiber axis can be very different from the transverse direction. For instance, the measured thermal conductivity and electrical resistivity of the larger Pyrograf IM VGCF is approximately $20 \mathrm{~W} / \mathrm{m}-\mathrm{K}$ and $1000 \mu \mathrm{Ohm}-\mathrm{cm}$, respectively, opposed to $1950 \mathrm{~W} / \mathrm{m}-\mathrm{K}$ and $55 \mu \mathrm{Ohm}-\mathrm{cm}$ for the heat-treated fiber (ASI, 1999). This 
difference adds to the variation of conductive properties that would occur if an isotropic fiber was used for composite reinforcement.

Many commercial markets could benefit from the conductive properties of VGCF composites, particularly the electronics, aerospace, and automotive industries. For example, VGCF composites could be used for thermal management applications such as electronic packaging. With the increasing power output levels required for future advanced electronic systems, the current materials used for electronic packaging will need to be improved to prevent failure of the electronic components due to overheating. Also, the high electrical conductivity of VGCF composites makes them an attractive material for electrode plates used in batteries and fuel cells, since they are light, strong, vibration and shock resistant, and electrically conductive. Other proposed applications of VGCF composites are for a wide variety of structural components, heat sinks, rubber compounds, and coatings just to name a few (Lake et al., 1992).

A number of studies have been carried out to evaluate the conductive properties of VGCF reinforced composites (Yamanashi, 1993; Dasch et al., 1993; Ali et al., 1995; Ciminelli et al., 1996; Zhang et al., 1998; Patton et al., 1999). The results of these studies show that the thermal and electrical conductivities of the matrices were increased by the addition of VGCF. However, all experimental data gathered from VGCF composites are with the fibers randomly oriented in the matrix material (certain amounts of fiber orientation may occur during the processing of the composites). As reported in Chapters 4 and 5 , the strength properties of the extruded polypropylene were greatly improved by introducing aligned VGCF, and were consistently higher than the specimens reinforced 
with randomly oriented fibers. The conductive properties of a composite can also be improved by fiber alignment. In the case of discontinuous fibers such as VGCF, the energy is transferred form one fiber to the other either by physical contact or through the matrix material. Therefore, when the fibers are well dispersed and aligned in the matrix, they form a fine network or continuous pathway that reduces contact resistance and increases conductivity. Moreover, the shape and concentration of the fiber particles can also influence the conductivity properties of a composite. Composites becorne thermally and electrically conductive at a critical volume concentration of conductive fillers. The critical concentration can be reduced to lower levels by using conductive fillers that are fibrous in shape and have large aspect ratios, which is characteristic of VGCF that can have fiber aspect ratios $(l / d)$ ranging from 100 to 400 . However, VGC'F is irregular in shape and not straight like traditional short fibers.

In this chapter, the thermal and electrical properties of composites VGCF are investigated. The following work will show that an aligned VGCF reinforced thermoplastic composite can be fabricated with excellent thermal and electrical properties.

\subsection{Experimental Thermal Analysis}

By knowing the density, specific heat, and thermal diffusivity of a material, the thermal conductivity of that material can be determined. This is demonstrated by evaluating the thermal conductivity of the anisotropic composite cube samples fabricated 
as described in Section 4.3 containing various fiber volume fractions of the PR-19-HT fiber.

Thermal diffusivity measurements were made on the composite samples using a Holometrix $\mu$ Flash Thermal Properties Instrument. Disc-shaped test samples (12.5 mm diameter and $2 \mathrm{~mm}$ thick) were machined from the cubes to fit the instrument's sample holder. The instrument uses a neodymium-glass laser to uniformly irradiate one side of the test sample using a single laser beam pulse. The laser pulse provides 10 Joules of energy at wavelengths of 1060 nanometers for 400 microseconds. The temperature rise on the other side (or rear face) is then measured as a function of time using a mercurycadmium-telluride infrared detector cooled by liquid nitrogen. The thermal diffusivity can then be determined by comparing the measured data with the appropriate mathematical model. The simplest method for calculation of the thermal diffusivity was developed by Parker et al. (1961) who assumed that the sample is perfectly insulated from the environment and all the laser energy was absorbed into the sample material facing the laser. Under these assumptions, the thermal diffusivity $(\alpha)$ can be calculated based on the time needed for the rear face to reach $50 \%$ of its maximum temperature,

$$
\alpha=\frac{0.1388 y^{2}}{t_{50}}
$$

where $y$ is the sample thickness and $t_{50}$ is the time needed to reach $50 \%$ of the maximum temperature.

However, the above equation must be modified to account for heat loss and other variables. When there is heat loss from the sample, the temperature rise reaches its peak 
at a lower temperature than without heat loss, and the temperature drops off after the peak. For the current study, the heat losses are accounted for by a method introduced by Cowen (1963). Cowen developed a theoretical relationship between the dimensionless parameter,

$$
P=\frac{\alpha t_{50}}{y^{2}}
$$

and the ratio of two dimensionless temperatures obtained from the temperature rise curve,

$$
\frac{\theta\left(5 t_{50}\right)}{\theta\left(t_{50}\right)}
$$

The value $\theta\left(5 t_{50}\right)$ is the fraction of the peak temperature rise at a time equal to 5 times $t_{50}$, and the dimensionless temperature $\theta\left(t_{50}\right)=0.5$.

When there is no heat loss from the sample, the temperature remains constant after reaching the peak and the ratio given in Eq. (6.3) becomes 2.0, while the dimensionless $P$ parameter in Eq. (6.2) becomes 0.1388 as in the Parker analysis. The method analyzes the temperature following the peak where the sample is cooling off, and uses the dimensionless temperature ratio in Eq. (6.3) to describe the temperature curve when there is heat loss during the test. The method is incorporated into the data acquisition program supplied by Holometrix, and provides an accurate measurement for thermal diffusivity.

The laser flash measurements were repeated several times on the test samples to establish the correct response times. The results from several measurements were averaged to obtain the values of thermal diffusivity for the composite materials in the 
longitudinal $\left(\alpha_{L}\right)$ and transverse $\left(\alpha_{T}\right)$ directions. By knowing the thermal diffusivity value, the effective thermal conductivity can then be calculated using,

$$
k_{L}=\alpha_{L} \rho_{c} C_{c} \quad \text { and } \quad k_{T}=\alpha_{T} \rho_{c} C_{c}
$$

where $C_{c}$ and $\rho_{c}$ is the specific heat and density of the composite materials, respectively.

The density of the composite cubes was experimentally determined according to ASTM D 792 Test Method A guidelines (ASTM, 1993). Test samples were cut from the cubes and varied in size, typically weighing between 1 and 2 grams. A balance with an accuracy of $0.1 \mathrm{mg}$ was used to measure the mass of the samples. A fine wire connected to the balance was attached to the test specimen so it could be submerged in a beaker of distilled water at $23^{\circ} \mathrm{C}$ (samples lighter than water required a sinker attached to the wire for immersion). The apparent mass of the immersed specimen, wire, and sinker was determined. The specimen was then removed from the set-up so the apparent mass of immersed wire and sinker could be measured. The density of the composite $\rho_{c}$ was calculated using the following,

$$
\rho_{c}=997.6 \times \frac{a}{(a+w-b)}
$$

where, $a=$ mass of the specimen; $b=$ mass of the specimen, wire, and sinker (when used) when immersed in water; and $w=$ mass of the wire and sinker (when used) when immersed in water.

The specific heat $C_{c}$ was experimentally determined using a Perkin Elmer, Pyris 1 Differential Scanning Calorimeter (DSC). Specific heat is a fully normalized output of the DSC, that is, the displacement during a scan is equal to the product of the heat 
capacity times the sample weight times the scanning rate plus the no-sample baseline run. Therefore, no-sample baseline runs were made using the same sample pans and conditions used for the actual test samples. This constitutes the classical two-curve specific heat method and provides accurate results, which are calculated by the software that accompanies the DSC. Test specimens were made from the cubes to fit into the $0.635 \mathrm{~cm}$ diameter aluminum sample pan. The specimens were approximately $0.5 \mathrm{~mm}$ thick and weighed between 10 and $25 \mathrm{mg}$ depending on the fiber content. Each specimen was encapsulated in the sample pan used in the baseline run for that particular analysis. The specimens were heated to $70^{\circ} \mathrm{C}$ at a rate of $10^{\circ} \mathrm{C} / \mathrm{min}$ while the sample chamber was purged with nitrogen at $20 \mathrm{cc} / \mathrm{min}$ and cooled by an ice bath.

The experimental results for the density, specific heat, and thermal diffusivity are shown in Table 6.1 along with the resulting thermal conductivity values. As expected, the density increases with fiber content since the fibers are denser than polymer matrix. On the other hand, the specific heat of the fibers is much lower than that of the polypropylene, thus resulting in a lower specific heat for the samples at higher fiber contents. The anisotropy of the thermal properties of VGCF composites is also evident, where the longitudinal diffusivity and the resultant conductivity is seen to be much higher than the transverse diffusivity; although both quantities increased with fiber content. 
Table 6.1. Thermal properties of PR-19-HT Pyrograf III ${ }^{T M}$ fiber reinforced polypropylene at various fiber volume fractions.

\begin{tabular}{lcccc}
\hline & \multicolumn{4}{c}{ Fiber Volume Percent } \\
\cline { 2 - 5 } \multicolumn{1}{c}{ Property } & $0 \%$ & $9 \%$ & $17 \%$ & $23 \%$ \\
\hline Density $\left(\mathrm{kg} / \mathrm{m}^{3}\right)$ & 908 & 1012 & 1074 & 1143 \\
Specific Heat $(\mathrm{J} / \mathrm{kg}-\mathrm{K})$ & 1826 & 1652 & 1519 & 1475 \\
Thermal Diffusivity $\left(\mathrm{cm}^{2} / \mathrm{s}\right)$ & & & & \\
$\quad$ Longitudinal $\left(\alpha_{L}\right)$ & 0.00120 & 0.01102 & 0.02482 & 0.03509 \\
$\quad$ Transverse $\left(\alpha_{T}\right)$ & 0.00121 & 0.00974 & 0.01246 & 0.01348 \\
Thermal Conductivity $(\mathrm{W} / \mathrm{m}-\mathrm{K})$ & & & & \\
$\quad$ Longitudinal $\left(k_{L}\right)$ & 0.199 & 1.841 & 4.050 & 5.916 \\
$\quad$ Transverse $\left(k_{T}\right)$ & 0.201 & 1.628 & 2.033 & 2.273 \\
\hline
\end{tabular}

\subsection{Theoretical Thermal Analysis}

The experimental results shown in Table 6.1 can be verified using known theoretical models. For example, the theoretical values for density and specific heat can be obtained by the rule of mixtures. The density of a composite material $\left(\rho_{c}\right)$ with a fiber volume fraction $V_{f}$ is found by

$$
\rho_{c}=V_{f} \rho_{f}+\left(1-V_{f}\right) \rho_{m}
$$

where $\rho_{f}$ and $\rho_{m}$ represent the density of the fiber and matrix materials, respectively. From the experimental results the density of the polypropylene matrix is known to be 908 $\mathrm{kg} / \mathrm{m}^{3}$ (Table 6.1). However, the fiber density and all other properties of the PR-19-HT Pyrograf III ${ }^{\mathrm{TM}}$ fiber are relatively unknown. As discussed in Chapter 2, the microscopic 
size and irregular nature of the nanometer-size Pyrograf III $^{\mathrm{TM}}$ fiber makes it difficult to perform direct measurements to determine the bulk properties of the fibers. Therefore, the properties of the similar, but larger Pyrograf $\mathrm{I}^{\mathrm{TM}}$ fiber are commonly used to represent those of Pyrograf III ${ }^{T M}$. But it is uncertain if the thermal properties of the Pyrograf III ${ }^{\top M}$ fiber are the same as the properties of Pyrograf $I^{\mathrm{TM}}$ fiber. This can be verified in Fig. 6.1 by assuming the density of the fiber to be $2000 \mathrm{~kg} / \mathrm{m}^{3}$, which is the measured density of the Pyrograf $I^{T M}$ fiber (ASI, 1999).

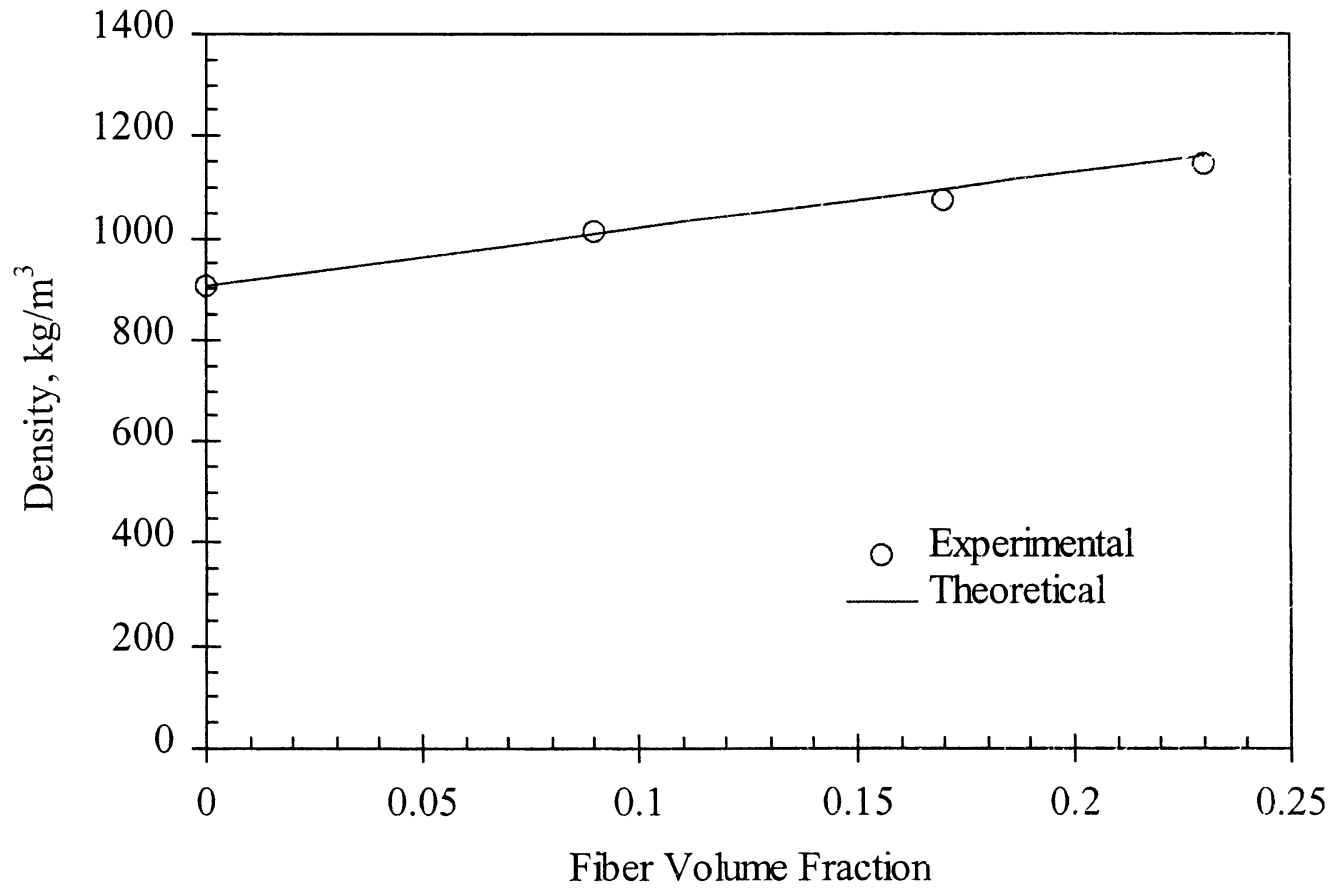

Fig. 6.1. Experimental density values of polypropylene reinforced with various amounts of Pyrograf III ${ }^{\mathrm{TM}}$ (PR-19-HT) VGCF. These values are compared with theoretical results calculated assuming the fiber density equal to that of Pyrograf $\mathrm{I}^{\mathrm{TM}}$ fiber. 
The results in Fig. 6.1 show that the experimental and analytical results correspond extremely well, with none differing by more than $2 \%$. This suggests that the density of the Pyrograf III ${ }^{T M}$ fiber is similar to that of the Pyrograf $I^{T M}$ fiber and a value of 2000 $\mathrm{kg} / \mathrm{m}^{3}$ is a reasonable assumption.

In a similar fashion, the heat capacity can be found by

$$
C_{c}=\frac{1}{\rho_{c}}\left[V_{f} \rho_{f} C_{f}+\left(1-V_{f}\right) \rho_{m} C_{m}\right]
$$

where $C_{f}$ and $C_{m}$ represent the specific heat of the fiber and the matrix material, respectively. As with the density, the heat capacity of the fiber will be set equal to 710 $\mathrm{J} / \mathrm{kg}-\mathrm{K}$, which is the measured value of the Pyrograf I ${ }^{\mathrm{TM}}$ fiber (ASI, 2000). The specific heat of the polypropylene matrix was found to be $1826 \mathrm{~J} / \mathrm{kg}-\mathrm{K}$ (Table 6.1). By comparing the experimental data and the analytical results found from Eq. (6.7) in Fig. 6.2 , it can be seen that the results are very similar. This suggests that the specific heat of Pyrograf III ${ }^{\mathrm{MM}}$ is comparable to the Pyrograf $\mathrm{I}^{\mathrm{TM}}$ fiber. 


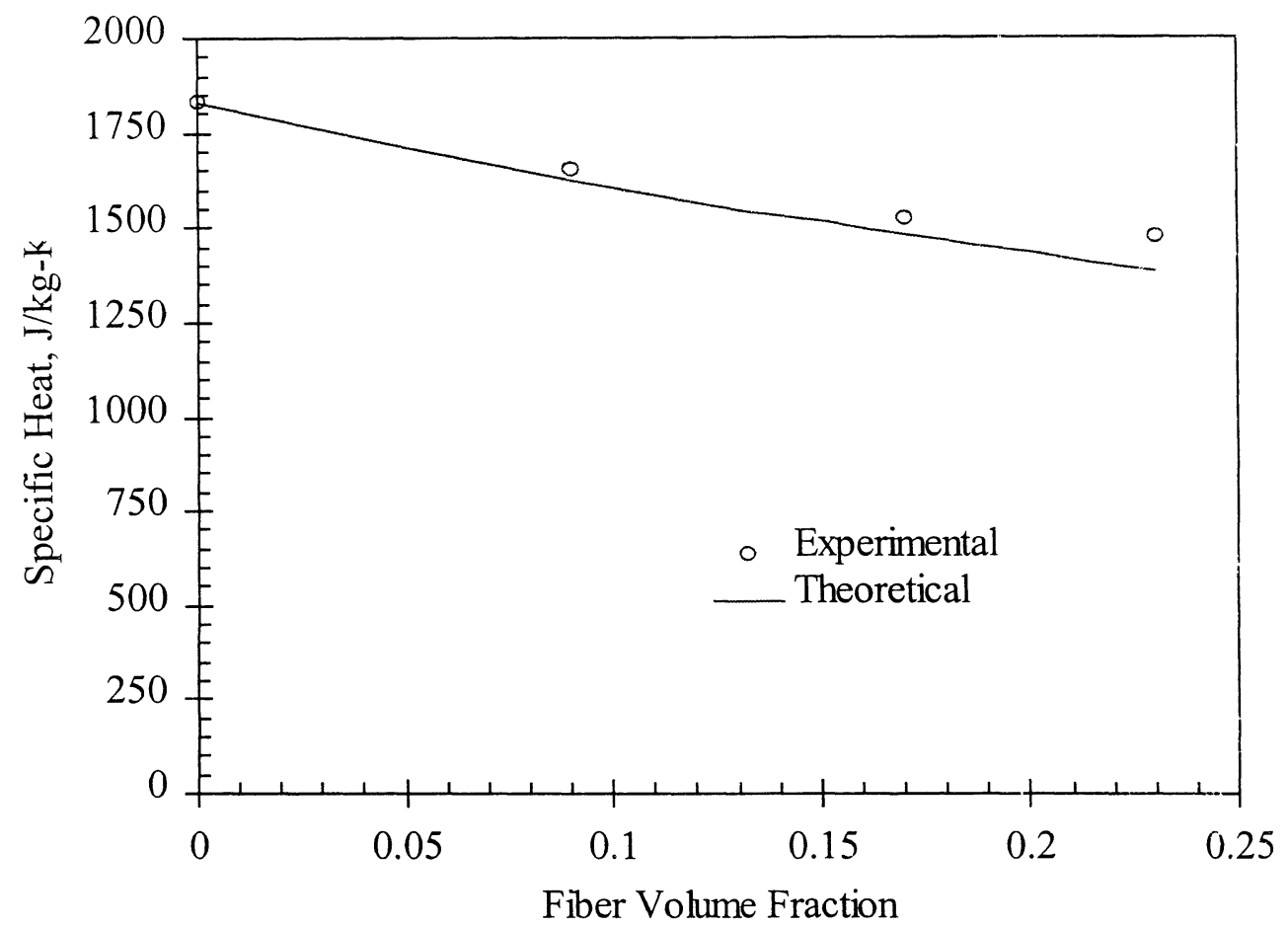

Fig. 6.2. Experimental heat capacity values of polypropylene reinforced with various amounts of Pyrograf III ${ }^{\mathrm{TM}}$ (PR-19-HT) VGCF. These values are compared with theoretical results calculated assuming the specific heat of the fiber equal to that of Pyrograf $I^{\mathrm{TM}}$.

The anisotropy of the thermal properties of VGCF is evident in Fig. 6.3, where the longitudinal diffusivity is seen to be much higher than the transverse diffusivity; although both quantities increased with fiber content. The pure polypropylene had a diffusivity of $0.0012 \mathrm{~cm}^{2} / \mathrm{s}$. The longitudinal diffusivity increases to $0.035 \mathrm{~cm}^{2} / \mathrm{s}$ with $23 \%$ fiber volume fraction, whereas, the transverse diffusivity increases to $0.014 \mathrm{~cm}^{2} / \mathrm{s}$. 


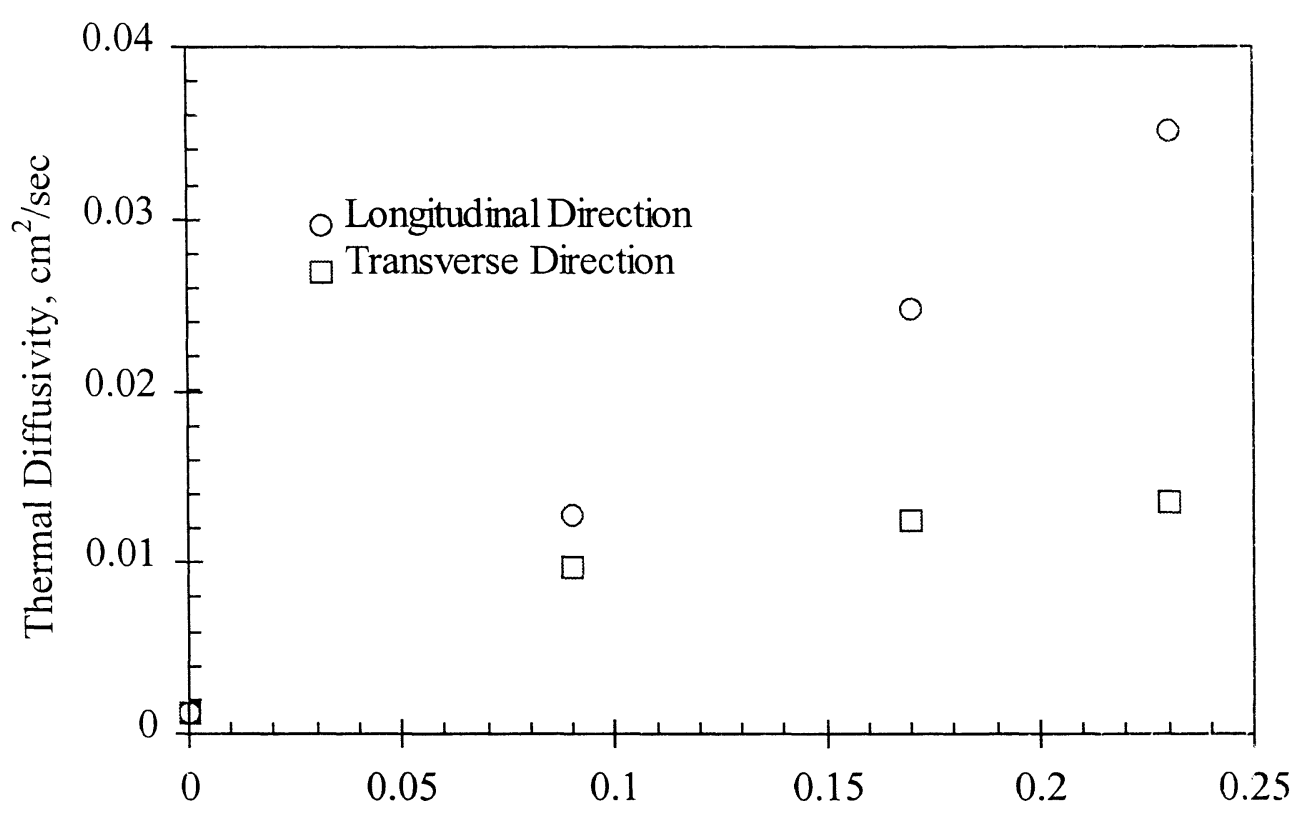

Fiber Volume Fraction

Fig. 6.3. Experimental thermal diffusivity results in the longitudinal and transverse directions for the polypropylene composites at various fiber volume fractions.

The experimental thermal conductivity results can be compared with theory and used to evaluate the axial and transverse thermal conductivity of the PR-19-HT fiber. There are numerous theoretical and empirical correlations for predicting the thermal conductivity of composite systems found in the literature. Each model works well for its own particular set of specific parameters, but no one method accurately predicts the thermal conductivity of all types of composite materials. It is particularly difficult to accurately apply existing models to VGCF composites due to the anisotropic nature of the fiber. The simple rule-of-mixtures (Callister Jr., 1994) or Cox's model (Gibson, 1994) used by other investigators (Patton et al., 1999; Dasch et al., 1993) for determining the thermal conductivity of VGCF composites tend to greatly over-predict the 
experimental data. This was also true for the present study. However, a semi-theoretical model developed by Lewis and Nielsen (Lewis and Nielsen, 1970; Nielsen, 1973; Nielsen, 1974) has been shown to accurately predict the thermal conductivity of polymer matrix composites reinforced with discontinuous fibers (Progelhof et al., 1976; Hoffman et al., 1989). This model also predicts the longitudinal and transverse thermal conductivity for uniaxially oriented fibers in the composite. Therefore, this model was used to predict the thermal conductivity of the VGCF composites produced for this study. The Lewis and Nielsen semi-theoretical model is a modified version of the Halpin-Tsai equation (Ashton et al., 1969), which includes the effects of the orientation and geometric shape of the filler particles. It also takes into account the type of packing for a two-phase system and the direction of the heat flow. The Lewis and Nielsen semitheoretical model for predicting the thermal conductivity $(k)$ of a two-phase system is given as

$$
k=k_{m}\left[\frac{1+A B V_{f}}{1-B V_{f} \psi}\right]
$$

where

$$
\begin{gathered}
B=\frac{\frac{k_{f}}{k_{m}}-1}{\frac{k_{f}}{k_{m}}+A} \\
\psi=1+\left(\frac{1-\phi}{\phi^{2}}\right) V_{f}
\end{gathered}
$$


In these expressions, $k_{f}$ and $k_{m}$ represent the thermal conductivity of the fiber reinforcement and the matrix material, respectively. The maximum packing fraction $(\phi)$ is set equal to 0.82 , which represents rod shaped particles packed in a uniaxial random configuration (characteristic of VGCF). The constant $A$ relates the shape and orientation of the fiber reinforcement and the direction of the heat flow. For uniaxially oriented fibers with the heat flow parallel to the fibers, $A=2 l / d$, and for heat flow perpendicular to the fibers, $A=0.5$. As described in Section 3.4, the average fiber length $(l)$ was found to be approximately 10.4 microns.

In Fig. 6.4 the experimental thermal conductivity in the longitudinal direction is compared with the theoretical model for uniaxially oriented fibers of different thermal conductivities. It can be observed that the theoretical curves for high values of fiber thermal conductivity tend to converge to a limiting value for each fiber volume fraction. Therefore, the curves for $k_{f}=200 \mathrm{~W} / \mathrm{m}-\mathrm{K}$ and $k_{f}=2000 \mathrm{~W} / \mathrm{m}-\mathrm{K}$ are within $10 \%$ of each other. The experimental values appear to indicate a $k_{f}$ value in the range of $200 \mathrm{~W} / \mathrm{m}-\mathrm{K}$ to $1000 \mathrm{~W} / \mathrm{m}-\mathrm{K}$. This is significantly less than the expected axial value of thermal conductivity $(2000 \mathrm{~W} / \mathrm{m}-\mathrm{K})$. However, from Chapter 4 , it should be noted that the fibers are not perfectly aligned along the sample axis (the composite cubes reinforced with $9 \%$, $17 \%$, and $23 \%$ fiber volume fraction had the fibers aligned within $\pm 36.5^{\circ}, \pm 38.7^{\circ}$, and $\pm 50.1^{\circ}$ along the preferred axis of the composite samples, respectively). Therefore, a reduction in the experimental value is expected. For the same reason the experimental transverse thermal conductivity results are expected to be higher than what is predicted by theory. This is confirmed in Fig. 6.5. It should be noted that the theoretical transverse 
thermal conductivity given by Eq. (6.8) predicts a conductivity result that is relatively insensitive to the value of $k_{f}$. Consequently the theoretical curve for $k_{f}=20 \mathrm{~W} / \mathrm{m}-\mathrm{K}$ is virtually the same as for $k_{f}=2000 \mathrm{~W} / \mathrm{m}-\mathrm{K}$. Therefore, only one theoretical curve has been shown for transverse thermal conductivity.

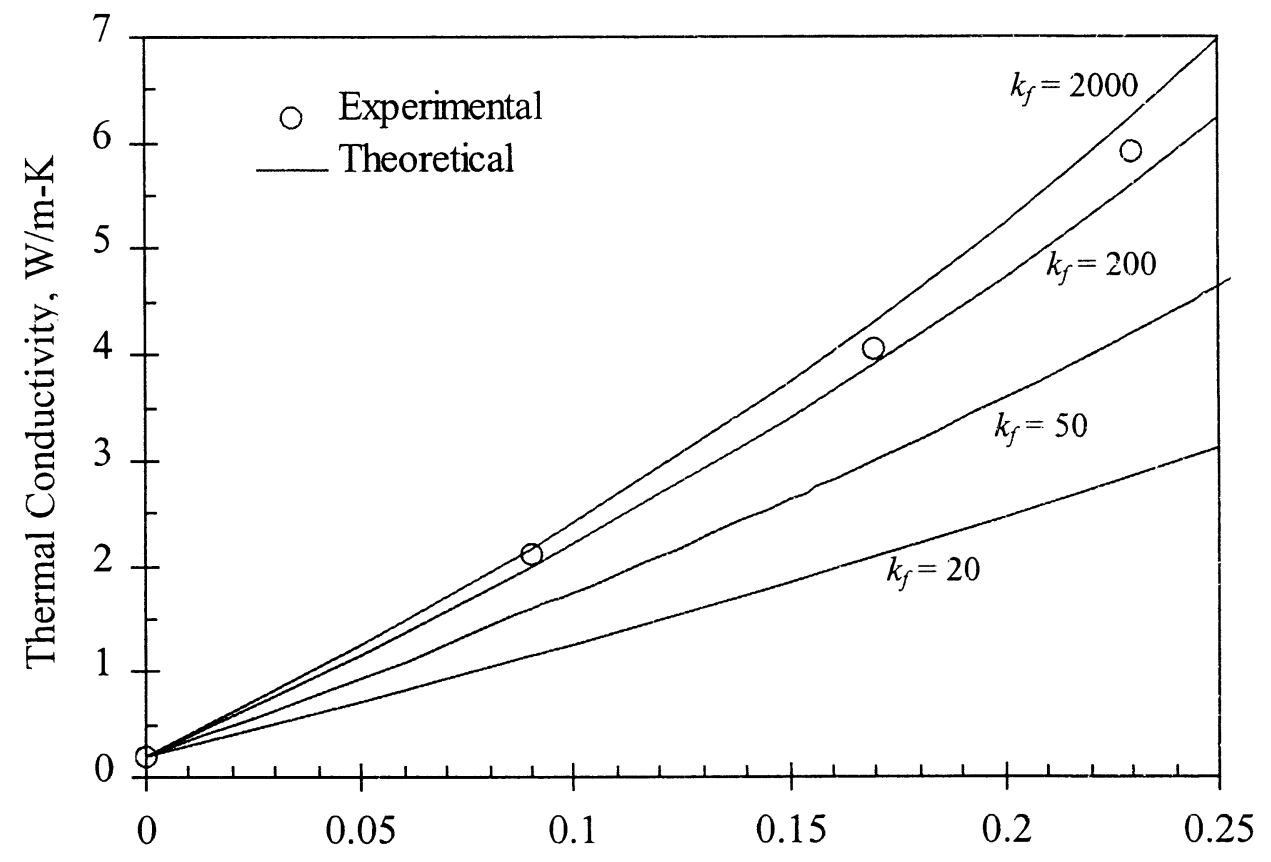

Fiber Volume Fraction

Fig. 6.4. Experimental thermal conductivity in the longitudinal direction compared with the semi-theoretical Lewis and Nielsen model for uniaxially oriented fibers of different thermal conductivities $\left(k_{f}\right.$ in $\left.\mathrm{W} / \mathrm{m}-\mathrm{K}\right)$. 


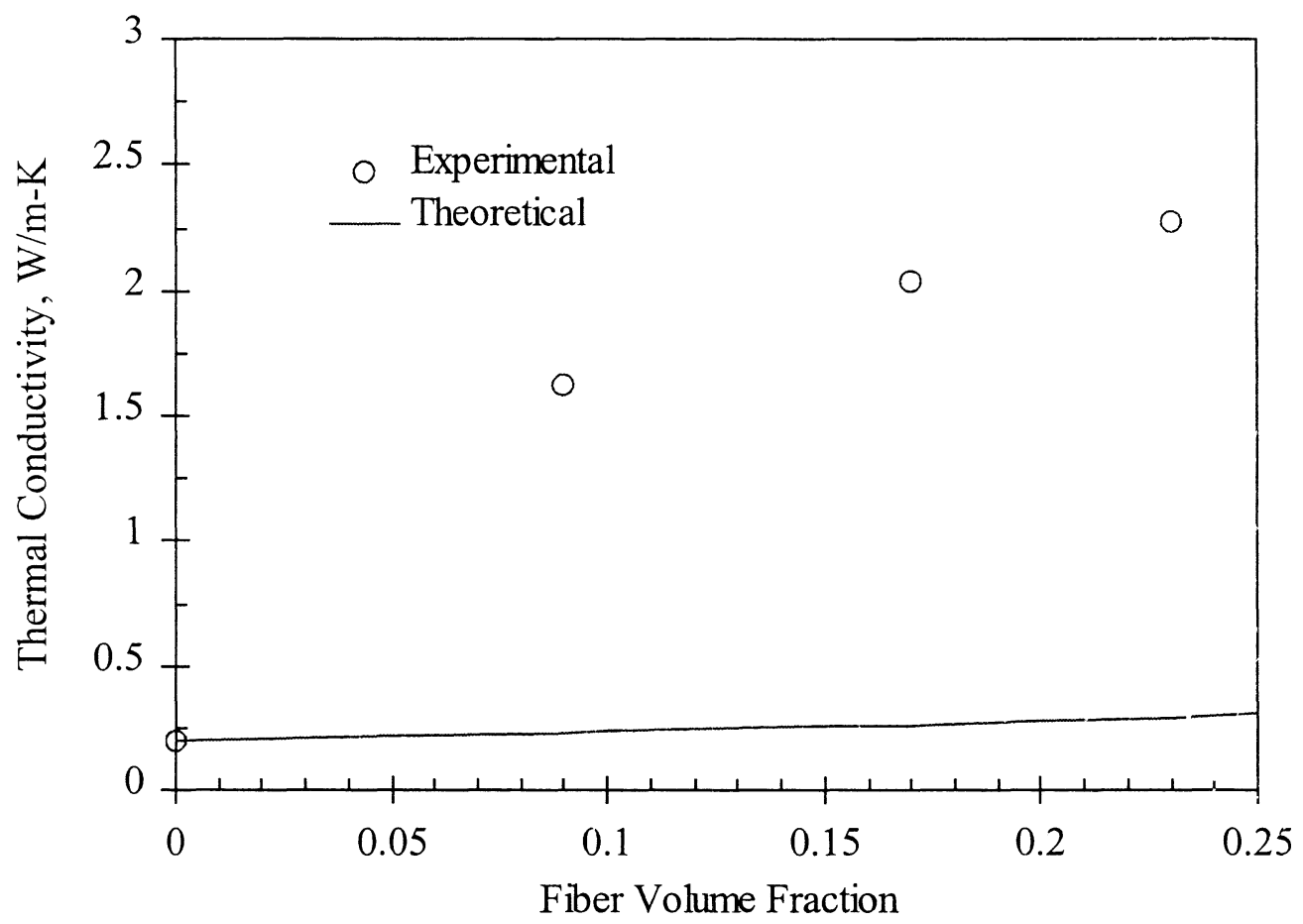

Fig. 6.5. Experimental thermal conductivity in the transverse direction compared with the semi-theoretical Lewis and Nielsen model for uniaxially oriented fibers of different thermal conductivities $\left(k_{f}=20 \mathrm{~W} / \mathrm{m}-\mathrm{K}\right)$.

The thermal conductivity results are also compared in Fig. 6.6 with VGCF composites found from other studies. It can be seen that the thermal conductivity values for the composite samples for this study are considerably higher than those obtained by Dasch et al. (1993) and Patton et al. (1999). The difference is due to the fact that the VGCF used in the current study has been heat-treated and are aligned within the composite materials to improve the conductivity. 


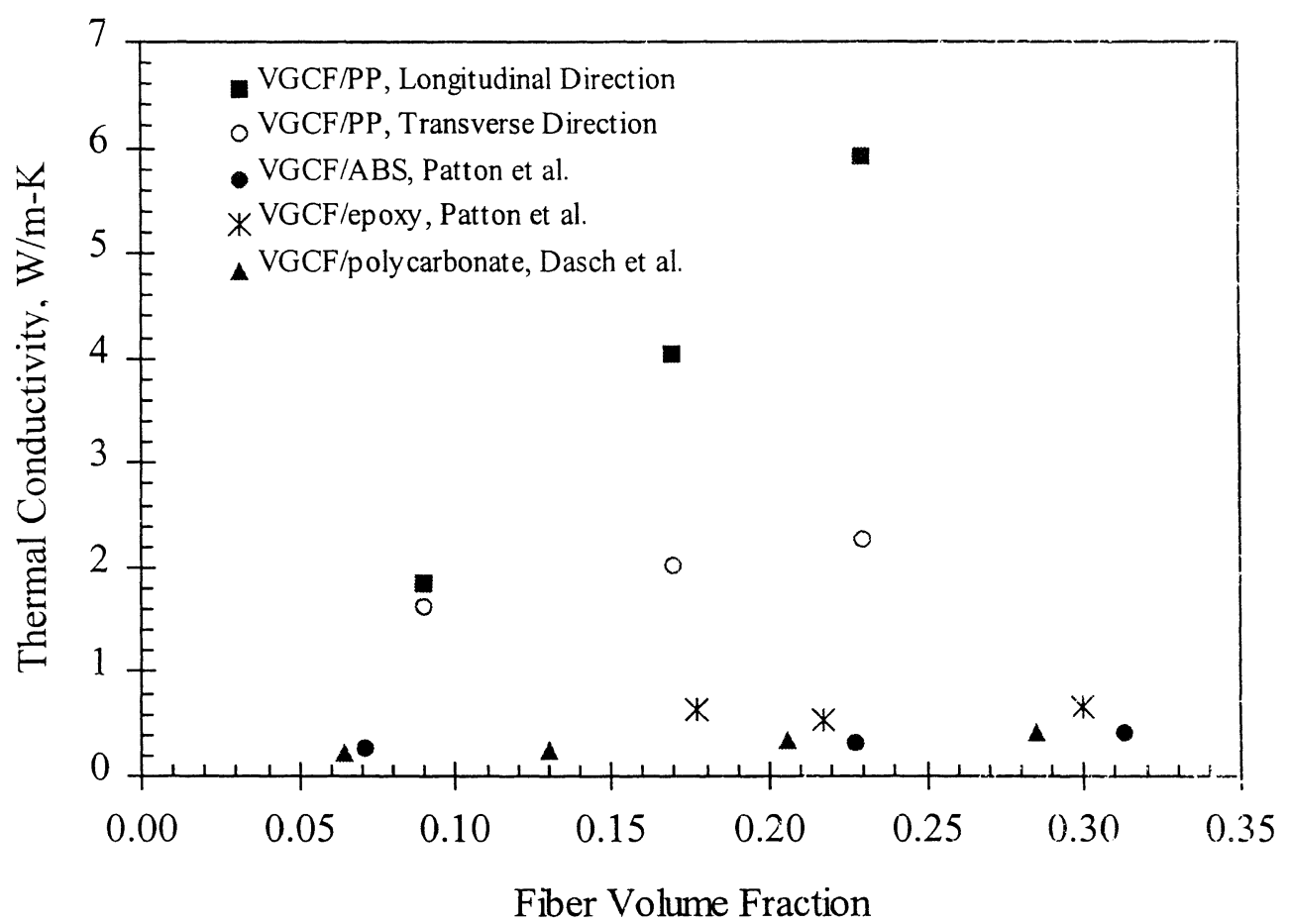

Fig. 6.6. Experimental thermal conductivity values of aligned, heat-treated fiber composites compared with results from other studies.

\subsection{Electrical Resistivity}

Electrical conductivity measurements were made on both the individual composite strands before the molding process and the molded cube samples. The resistivity of the uniform diameter $(\approx 2 \mathrm{~mm})$ composite strands was tested using a standard four-point testing apparatus. The control unit was adjusted to apply 15 volts and the current limit was set at 1.5 amps. The voltage drop $(V)$ and current $(i)$ of the test samples were measured between the two probes and the resistance was found using 


$$
R=\frac{V}{i}
$$

and the resistivity $(\lambda)$ of the composites was calculated by substituting into

$$
\lambda=R \frac{L}{A}
$$

In this equation, $L$ is the distance between the two measuring probes and $R$ is the resistance measured between them; $A$ is the cross sectional area of the samples.

To analyze the electrical conductivity of the cube specimens, they were first machined square. This was necessary due to the shrinkage and distortion of the cube during the cooling process. This also eliminates any possible insulating polymer layer, which may have formed during the molding process. As with the thermal conductivity measurements, the electrical resistivity in both the longitudinal $\left(\lambda_{L}\right)$ and transverse $\left(\lambda_{T}\right)$ directions of the cubes were tested. The analysis was performed by applying a uniform layer of Pelco Conductive Silver 415 (supplied by Ted Pella, Inc.) to the opposing faces of the cube along the test direction. Electrical leads were also bonded to the surfaces using the conductive silver paint. This eliminates contact resistance of the probes and allows for a basic two-point measurement to be performed using a digital multimeter. The resistance $(R)$ found using this method could then be substituted into Eq. $(6.12)$ to find the electrical resistivity of the composite samples.

Measurements were also performed in a similar manner for determining the resistivity of the individual sheets used to make the cubes. It can be seen from Table 6.2 that the electrical resistivity increases from the strand to the sheet and to the cube, and 
decreases with fiber volume fraction. This is due to the additional misalignment of fibers (Table 4.1) that occurs during the molding procedures.

Table 6.2. Electrical resistivity of PR-19-HT Pyrograf III ${ }^{\mathrm{MM}}$ fiber reinforced polypropylene at various fiber volume fractions.

\begin{tabular}{cccc}
\hline & \multicolumn{3}{c}{ Fiber Volume Percent } \\
\cline { 2 - 4 } Electrical Resistivity $($ Ohms-cm) & $9 \%$ & $17 \%$ & $23 \%$ \\
\hline $\begin{array}{c}\text { Extruded Strands }(\lambda) \\
\text { Molded Sheets }\end{array}$ & 3.3 & 0.234 & 0.105 \\
$\quad$ Longitudinal $\left(\lambda_{L}\right)$ & 3.6 & 0.26 & 0.17 \\
$\quad$ Transverse $\left(\lambda_{T}\right)$ & 5.62 & 1.35 & 0.98 \\
Molded Cubes & & & \\
L.ongitudinal $\left(\lambda_{L}\right)$ & 12.63 & 1.66 & 0.567 \\
Transverse $\left(\lambda_{T}\right)$ & 23.3 & 3.21 & 2.31 \\
\hline
\end{tabular}

By graphing the results (Figure 6.7), it is apparent that the electrical conductivity does not follow the rule-of-mixtures type of dependence as of the other transport properties. This occurs because three distinct processes control the electrical transport in a conductor-filled polymer system. Percolation is used to explain the macroscopic conduction in a disordered medium. Quantum mechanical tunneling describes the conduction between the adjacent fibers at the microscopic level. And thermal expansion is used to deduce the constituent volume densities and the microscopic turnel lengths (Sherman et al., 1983). Therefore, the exponential dependence on fiber volume fraction 
shown in Fig. 6.7 is indicative of these processes, where the resistivity of the pure polypropylene is approximately $10^{16} \mathrm{Ohms}-\mathrm{cm}$.

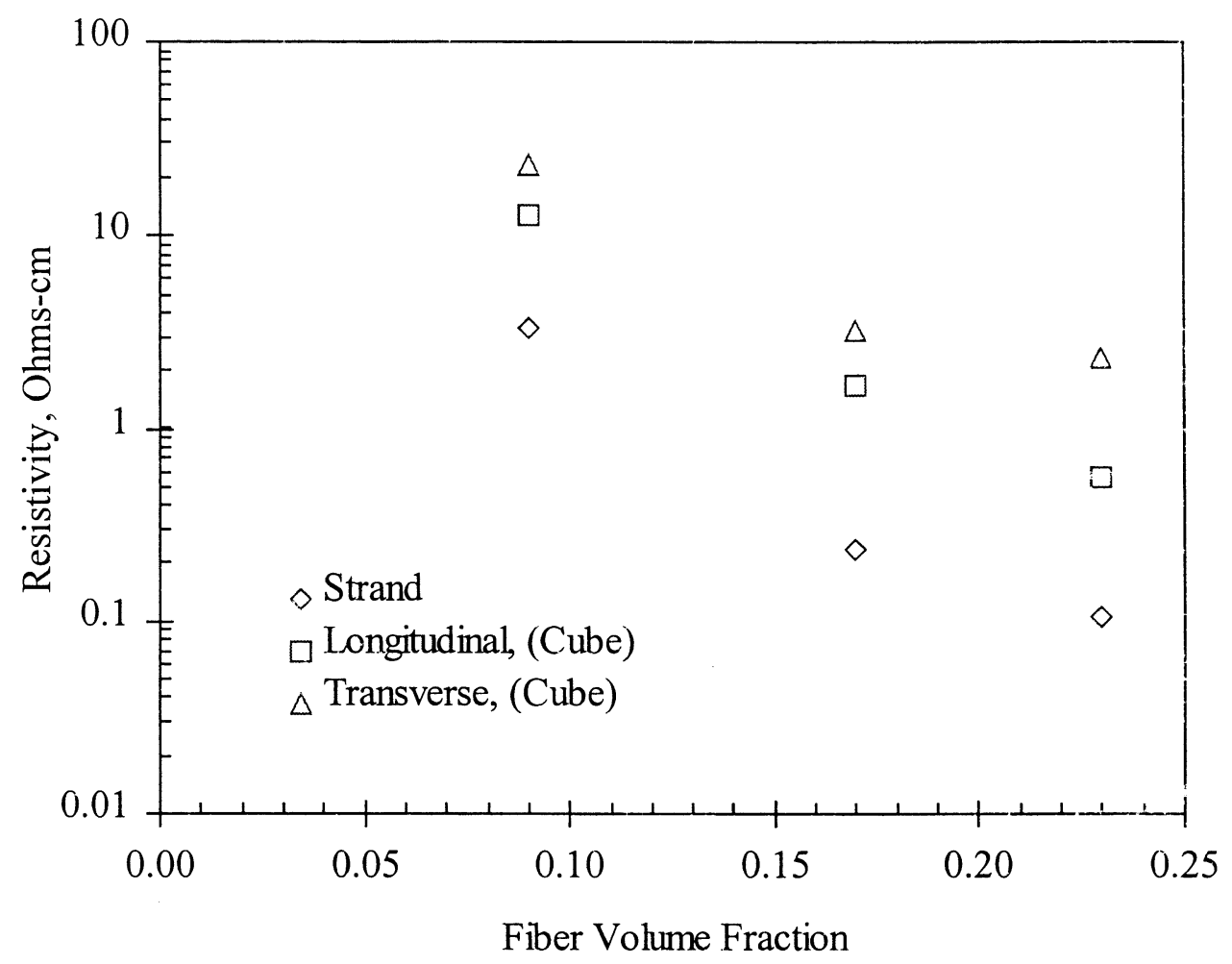

Fig. 6.7. Electrical resistivity of the extruded composite strands and the molded cube samples with various fiber volume fractions. 


\section{CHAPTER 7}

\section{CONCLUSIONS AND RECOMMENDATIONS FOR FUTURE STUDIES}

\subsection{Summary}

The work presented in this dissertation evaluated the extrusion process and properties of polypropylene composites reinforced with nanometer-size vapor grown carbon fiber (Pyrograf III ${ }^{\mathrm{TM}}$ ). The discontinuous Pyrograf III ${ }^{\mathrm{TM}}$ fiber (from Applied Sciences, Inc.) is microscopic in size and is produced from a floating-cataly'st in a continuous-flow reactor. This one-stage technique produces fibers at a much ingher rate and at lower costs. Thus, the potential low production costs of the Pyrograf IIIM vapor grown carbon fibers (VGCF) offers an opportunity for carbon fiber composites to expand into numerous new markets.

Understanding the extrusion process of VGCF reinforced composites is critical in order to process the composite into new products. The effect of extrusion temperature and screw speed on the process parameters (extrusion pressure, flow rate) and composite characteristics (tensile strength, modulus, and void content) were investigated. It was found that the extrusion process is strongly dependent on the fiber content of the composite. The addition of VGCF required an increase in the extrusion pressure. This seems to indicate that the viscosity of polypropylene is raised by addition of $\mathrm{VGCF}$. however, this needs to be verified by rheological measurements. As expected, the flow rate increased with an increase in extrusion temperature, whereas the extrusion pressure decreased. The tensile strength and modulus for the composite samples varied with 
extrusion temperature and screw speed; this variation was much smaller (less than 20\%) than that due to change in fiber volume fraction. The void content decreases with both the extrusion pressure and extrusion temperature, and increases with fiber fraction.

A method was also introduced for successfully aligning the nanometer-size VGCF suspended in a polymer flow during extrusion to produce an improved thermoplastic composite with preferred properties along a given direction. A converging-annular die configuration was used to produce flow-induced alignment of the VGCF in a uniform diameter composite strand. It was shown that degree of fiber alignment along the preferred direction could be improved by increasing the residence time of the composite flow through the die channel. This was verified by attaching an extended annular nozzle to the die. The degree of fiber alignment was quantified by using $x$-ray diffraction. It was determined that the amount of orientation along the preferred axis decreases as the fiber content increases.

An increase in fiber alignment along a preferred direction also increases the properties of the composite materials in that direction. To demonstrate this, tensile test samples were machined from sheets fabricated by molding the extruded composite strands. To evaluate the thermal and electrical conductivity, the composite sheets were used as prepegs and remolded to produce a laminated anisotropic composite cube. X-ray diffraction showed that the deformation of the composite strands when consolidated by this molding procedure increases the degree of fiber misalignment.

Test results showed that mechanical properties of the polypropylene were consistently improved with the increase in fiber volume fraction. Also, the tensile 
strength improved with greater fiber orientation, however, increasing fiber alignment had little affect on the modulus. When compared with theoretical models, the strength was observed to be intermediate between a uniaxial (1-D) model and a randomly oriented 2-D model. The modulus value did not increase beyond the 2-D model and the increase at high fiber loading is intermediate between the 2-D and 3-D models. It was shown that shearing forces produced by the extruder screws in the high viscosity fiber/polymer mixtures degraded the length of the fibers. The tensile strength also decreased at higher screw speeds, which indicated that fiber length may be reduced due to higher shear. However, due to the entanglement of the fibers, tests on fiber length were not conclusive enough to accurately quantify the change in fiber length at different processing conditions.

An experimental and theoretical approach was described for the determination of the strength of partially aligned discontinuous fiber reinforced composites. The fiber alignment information was obtained as a Gaussian or normal distribution function by using a x-ray diffraction technique. The distribution function was then used in the composite strength equation to calculate the theoretical strength. The theoretical calculations made using this model corresponded closely to the experimental results of the partially aligned composite evaluated in this study. It was also observed that the composite strength is sensitive to variation in fiber length when the average fiber length is less than the critical fiber length. At higher fiber volume fractions the composite strength was much lower than predicted by theory, which is attributed to incomplete wetting and infiltration of the VGCF. 
Finally, the thermal and electrical properties of the polymer composites reinforced with aligned heat-treated Pyrograf III $^{\mathrm{TM}}$ fibers were investigated. The density, specific heat, and thermal diffusivity of the composite samples were experimentally determined and used to calculate the thermal conductivity in both the longitudinal and transverse directions. The density of the composite samples increased with fiber content, whereas the specific heat decreased. This was expected since the density of the fiber is much greater than the polypropylene, while the heat capacity is less. The thermal diffusivity and the corresponding thermal conductivity increased with fiber content, and were much higher in the longitudinal direction compared with the transverse direction due to axial alignment of the fibers. When compared with the semi-theoretical Lewis and Nielsen model, the experimental values were over-predicted in the longitudinal direction and much greater than predicted in the transverse direction. This occurs because the fibers are not perfectly aligned along the axis of the test samples.

The electrical resistivity of the aligned VGCF composite samples decreased with fiber content, and was much less in the longitudinal direction, opposed to the transverse direction. The electrical conductivity has an exponential dependence on fiber volume fraction and does not follow the rule-of-mixtures type of dependence as of the other transport properties. This is indicative of the electrical transport in conductor-filled polymer systems, which are governed by percolation, quantum mechanical tunneling between the fibers, and thermal expansion. 


\subsection{Recommendations for Future Studies}

There are several issues that need to be addressed in order to improve the understanding of the extrusion process and modeling of VGCF composites. For example, in order to design a die that produces a maximum amount of fiber alignment it is necessary to model the flow properties of VGCF reinforced polymer composites. The flow behavior of VGCF suspensions will prove to be a challenging problem of fundamental interest. Critical issues such as the effect of fiber-fiber interactions, fiberwall interactions, geometry of the fiber, the type of flow field, and the rheology of the molten fiber/polymer mixture must be understood in order to predict and model the flow behavior of VGCF suspended in a polymer matrix. Further experimentation of the $\mathrm{VGCF} /$ polymer blends must be performed to determine the dependence of viscosity on the fiber content, shear rate, and temperature which is necessary in order to model the flow of the composite mixtures.

Once the rheological properties of the VGCF suspensions are known, it would then be possible to use a commercial software package to model and design an extrusion die which maximizes the flow-induced alignment of the fiber. In addition to modeling simpler flows that is experienced in the simple dies used for this study, more complex designs could be investigated. A die could be designed to generate fiber alignment when extruding more complex shapes such as a ribbon or triangular cross-section.

The $\mathrm{x}$-ray diffraction technique used in Chapter 5 to predict the strength of partially aligned fiber composites could also be utilized for predicting the thermal conductivity of partially aligned isotropic fiber composites. This would prove to be a 
useful tool since virtually all manufacturing processes produce composites that contain fibers partially aligned along the flow direction. Such a model could then be extended to predict the thermal conductivity of composites reinforced with an anisotropic fiber such as VGCF.

Finally, this study was conducted using only polypropylene as the matrix material; however, the performance of various other matrices such as nylon, polyethylene, or polycarbonate should be evaluated in future experiments. 


\section{REFERENCES}

Agarwal, B. D., and Broutman, L. J., 1990, Analysis and Performance of Fiber Composites, John Wiley \& Sons, Inc., New York, NY.

Alexander, L. E., 1969, X-Ray Diffraction Methods in Polymer Science, WileyInterscience, John Wiley \& Sons, Inc., New York. Chapter 4.

Ali, Z., Alam, M. K., Storch, A., 1995, "Thermal Properties of Advanced Fiber Reinforced Composite Materials," ASME PVP Proc., Vol. 313, pp. 179-188.

Applied Sciences, Inc., 1999, "Product data sheet for Pyrograf III type PR-21-AG."

Ashton, J. E., Halpin, J. C., and Petit, P. H., 1969, "Primer on Composite Materials Analysis,” Technomic Pub. Co., Stamford, CT, Chap. 2.

ASI, 1999, "Low Cost Carbon Fibers for Industrial Applications," http://www.apsci.com/pub1.html.

ASTM D 792, 1993, "Standard Test Methods for Density and Specific Gravity (Relative Density) of Plastics by Displacement," Annual Book of ASTM Standards, Vol. 8.01, pp. 293-296.

Azzi, V. D., and Tsai, S. W., 1965, Proceedings of the Society for Experimental Stress Analysis, XXII(2), pp. 283.

Baxter, W. J., 1992, "The Strength of Metal Matrix Composites Reinforced with Randomly Oriented Discontinuous Fibers," Metallurgical Transactions, Vol. 23A, pp. 3045-3053. 
Bunsell, A. R., 1988, Fibre Reinforcements for Composite Materials, pp. 73-145, Elsevier Science Publishing Co., New York, NY.

Caldeira, G., Mala, J. M., Carneiro, O. S., Covas, J. A., and Bernardo, C. A., 1998, "Production and Characterization of Innovative Carbon Fiber-Polycarbonate Composites," Polymer Composites, Vol. 19, No. 2, pp.147-151.

Callister Jr., W. D., 1994, Materials Science and Engineering: an Introduction. $3^{\text {rd }}$ ed., New York: Wiley, pp. 528

Carneiro, O. S., Covas, J. A., Bernardo, C. A., Caldeira, G., Van Hattum, F. W., Ting J.. M., Alig, R. L., and Lake, M. L., 1998, "Production and Assessment of Polycarbonate Composites Reinforced with Vapour-Grown Carbon Fibres," Composite Science and Technology, Vol. 58, pp. 401-407.

Chen, P. E., 1971, "Strength Properties of Discontinuous Fiber Composites," Polymer Engineering and Science, Vol. 11, No. 1, pp. 51-55.

Chiu, W. Y., Lu, H. C., and Chang, C. L., 1991, "Relationship Between Extrusion Condition and Mechanical Properties of FRPP," J. Appl. Poly. Sci., Vol 43, pp. 13351341.

Ciminelli, David L., Kearns, Kristen M., Ragland, William R., 1996, "Vapor Grown Carbon Fiber (VGCF) Composites," Proceeding of the $41^{\text {st }}$ International SAMPE Symposium and Exhibition, Anaheim, CA, Vol. 41, No. 1, pp. 495-501.

Cowan, J., 1963, "Pulse Method of Measuring Thermal Diffusivity at High Temperatures," Journal of Applied Physics, Vol. 34, pp. 926. 
Cox, H. L., 1952, "The Elasticity and Strength of Paper and Other Fibrous Materials," British Journal of Applied Physics, Vol. 3, pp. 72-79.

Daimaru, A., 1990, "Method of Controlling the Orientation of Short Fibers in a Short Fiber-Reinforced Article." U.S. Patent No. 4,938,905.

Dasch, C. J., and Alig, R. L., 1995, "Composite of Small Carbon Fibers and Thermoplastics and Method for Making Same." U.S. Patent No. 5,433,906.

Dasch, C. J., Baxter, W. J., and Tibbetts, G. G., 1993, “Thermoplastic Composites using Nanometer-Size Vapor-Grown Carbon Fibers," $21^{\text {st }}$ Biennial Conference on Carbon, pp. 82.

Dospisil, D., Kubat, J., Plesek, M., and Saha, P., 1994, "Extrusion of Short Fibre Reinforced PP," Intern. Polym. Processing, Vol. 9, pp. 303.

Dutta, D., Husband, M., Ciminelli, D., and Hager, J. W., 1995, "Processing of VGCFThermoplastic Composites with Aligned Extruded Tapes," The $22^{\text {st }}$ Biennial Conference on Carbon, pp. 292.

El-Sobky, H., 1999, “Extrusion Die.” U.S. Patent No. 5,888,555.

Endo, M. and Komaki, K., 1983, "Formation of Vapor-Grown Carbon Fibers by Seeding Method of Metal Ultra-Fine Particles," Ext. Abst. 16th Biennial Conf. Carbon, San Diego, CA, pp. 523.

Gibson, R. F., 1994, Principles of Composite Material Mechanics, McGraw-Hill, Inc., New York, NY, Chap. 6.

Glasgow, J., Applied Sciences, Inc., Cedarville, OH, 2000, "Private communications." 
Goettler, L. A., and Lambright, J., 1977, "Process for Controlling Orientation of Discontinuous Fiber in a Fiber-Reinforced Product Formed by Extrusion." U.S. Patent No. 4,056,591.

Hoffman, R. R., Tye, R. P., and Chervenak, J. G., 1989, "Investigation into Thermal Conductivity of Composite Materials for Electronics Packaging," Advances in Thermoplastic Matrix Composite Materials, ASTM STP 1044, G. M. Newaz, ed., American Society for Testing and Materials, Philadelphia, pp. 146-153.

Hughes, T. V., and Chambers, C. R., 1889, U.S. Patent No. 405,480.

Jarrin, J., Vinciguerra, E., Ausias, G., Vincent, M., and Dawans, F., 1994, "Extrusion of Tubes of Fiber-Reinforced Thermoplastics.” U.S. Patent No. 5,307,843.

Katsuki, H., Matsunaga, K., Egashira, M., and Kawasumi, S., 1981, "Formation of Carbon Fibers from Naphthalene on Some Sulfur Containing Substrates," Carbon, Vol. 19, pp. 148.

Kelly, A., and Tyson, W. R., 1965, “Tensile Properties of Fibre Reinforced Metals: Copper/Tungsten and Copper/Molybdenum," Journal of the Mechanics and Physics of Solids, Vol. 13, pp. 329-350.

Knoblach, G. M., 1991, "Electric Alignment of Fibers for the Manufacture of Composite Materials.” U.S. Patent No. 5,057,253.

Koyama, T., 1972, "Formation of Carbon Fibers from Benzene," Carbon, Vol. 10, pp. 757.

Koyama, T., and Endo, M., 1973, "Structure and Growth Processes of Vapor-Grown Carbon Fibers," O. Buturi, Vol. 42, pp. 690. 
Lake, M., Ting, J. M., and Alig, R., 1992, "Vapor Grown Carbon Fibers: Properties and Applications," 8 ${ }^{\text {th }}$ Annual Conf. on Materials Technology, pp. 55-64, 1992.

Laun, H. M., 1984, “Orientation Effects and Rheology of Short Glass Fiber-Reinforced Thermoplastics," Colloid \& Polym. Sci., Vol. 262, pp. 257.

Lees, J. K., 1968, "A Study of the Tensile Strength of Short Fiber Reinforced Plastics," Polymer Engineering and Science, Vol. 8, No. 1, pp. 195-201.

Lewis, T., and Nielsen, L., 1970, "Dynamic Mechanical Properties of Particulate-Filled Polymers," J. Appl. Polym. Sci., Vol. 14, pp. 1449.

Masuda, T., Mukai, S. R., and Hashimoto, K., 1993, "The Liquid Pulse Injection Technique: A New Method to Obtain Long Vapor Grown Carbon fibers at High Growth Rates", Carbon, Vol. 31, No. 5, pp. 783-787.

McPhee, D. J., and Gray, H. W., 1980, "Extruder Head for Making Elastomer-Fiber Composite Hose." U.S. Patent No. 4,240,782.

Miani, M., 1995, "Two-Component Extrusion Head, Having a Spinneret with High Perforation Intensity." U.S. Patent No. 5,466,142.

Michel, R. H., 1991, "Extruder Process for Preparation of Carbon Fiber Reinforced Fluoropolymer Compositions." U.S. Patent No. 4,422,992.

Montell U.S.A., Inc., 1999, "Material data sheet for Pro-fax 6301 polypropylene homopolymer."

Morgan, R. G., 1989, "Directional Flow Bar Extruder." United States Patent No. $4,883,421$. 
Mortensen, A., Masur, L. J., Cornie, J. A., and Flemings, M. C., 1989, "Infiltration of Fibrous Preforms by a Pure Metal. Part 1. 'Theory," Metallurgical Transactions A, Vol. 20A, No. 11, pp. 2535-2547.

Nielsen, L., 1973, "Thermal Conductivity of Particulate-Filled Polymers," J. Appl. Polym. Sci., Vol. 17, pp. 3819.

Nielsen, L., 1974, "The Thermal and Electrical Conductivity of Two-Phase Systems," Ind. Eng. Chem. Fund., Vol. 13, pp. 17.

Nomura, S., and Chou, T. W., 1980, "Bounds of Effective Thermal Conductivity of Short-Fiber Composites," J. Comp. Mat., Vol. 14, pp.120-129.

Parker et al., 1961, "Flash Method of Determining Thermal Diffusivity, Heat Capacity, and Thermal Conductivity," Journal of Applied Physics, Vol. 32, pp. 1679.

Patton, R. D., Pittman Jr., C. U., Wang, L., and Hill, J. R., 1999, "Vapor Grown Carbon Fiber Composites with Epoxy and Poly(phenylene sulfide) Matrics," Composites: Part A, Vol. 30, pp. 1081-1091.

Prewo, K. M., and Kreider, K. G., 1972, “The Transverse Tensile Properties of Boron Fiber Reinforced Aluminum Matrix Composites," Metallurgical Transactions, Vol. 3, pp. 2201-2211.

Progelhof, R. C., Throne, J. L., and Ruetsch, R. R., 1976, "Methods for Predicting the Thermal Conductivity of Composite Systems: A Review," Polymer Engineering and Science, Vol. 16, No. 9, pp. 615-625. 
Ranganathan, S., and Advani, S., 1997, Flow-Induced Alignment in Composite Materials, Papathanasiou, T. D. and Guell, D. C., eds., Woodhead Publishing Ltd., Cambridge, England, pp. 43-45.

Roe R. J., and Krigbaum, W. R., 1964, "Description of Crystallite Orientation in Polycrystalline Materials Having Fiber Texture," J. Chem. Phys., Vol. 40, pp. 2608.

Ruland, W., 1968, "X-Ray Diffraction Studies on Carbon and Graphite," Chemistry and Physics of Carbon: A Series of Advances, Vol. 4, pp 1-84, Philip L. Walker Jr., Ed., Marcel Dekker, Inc., New York.

Sargent, M. M., 1995, “Apparatus for Compounding a Fiber Reinforced Thermoplastic Material and Forming Parts Therefrom." U.S. Patent No. 5,401,154.

Sherman, R. D., Middleman, L. M., and Jacobs, S. M., 1983, "Electron Transport Processes in Conductor-Filled Polymers," Polym. Eng. Sci., Vol. 23, No. 1, pp. 36-46. Tepic, S., 1992, "Method for Producing Oriented, Discontinuous Fiber Reinforced Composite Materials.” U.S. Patent No. 5,093,050.

Tibbetts, G. G., General Motors Research and Development Center, Warren, MI, 2000, "Private Communications."

Tibbetts, G. G., and Beetz Jr., C. P., 1997, "Mechanical Properties of Vapour-Grown Carbon Fibers," J. Physics D, Vol. 20, pp. 292.

Tibbetts, G. G., and Devour, M. G., 1986, "Regulation of pyrolysis methane concentration in the manufacture of graphite fibers." U.S. Patent No. 4,565,684. 
Tibbetts, G. G., Gorkiewicz, D. W., Alig, R. L., 1993, “A New Reactor for Growing Carbon Fibers from Liquid and Vapor Phase Hydrocarbons." Carbon, Vol. 31, pp. 809-814.

Tibbetts, G. G., Gorkiewicz, D. W., Hammond Jr., D. C., 1991, “Apparatus for Forming Carbon Fibers.” U.S. Patent No. 5,024,818.

Tibbetts, G. G., and McHugh, J. J., 1999, "Mechanical Properties of Vapor-Grown Carbon Fiber Composites with Thermoplastic Matrices." Journal of Materials Research, Vol. 14, No. 7, pp. 2871-2880.

Tsai, S. W., 1968, Fundamental Aspects of Fiber Reinforced Plastic Composites, edited by R. T. Schwartz and H. S. Schwartz, Wiley Interscience, New York, NY, pp. 3-11.

Van Hattum, F. W. J., Bernardo, C. A., Finegan, J. C., Tibbetts, G. G., Alig, R. L., and Lake, M. L., 1999, “A Study of the Thermomechanical Properties of Carbon FiberPolypropylene Composites," Polymer Composites, Vol. 20, No. 5, pp. 683-688.

Yamanashi, H., Ushijima, H., Katsumata, M., and Endo, M., 1993, “Properties of Epoxy Composite using Vapor-Grown Carbon Fibers (VGCF's) as an Advanced Adhesive Agent," The $21^{\text {st }}$ Biennial Conference on Carbon, pp. 88.

Zhang, C., Yi, X. S., Yui, H., Asai, S., and Sumita, M., 1998, "Morphology and Electrical Properties of Short Carbon Fiber-Filled Polymer Blends: High-Density Polyethylene/Poly(methyl methacrylate)," Journal of Applied Polymer Science, Voi. 69, pp. 1813-1819. 
KURIGER, REX, JERRALD. Ph.D. November 2000

Mechanical Engineering

Improved Thermoplastic Composite by Alignment of Vapor Growr Carbon Fiber (122 pp.)

Director of Dissertation: Dr. M. Khairul Alam

Vapor grown carbon fiber (VGCF) is a new and inexpensive carbon fiber produced by vapor deposition of hydrocarbons on metal catalysts. Unijke continuous conventional PAN or pitch-derived carbon fibers, VGCF is discontinuous with diameters of about 200 nanometers and lengths ranging from 10 to 200 microncters. The microscopic size and random entanglement of the fibers create several probiems when processing VGCF composites. It is particularly difficult to disperse the entangied ifibers in the matrix and orient them along a preferred axis to provide directional reinforcement. This work introduces a technique to produce an improved polynenc composite $b y$ alignment of vapor grown carbon nano-fibers in a polypropylene matrix.

A twin-screw extruder was used to shear mix and disperse the fibers in the polymer matrix. The composite mixtures were extruded through a converging-emmular die that generates flow-induced fiber alignment along the extrusion direction. The effect that the various extrusion conditions have on the bulk properties of the extiudate was investigated. It was found that the extrusion process is strongly dependent on the fiber content of the composite. The extrusion pressure increased and the flow rate decreased with fiber volume fraction. The tensile strength and modulus for the composite samples 